\title{
Nuclear Energy Advanced Modeling and Simulation (NEAMS) Waste Integrated Performance and Safety Codes (IPSC): FY10 Development and Integration
}

Geoff Freeze, J. Guadalupe Argüello, Julie Bouchard, Louise Criscenti, Thomas Dewers, H. Carter Edwards, David Sassani, Peter A. Schultz, and Yifeng Wang 
Issued by Sandia National Laboratories, operated for the United States Department of Energy by Sandia Corporation.

NOTICE: This report was prepared as an account of work sponsored by an agency of the United States Government. Neither the United States Government, nor any agency thereof, nor any of their employees, nor any of their contractors, subcontractors, or their employees, make any warranty, express or implied, or assume any legal liability or responsibility for the accuracy, completeness, or usefulness of any information, apparatus, product, or process disclosed, or represent that its use would not infringe privately owned rights. Reference herein to any specific commercial product, process, or service by trade name, trademark, manufacturer, or otherwise, does not necessarily constitute or imply its endorsement, recommendation, or favoring by the United States Government, any agency thereof, or any of their contractors or subcontractors. The views and opinions expressed herein do not necessarily state or reflect those of the United States Government, any agency thereof, or any of their contractors.

Printed in the United States of America. This report has been reproduced directly from the best available copy.

Available to DOE and DOE contractors from

U.S. Department of Energy

Office of Scientific and Technical Information

P.O. Box 62

Oak Ridge, TN 37831

Telephone: $\quad$ (865) 576-8401

Facsimile: $\quad$ (865) 576-5728

E-Mail: $\quad$ reports@adonis.osti.gov

Online ordering: http://www.osti.gov/bridge

Available to the public from

U.S. Department of Commerce

National Technical Information Service

5285 Port Royal Rd.

Springfield, VA 22161

Telephone: $\quad$ (800) 553-6847

Facsimile: $\quad$ (703) 605-6900

E-Mail: $\quad$ orders@ntis.fedworld.gov

Online order: $\quad$ http://www.ntis.gov/help/ordermethods.asp?loc=7-4-0\#online

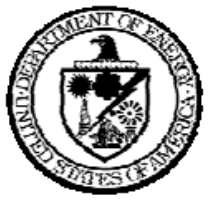


SAND2011-0845

Unlimited Release

February 2011

\title{
Nuclear Energy Advanced Modeling and Simulation (NEAMS) Waste Integrated Performance and Safety Codes (IPSC): FY10 Development and Integration
}

\author{
Geoff Freeze, J. Guadalupe Argüello, Julie Bouchard, Louise Criscenti, \\ Thomas Dewers, H. Carter Edwards, David Sassani, Peter A. Schultz, and Yifeng Wang
}

Sandia National Laboratories

P.O. Box 5800

Albuquerque, New Mexico 87185-MS1369

\begin{abstract}
This report describes the progress in fiscal year 2010 in developing the Waste Integrated Performance and Safety Codes (IPSC) in support of the U.S. Department of Energy (DOE) Office of Nuclear Energy Advanced Modeling and Simulation (NEAMS) Campaign. The goal of the Waste IPSC is to develop an integrated suite of computational modeling and simulation capabilities to quantitatively assess the long-term performance of waste forms in the engineered and geologic environments of a radioactive waste storage or disposal system. The Waste IPSC will provide this simulation capability (1) for a range of disposal concepts, waste form types, engineered repository designs, and geologic settings, (2) for a range of time scales and distances, (3) with appropriate consideration of the inherent uncertainties, and (4) in accordance with robust verification, validation, and software quality requirements.

Waste IPSC activities in fiscal year 2010 focused on specifying a challenge problem to demonstrate proof of concept, developing a verification and validation plan, and performing an initial gap analyses to identify candidate codes and tools to support the development and integration of the Waste IPSC. The current Waste IPSC strategy is to acquire and integrate the necessary Waste IPSC capabilities wherever feasible, and develop only those capabilities that cannot be acquired or suitably integrated, verified, or validated. This year-end progress report documents the FY10 status of acquisition, development, and integration of thermal-hydrologicchemical-mechanical (THCM) code capabilities, frameworks, and enabling tools and infrastructure.
\end{abstract}




\section{ACKNOWLEDGEMENTS}

This work was supported by the U.S. Department of Energy, Office of Nuclear Energy, Fuel Cycle Research and Development Program, Advanced Modeling and Simulation Campaign.

Charles Bryan supported the THCM code capability gap analysis by compiling the list of potentially relevant codes in Appendix A. Helpful review comments were provided by Pat Brady. 


\section{CONTENTS}

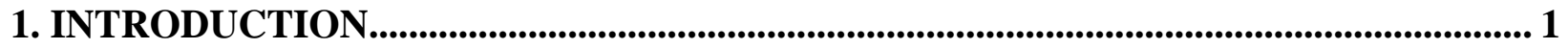

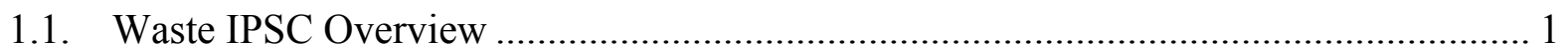

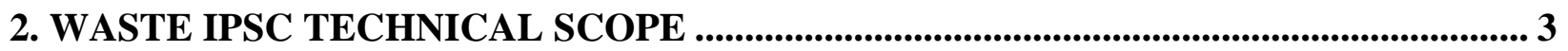

3. CHARACTERIZATION OF SUB-CONTINUUM PROCESSES....................................... 9

3.1. Overview of Glass Waste Form Dissolution …………............................................. 10

3.1.1. Repository Settings - Why and When Glass Waste Form Dissolution is

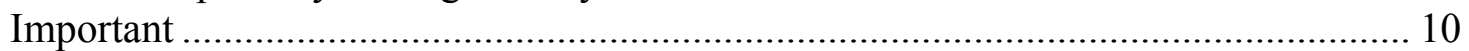

3.1.2. Context of Glass Degradation........................................................................ 11

3.1.3. Current Understanding and Gap Identification............................................... 15

3.2. Continuum-Scale Rate Models for Glass Dissolution .................................................... 20

3.2.1. Overview of Kinetic Dissolution Rate Expressions......................................... 20

3.2.2. Quantification of Rate Law Parameters........................................................ 23

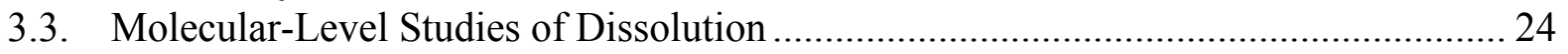

3.3.1. Determination of Reaction Mechanisms ..................................................... 24

3.3.2. Quantum Mechanics Cluster Calculations.................................................. 25

3.3.3. Classical Molecular Dynamics (MD) Models .................................................. 31

3.3.4. Kinetic Monte Carlo (MC) Models for Dissolution ......................................... 33

3.3.5. Stochastic Monte Carlo (MC) Models for Dissolution.................................... 33

3.3.6. Modeling Mesoscale Effects on Glass...................................................... 42

3.3.7. Experimental Validation of Molecular Models .............................................. 44

3.3.8. Summary of Gaps in Upscaling Dissolution Processes ................................ 46

3.4. Verification, Validation, and Uncertainty Quantification............................................. 47

3.4.1. Practices for Sub-Continuum-Scale Modeling .............................................. 47

3.4.2. Upscaling with Propagating Uncertainties..................................................... 48

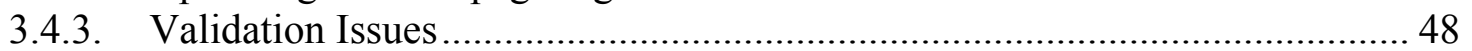

3.4.4. Evidence Management................................................................................... 49

3.5. Summary of Glass Waste Form Dissolution Modeling .................................................. 49

4. MODELING AND SIMULATION OF CONTINUUM PROCESSES ............................... 53

4.1. Thermal-Hydrologic-Chemical Processes and Code Capabilities ................................ 53

4.1.1. Thermal Modeling ....................................................................................... 53

4.1.2. Hydrologic Modeling .................................................................................. 54

4.1.3. Multicomponent Multiphase Reactive-Transport Modeling ……………...... 55

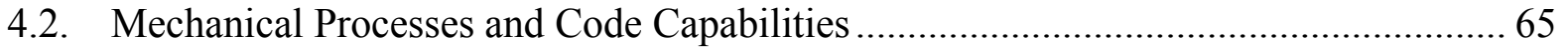

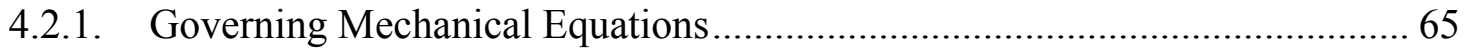

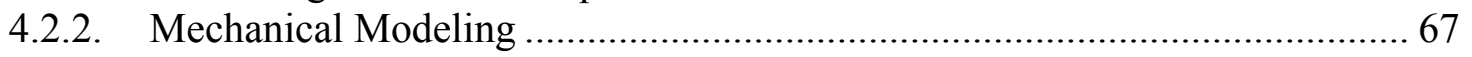

4.3. Preliminary Gap Analysis of THCM Code Capabilities................................................. 67 


\section{CONTENTS (cont.)}

5. FRAMEWORKS AND INFRASTRUCTURE.............................................................. 73

5.1. Enabling Infrastructure and Foundational Services ............................................. 73

5.1.1. Configuration Management ................................................................ 74

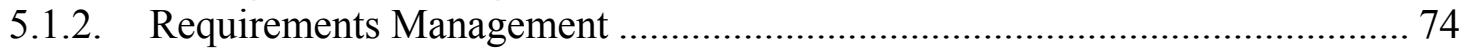

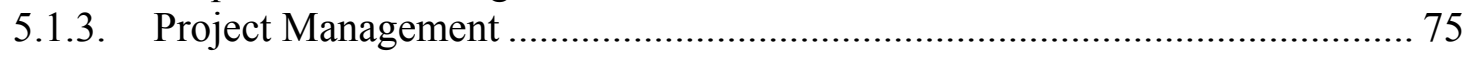

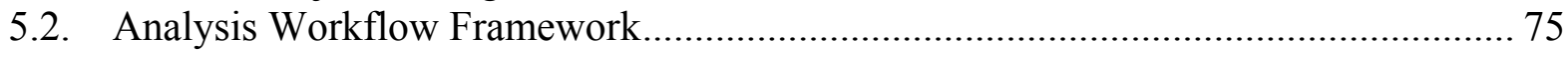

5.2.1. Plan for Gap Analysis, Acquisition, and Development ............................. 75

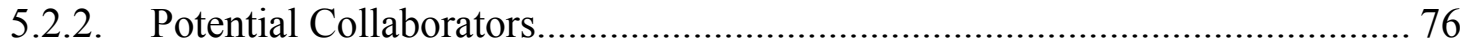

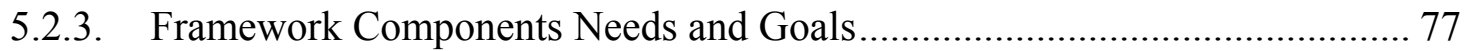

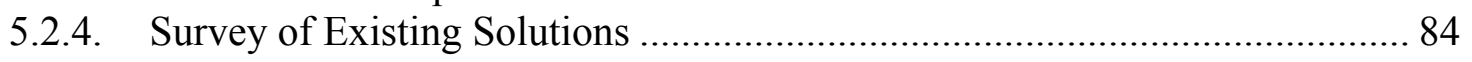

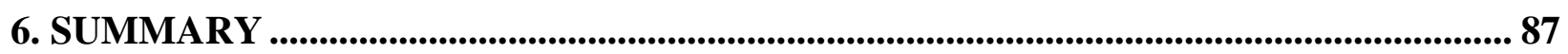

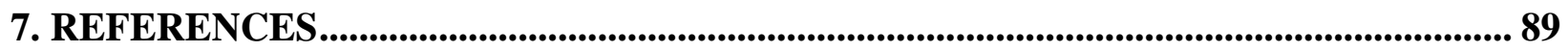

APPENDIX A: REACTIVE TRANSPORT CODES ................................................... 1 


\section{FIGURES}

Figure 2-1. Components of a generic disposal system. ................................................. 4

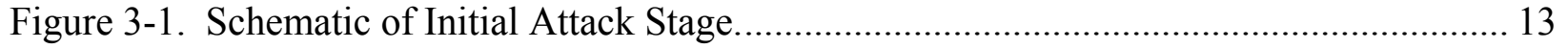

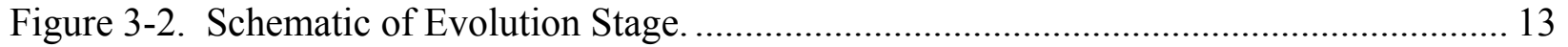

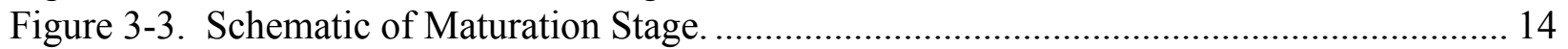

Figure 3-4. Time dependent alteration rate and extent for glass degradation.......................... 15

Figure 3-5. Schematic showing the compositional profiles through the layers on the glass

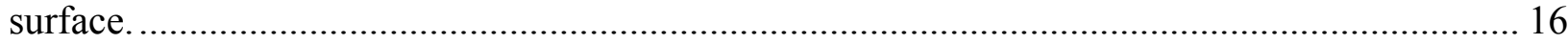

Figure 3-6. Schematic diagram of the processes occurring within the passivating reactive

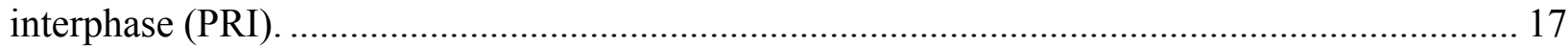

Figure 3-7. Time and length scales of geochemical modeling. ........................................... 20

Figure 3-8. Energy profile $(\mathrm{kJ} / \mathrm{mol})$ of the $\mathrm{Si}-\mathrm{O}-\mathrm{Si}$ hydrolysis reaction along the reaction coordinates for the protonated, neutral, and deprotonated species........................................ 28

Figure 3-9. Schematic of the Al- $\mathrm{O}_{\mathrm{br}}-\mathrm{Si}$ surface site in (a) protonated, (b) neutral, and

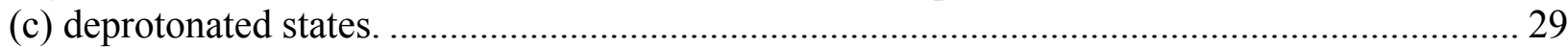

Figure 3-10. Flowchart for steps involved in the stepwise dissolution algorithm................... 35

Figure 3-11. Simplified model of a dissolving feldspar surface......................................... 39

Figure 5-1. Flow of modeling and simulation capabilities from Development........................ 73 


\section{TABLES}

Table 2-1. Groupings of Potential Waste Form Types ...................................................... 3

Table 2-2. Groupings of Potential Disposal Concepts and Geologic Settings ........................... 4

Table 5-1. Enabling Infrastructure - Tool Identification and Gap Analysis ............................. 74 


\section{ACRONYMS}

$\begin{array}{ll}\text { AFM } & \text { atomic force microscopy } \\ \text { ASC } & \text { Advanced Simulation and Computing } \\ \text { ASCEM } & \text { Advanced Simulation Capability for Environmental Management } \\ \text { CP-MAS } & \text { cross-polarization magic-angle spinning } \\ \text { CT } & \text { Capability Transfer } \\ \text { DOE } & \text { U.S. Department of Energy } \\ \text { EBS } & \text { Engineered Barrier System } \\ \text { ECT } & \text { Enabling Computational Technologies } \\ \text { FEP } & \text { feature, event, and process } \\ \text { FY } & \text { fiscal year } \\ \text { FMM } & \text { Fundamental Methods and Models } \\ \text { GTCC } & \text { greater than class C waste } \\ \text { HLW } & \text { high-level waste } \\ \text { HPC } & \text { high performance computing } \\ \text { HTGR } & \text { high-temperature gas-cooled reactor } \\ \text { IPSC } & \text { Integrated Safety and Performance Codes } \\ \text { LHS } & \text { Latin hypercube sampling } \\ \text { LTHLW } & \text { lower than high-level waste } \\ \text { MC } & \text { Monte Carlo } \\ \text { MD } & \text { molecular dynamics } \\ \text { MDCF } & \text { multi-mechanism deformation coupled fracture } \\ \text { MO-TST } & \text { molecular orbital-transition state theory } \\ \text { MPP } & \text { massively parallel processing } \\ \text { NE } & \text { Nuclear Energy } \\ \text { NEAMS } & \text { Nuclear Energy Advanced Modeling and Simulation } \\ \text { NMR } & \text { nuclear magnetic resonance } \\ \text { ODE } & \text { ordinary differential equation } \\ \text { PA } & \text { performance assessment } \\ \text { PDE } & \text { partial differential equation } \\ \text { PRI } & \text { passivating reactive interphase } \\ \text { RI } & \text { reactive interface } \\ \text { SEM } & \text { scanning electron microscopy } \\ \text { SIA } & \text { Sequential iterative approach } \\ \text { SIMS } & \text { secondary ion mass spectrometry } \\ \text { SNIA } & \text { Sequential non-iterative approach } \\ \text { SNL } & \text { Sandia National Laboratories } \\ \text { THC } & \text { thermal-hydrologic-chemical } \\ \text { THCM } & \text { thermal-hydrologic-chemical-mechanical } \\ \text { THCMBR } & \text { thermal-hydrologic-chemical-mechanical-biological-radiological } \\ \text { TST } & \text { transition state theory } \\ \text { UFD } & \text { Used Fuel Disposition } \\ \text { UNF } & \text { used nuclear fuel } \\ \text { UQ } & \text { uncertainty quantification } \\ & \\ & \\ \text { HE } & \end{array}$




\section{ACRONYMS (cont.)}

V\&V verification and validation

VSI vertical scanning interferometry

VU

Verification and Validation and Uncertainty Quantification

WIPP Waste Isolation Pilot Plant

XPS

YMP

X-ray photoelectron spectroscopy

Yucca Mountain Project 


\section{INTRODUCTION}

The U.S. Department of Energy (DOE) Office of Nuclear Energy (NE) Advanced Modeling and Simulation Campaign co-ordinates the development of Integrated Performance and Safety Codes (IPSCs) in four technical areas: Fuels; Reactors; Safeguards and Separations; and Waste. Within the DOE-NE Advanced Modeling and Simulation (NEAMS) Campaign, these four IPSCs are supported by four cross-cutting elements: Fundamental Methods and Models (FMM), Verification and Validation and Uncertainty Quantification (VU), Enabling Computational Technologies (ECT), and Capability Transfer (CT). The goal of the NEAMS Waste IPSC is to develop an integrated suite of computational modeling and simulation capabilities to quantitatively assess the long-term performance of waste forms in the engineered and geologic environments of a radioactive waste storage or disposal system. The Waste IPSC will provide this simulation capability (1) for a range of disposal concepts, waste form types, engineered repository designs, and geologic settings, (2) for a range of time scales and distances, (3) with appropriate consideration of the inherent uncertainties, and (4) in accordance with robust verification, validation, and software quality requirements.

In fiscal year 2010 (FY10), activities within the Waste IPSC included: (1) specification of a challenge problem and associated milestones to demonstrate proof of concept; (2) development of a verification and validation (V\&V) plan; (3) initial development and integration of thermalhydrologic-chemical-mechanical (THCM) code capabilities; and (4) initial development and integration of frameworks and enabling tools/infrastructure. These activities build upon the Waste IPSC system design specifications outlined in SNL (2009). The challenge problem specifications are documented in Freeze et al. (2010) and the V\&V plan is documented in Edwards et al. (2010). This report describes the progress in FY10 in the other two activities, the development and integration of (a) THCM code capabilities, and (b) frameworks and enabling tools and infrastructure.

The current Waste IPSC strategy is to acquire and integrate the necessary Waste IPSC capabilities wherever feasible, and develop only those capabilities that cannot be acquired or suitably integrated, verified, or validated. The development of the Waste IPSC will be an iterative process over the multi-year duration of the project. Further development and integration of code capabilities, frameworks, and tools will be documented in future reports.

\subsection{Waste IPSC Overview}

The overarching goal of the Waste IPSC is to develop an integrated suite of modeling and simulation capabilities to quantitatively assess the long-term performance of waste forms in the engineered and geologic environments of a radioactive waste storage or disposal system (SNL 2009, Section 1). This requires the simulation of the coupled thermal-hydrologic-chemicalmechanical-biological-radiological (THCMBR) processes that govern radionuclide (or other hazardous constituent) movement from the waste forms through the engineered components and the geosphere for a range of alternative disposal system designs (e.g., disposal concept, waste emplacement geometry, waste form type, engineered component designs, geologic setting) and conditions (e.g., saturated vs. unsaturated flow, boiling vs. non-boiling temperature, reducing vs. 
oxidizing chemistry). Accurate simulation of a disposal system requires modeling the coupled THCMBR processes over a broad range of time scales (nanoseconds to millions of years) and distances (angstroms to kilometers). The broad range of time scales and distances further requires the application of uncertainty quantification (UQ) techniques to the models and their inputs.

To achieve these goals, the Waste IPSC will incorporate three levels of model fidelity: constitutive relationships derived from mechanistic sub-continuum processes; high-fidelity continuum models; and moderate-fidelity performance assessment (PA) continuum models. The integration of modeling and simulation capabilities at these three levels of fidelity will derive from a combination of existing code acquisition and new code development. These multifidelity modeling and simulation capabilities must be supported by efficient frameworks and enabling tools/infrastructure, also derived from a combination of existing and new codes. Waste IPSC technical requirements are described in Freeze et al. (2010, Section 2), use cases are identified in SNL (2009, Section 5) and Freeze et al (2010, Section 3), and computational requirements are outlined in Edwards et al. (2010, Sections 3 through 6).

The remainder of this report describes the current (FY10) status of the development and integration of (1) THCM ${ }^{1}$ code capabilities and (2) frameworks codes and enabling tools and infrastructure. The development and integration activities to date have primarily focused on gap analyses to identify whether or not capabilities of existing codes meet the technical and computational requirements and to identify where new code development may be necessary. Section 2 summarizes the scope of the Waste IPSC to provide the technical requirements for the code identification and gap analysis. Section 3 describes the progress in characterizing subcontinuum processes. Section 4 describes the progress in identifying applicable codes, and associated gaps, for the high-fidelity-scale and PA-scale continuum models. Section 5 summarizes the Waste IPSC computational requirements describes the progress in identifying applicable codes, and associated gaps, for the frameworks and infrastructure. Section 6 summarizes the overall Waste IPSC progress to date.

The Waste IPSC gap analysis is a continuing process and the analysis documented in this report serves only as a starting point for a full analysis planned to be conducted in future years. As a result, the list of codes examined in this report is by no means exhaustive.

\footnotetext{
${ }^{1}$ The final Waste IPSC will include consideration of THCMBR capabilities and couplings. However, as is discussed in Section 2, only THCM capabilities and couplings were considered in FY10.
} 


\section{WASTE IPSC TECHNICAL SCOPE}

As described in Section 1.1, the Waste IPSC will provide the capabilities to quantitatively assess the long-term performance of a radioactive waste storage or disposal system, including both the engineered and geologic environments, based on the coupled THCMBR processes that govern radionuclide (or other hazardous constituent) movement through the entire disposal system. The Waste IPSC will enable simulation of disposal system performance at three different model fidelities (sub-continuum scale, high-fidelity continuum scale, and PA continuum scale) for a range of candidate waste forms, disposal concepts and designs, engineered and geologic environments, and associated conditions over a broad range of time and length scales. The multi-fidelity development of the Waste IPSC will incorporate capabilities for characterizing material properties (e.g., chemical reactions and chemical kinetics) at the sub-continuum scale, upscaling into constitutive equations at the continuum scale, developing high-fidelity models of coupled THCMBR processes (e.g., reactive transport), and abstracting the coupled processes into computationally efficient PA models for quantitative assessment of disposal system performance.

In collaboration with the DOE-NE Used Fuel Disposition (UFD) Campaign, a set of 6 potential waste form type groupings (Table 2-1) and 8 potential disposal concept/geologic setting groupings (Table 2-2) were identified to define the expected range (based on current knowledge) of disposal system concepts, designs, settings and conditions (Freeze et al. 2010, Section 2.1; Freeze et al. 2010b, Section 2.1).

Table 2-1. Groupings of Potential Waste Form Types

\begin{tabular}{|c|l|l|}
\hline Group Number & Waste Form Type & Description \\
\hline 1 & Used Nuclear Fuel (UNF) & e.g., Commercial, DOE-Owned, HTGR \\
\hline 2 & $\begin{array}{l}\text { High-Level Waste (HLW) } \\
\text { Glass }\end{array}$ & $\begin{array}{l}\text { Current (e.g., borosilicate) and future (e.g., no } \\
\text { minor actinides) }\end{array}$ \\
\hline 3 & $\begin{array}{l}\text { High-Level Waste (HLW) } \\
\text { Glass Ceramic / Ceramic }\end{array}$ & $\begin{array}{l}\text { Current (glass bonded sodalite) and future } \\
\text { (e.g., from electrochemical processing) }\end{array}$ \\
\hline 4 & $\begin{array}{l}\text { High-Level Waste (HLW) } \\
\text { Metal Alloy }\end{array}$ & $\begin{array}{l}\text { From electrochemical or aqueous } \\
\text { reprocessing, cermets }\end{array}$ \\
\hline 5 & $\begin{array}{l}\text { Lower Than HLW (LTHLW) } \\
\text { Class A, B, and C, and GTCC }\end{array}$ \\
\hline 6 & Other & $\begin{array}{l}\text { Molten salt, electro-chemical refining waste, } \\
\text { etc. }\end{array}$ \\
\hline
\end{tabular}

Note: HTGR = High-temperature gas-cooled reactor; GTCC = Greater than Class C. 
Table 2-2. Groupings of Potential Disposal Concepts and Geologic Settings

\begin{tabular}{|c|l|l|}
\hline Group Number & Disposal Concept / Geologic Setting & Description \\
\hline 1 & Surface Storage & $\begin{array}{l}\text { Long-term interim storage at reactors or at } \\
\text { centralized sites }\end{array}$ \\
\hline 2 & Shallow Disposal & $\begin{array}{l}\text { e.g., near-surface disposal, LTHLW sites } \\
\text { (Depths <= 100 m) }\end{array}$ \\
\hline 3 & $\begin{array}{l}\text { Mined Geologic Disposal } \\
\text { (Hard Rock, Unsaturated) }\end{array}$ & $\begin{array}{l}\text { Granite/crystalline or tuff } \\
\text { (Depths }>100 \mathrm{~m} \text { ) }\end{array}$ \\
\hline 4 & $\begin{array}{l}\text { Mined Geologic Disposal } \\
\text { (Hard Rock, Saturated) }\end{array}$ & $\begin{array}{l}\text { Granite/crystalline or tuff } \\
\text { (Depths }>100 \mathrm{~m} \text { ) }\end{array}$ \\
\hline 5 & $\begin{array}{l}\text { Mined Geologic Disposal } \\
\text { (Clay/Shale, Saturated) }\end{array}$ & $\begin{array}{l}\text { Clay/shale } \\
\text { (Depths }>100 \mathrm{~m} \text { ) }\end{array}$ \\
\hline 6 & $\begin{array}{l}\text { Mined Geologic Disposal } \\
\text { (Salt, Saturated) }\end{array}$ & $\begin{array}{l}\text { Bedded or domal salt } \\
\text { (Depths }>100 \mathrm{~m})\end{array}$ \\
\hline 7 & Deep Borehole Disposal & $\begin{array}{l}\text { Granite/crystalline } \\
\text { (Depths } 1000 \text { m) }\end{array}$ \\
\hline 8 & Other & Sub-seabed, carbonate formations, etc. \\
\hline
\end{tabular}

The groupings in Table 2-1 and Table 2-2 are described in more detail in Freeze et al. (2010b, Sections 2.1.1 and 2.1.2). These groupings result in 35 combinations (ignoring the placeholder "Other" groups) of waste form types and disposal concepts/geologic settings that broadly define the range of potential alternative disposal system designs that might need to be evaluated using the Waste IPSC. Within any single alternative disposal system design there may be important sub-designs (e.g., waste emplacement geometry, thermal loading, engineered component (waste form, waste package, backfill, etc.) design and materials) and/or conditions (e.g., saturated vs. unsaturated flow, boiling vs. non-boiling temperature, reducing vs. oxidizing chemistry) that may further delineate the range of technical capabilities required of the Waste IPSC.

Figure 2-1 shows a conceptualization of a generic disposal system that includes components, domains, and phenomena common to most of the 35 disposal system alternatives and that therefore need to be encompassed by the Waste IPSC modeling and simulation capabilities (Freeze et al. 2010, Section 2.1.1).

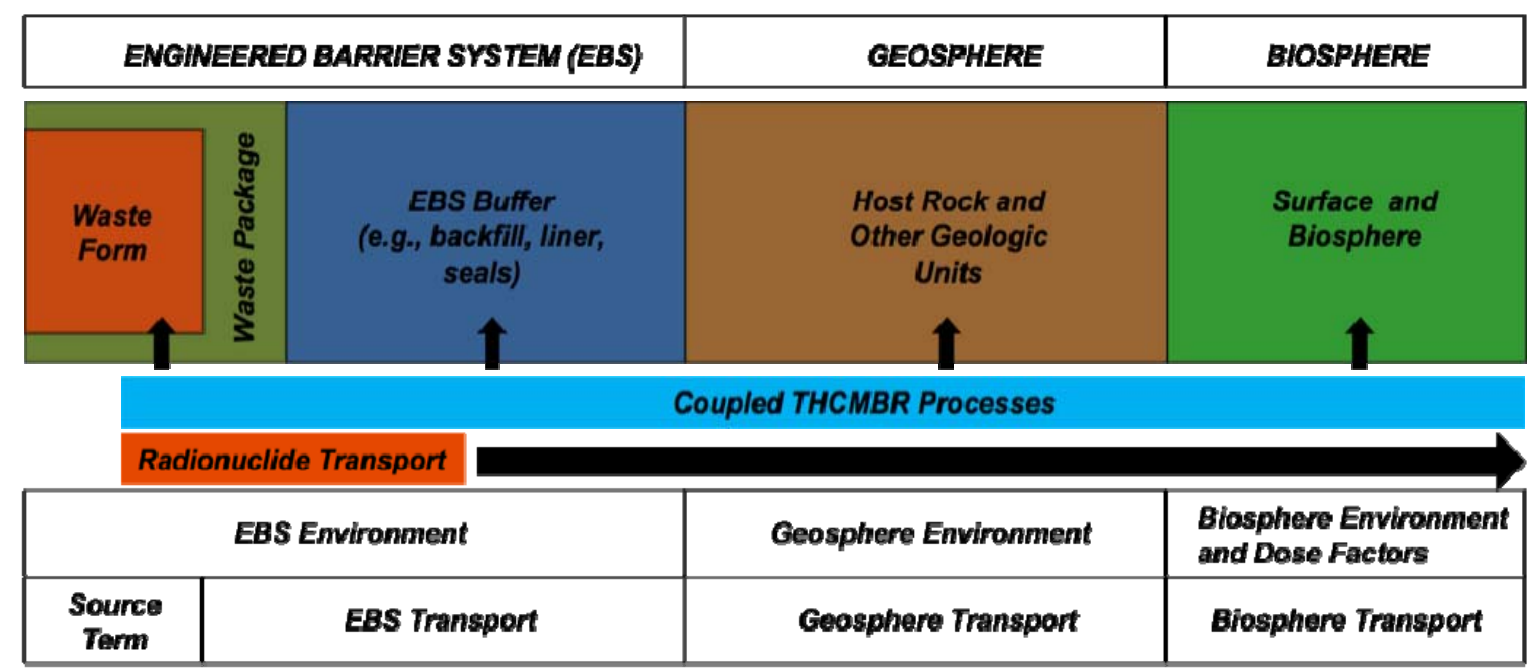

Figure 2-1. Components of a generic disposal system. 


\section{The top half of}

Figure 2-1 shows the physical domains of the generic disposal system: engineered barrier system (EBS), geosphere, and biosphere. The terms near field (or near-field environment) and far field (or far-field environment) are also commonly used to describe the physical domains of a disposal system. The near field encompasses the EBS and well as the interface with, and adjacent portion of, the host rock that experiences durable (but not necessarily permanent) changes due to the presence of the repository (e.g., hydro-mechanical alteration due to tunnel excavation, thermalchemical alteration due to waste emplacement). The far field encompasses the remainder of the geosphere and the biosphere.

\section{The bottom half of}

Figure 2-1 shows the phenomena that can affect each of these domains. These phenomena include, at a high level, the coupled THCMBR processes that control:

1) Radionuclide Source Term - which includes degradation of the waste form, degradation of the waste package, interaction with the EBS environment, and radionuclide solubility,

2) Radionuclide Transport through the EBS - which includes advection, diffusion, and sorption of dissolved and colloidal radionuclides and interaction with the EBS environment,

3) EBS Environment - which includes temperature, fluid movement, fluid chemistry, biology, and mechanical degradation of EBS components (e.g., waste form, waste package, buffer materials, backfill, liner, seals),

4) Radionuclide Transport through the Geosphere (the host rock and surrounding geologic units) - which includes advection, diffusion, and sorption of dissolved and colloidal radionuclides and interaction with the geosphere environment,

5) Geosphere Environment - which includes temperature, fluid movement, fluid chemistry, biology, and mechanical alteration of the host rock and surrounding geologic units,

6) Radionuclide Transport in the Biosphere - which includes radionuclide movement, uptake, and human health effects, and,

7) Biosphere Environment and Dose Factors - which includes THCMBR processes that affect radionuclide movement and uptake and the characteristics of receptor.

These high-level phenomena are represented and described in additional detail by a set of potentially relevant features, events, and processes (FEPs). The identification of a preliminary set of 208 Waste IPSC FEPs broadly applicable across the range of 35 disposal alternatives is described in Freeze et al. (2010b, Section 2.3).

The technical scope of the Waste IPSC must be broad enough to represent the range of potentially relevant THCMBR processes (and associated time- and length-scales) captured by 
these 7 high-level phenomena for a range of disposal system alternatives encompassed by the 35 combinations of concepts/settings and waste form types.

Because FY10 was the first year of Waste IPSC development and integration, a necessary initial step was the identification of existing code capabilities, and gaps in existing capabilities, relevant to the modeling and simulation of the Waste IPSC phenomena and disposal alternatives. The initial code identification and gap analysis, performed in FY10, focused on a subset of the 7 high-level phenomena and 35 disposal alternatives. In future years the full set of phenomena and disposal alternatives will be considered.

The subset of high-level phenomena and disposal alternatives considered in FY10 are described below:

- The FY10 gap analysis focused on THCM processes and couplings. While, the final scope of the Waste IPSC will include coupled THCMBR processes, the gap analysis for biological and radiological code capabilities (the BR component of THCMBR) was deferred to future years. The effects of some biological and radiological processes are indirectly captured within the THCM processes.

- The FY10 gap analysis focused on code capabilities to model the 3 high-level phenomena relevant to the EBS (Source Term, EBS Environment, and EBS Transport). The only EBS component considered for degradation was the waste form. Geosphere phenomena (Geosphere Environment and Geosphere Transport) were considered where code capabilities for the EBS phenomena were also applicable to the geosphere. In general, THCM processes and couplings are most complex in the EBS and decrease in complexity with distance from the waste. Gap analysis of code capabilities for other EBS degradation phenomena, other geosphere phenomena, and for biosphere phenomena was deferred to future years.

- The FY10 gap analysis focused on one waste form type: HLW Glass. Consideration of UNF, HLW Ceramic, HLW Metal, and LTHLW waste forms were deferred to future years.

- The FY10 gap analysis focused on four disposal concepts/geologic settings: Mined Geologic Disposal in saturated conditions for three geologic settings (hard rock, clay/shale, salt) and Deep Borehole Disposal. Consideration of Surface Storage, Shallow Disposal, and Mined Geologic Disposal in unsaturated conditions (hard rock) concepts were deferred to future years.

It was also necessary to ensure that the FY10 gap analysis considered code capabilities required for the Waste IPSC challenge problem and milestones (Freeze et al. 2010, Section 3) specifically a HLW borosilicate glass waste form in a mined geologic disposal system in salt.

In summary, the FY10 gap analysis considered code capabilities to model 5 high-level THCM phenomena for 4 disposal alternatives ( 1 waste form times 4 concepts/settings). These code capabilities were considered at both the sub-continuum scale and the continuum scale (for both high-fidelity and PA applications). 
Section 3 describes the identification and gap analysis of constitutive relationships derived from mechanistic sub-continuum processes potentially relevant to one of the high-level THCM phenomena: Radionuclide Source Term. The specific focus for FY10 was on HLW glass waste form degradation, with only minor consideration of EBS Environment. For FY10, this subcontinuum gap analysis was independent of disposal concept/geologic setting.

Section 4 describes the identification and gap analysis of continuum code capabilities to model four high-level THCM phenomena: EBS Environment, EBS Transport, Geosphere Environment, and Geosphere Transport. These code capabilities were considered for applicability to the 4 disposal concepts/geologic settings noted above: Mined Geologic Disposal in saturated hard rock, clay/shale, and salt, and Deep Borehole Disposal. Considerations for mined disposal in hard rock and deep borehole disposal are similar. For FY10, this continuum code gap analysis was independent of waste form type.

As technologies and socio-political drivers evolve, the relevant waste form groupings, concept/setting groupings, disposal system domains, and phenomena may all evolve, which may in turn lead to evolving code requirements. 


\section{CHARACTERIZATION OF SUB-CONTINUUM PROCESSES}

As described in Section 2, the consideration of sub-continuum processes for the Waste IPSC in FY10 was limited to one of the high-level THCM phenomena: Radionuclide Source Term. The specific source term focus was on HLW glass waste form degradation.

The rate at which the waste form degrades has a potential to improve estimated performance of repositories if the resulting waste-form lifetime is sufficiently long relative to the period of performance (e.g., on the order of hundreds of thousands of years), and if the waste form effectively immobilizes those radionuclides that will be most mobile in the proposed geologic setting (Swift et al. 2010). Therefore, the capability to simulate waste form degradation and its effect on the time-dependent source term is an important component of any disposal system assessment.

Characterizing the chemical and structural processes that lead to waste form degradation, radionuclide release, and reactive transport involves modeling phenomena at the sub-continuum scale. Abstracting, "upscaling", these mechanistic, atomistic processes into effective collective phenomena formulated into constitutive equations with validated parameterizations is required to inform coupled THCM continuum simulations of the long-range and long-time performance of waste disposal systems. The goal of the sub-continuum and upscaling area is to establish and demonstrate the capabilities necessary to model each link in the chain of sub-continuum phenomena, and establish the linkages between hierarchies within the sub-continuum domain that allow for a quantitative propagation of the atomistic phenomena into constitutive models, with appropriate evaluations of the associated uncertainties. These phenomena will need to account for different waste form types, temperature and chemical conditions. The achievement of a quantitatively connected hierarchical chain of models entails overcoming significant scientific and modeling challenges. This section describes the nature of those challenges and gaps for the evaluation of source terms for degrading waste forms at the sub-continuum-tocontinuum scale.

The Waste IPSC will ultimately enable capabilities encompassing the various waste form types listed in Table 2-1. For this report, describing FY10 progress, only HLW glass has been considered. As discussed below, the approach used here for HLW glass will be the template for addressing the other waste form types similarly in the future.

Borosilicate glass is the specific target of the Waste IPSC challenge milestone 2 (Freeze et al. 2010, Section 3.2). Establishing the end-to-end hierarchical chain of atoms-to-continuum modeling of glass dissolution is a major goal of FY11 activities at the sub-continuum scale. A detailed description of the gaps is timely; it is necessary for executing immediate activities within the Waste IPSC and to provide early guidance to the FMM, VU, and ECT supporting program elements as to the needs of the Waste IPSC.

Specific gap assessments for other waste form types, e.g., ceramics, metal alloys, or UNF, will be prepared as those efforts are developed and will be documented in future reports. At the subcontinuum scale, many phenomena do not distinguish between waste form types, e.g., activation barriers for simple atomic chemical processes in different materials are evaluated with similar 
methods. Many of the processes described for glass corrosion apply to ceramics, and also to metal alloy waste forms, and the same capability gaps are common to all. Characterization of sub-continuum phenomena for UNF will likely leverage ongoing activities in the NEAMS Fuels IPSC and FMM program element because the distinguishing needs for the Waste IPSC, the chemical interactions of UNF with the EBS environment, are fundamentally similar to the same processes for the other candidate waste forms.

The gap analysis at the sub-continuum scale will focus on scientific capabilities rather than code deficiencies. There is an abundance of modeling codes applicable to the sub-continuum scale. The gaps in enabling capabilities for the Waste IPSC lie in designing meaningful simulations for complex processes and quantitatively propagating results of simulations through a hierarchy of phenomena into constitutive relations that can be used in continuum-scale simulations.

\subsection{Overview of Glass Waste Form Dissolution}

\subsubsection{Repository Settings - Why and When Glass Waste Form Dissolution is Important}

Understanding the detailed mechanisms that lead to the disparity in rates between far-from equilibrium dissolution of glass measured in the laboratory and the fully-coupled degradation of glass observed in natural systems can provide insight into estimates of lifetimes for glass radioactive waste forms. The rates of glass degradation observed from ancient glass artifacts are much slower than those observed in laboratory tests (Verney-Carron et al. 2010), similar to the slower degradation rates observed in nature for mineral degradation (White and Brantley 2003; Ganor et al. 2005; Hellmann and Tisserand 2006; Bryan et al. 2009). Studies of natural and synthetic glass dissolution rates show far-from equilibrium behavior can be compositionally dependent on Si content (Wolf-Boenisch et al. 2004) and Al content (Hamilton et al. 2001) of the glass. The slower degradation glass rates observed in the natural system result from a number of differences between those systems and glass studies in the laboratory including (a) dissolution mechanisms (Hamilton et al. 2001) and (b) glass reactive surface area (Wolf-Boenisch et al. 2004). Each of these aspects of the degradation are affected by coupled feedback processes resulting during glass degradation due to formation of diffusion pathways through intermediate phases (e.g., gel layer), which may also develop to isolate fresh glass surfaces from contact with the bulk fluid (Cailleteau et al. 2008; Verney-Carron 2010).

Glass waste forms have been evaluated for permanent disposal in radioactive waste disposal facilities (i.e., geologic repositories) in a number of countries. For example, the DOE assessed a geologic repository at the Yucca Mountain site (DOE 2009) that included disposal of both spent nuclear fuel and high-level radioactive waste in an unsaturated fractured volcanic tuff characterized by oxidizing conditions. In addition, both the French (ANDRA 2005) and Swiss (NAGRA 2002) national programs have evaluated disposal of spent nuclear fuel and high-level radioactive waste in a clay repository where conditions are anticipated to be water-saturated and chemically reducing, similar to approaches also being evaluated in Belgium and other countries.

The relatively long duration of glass waste forms (a few hundred thousand years) in the performance analysis of the French clay site (ANDRA 2005) provides substantial delay to peak radionuclide releases to the biosphere. This is demonstrated through sensitivity analyses using 
constant glass degradation rates through time, rather than decreasing degradation rates resulting from increasing silica saturation in solution (Swift et al. 2010). Rates of glass waste form degradation used in the DOE analyses of the unsaturated Yucca Mountain site reflect the farfrom equilibrium glass dissolution rates that conservatively do not include any of the coupled chemical processes that can decrease those rates (DOE 2009).

Further understanding of the detailed mechanisms of glass degradation will facilitate incorporation of more comprehensive and realistic representations of glass waste form behavior and provide further confidence in safety assessments of radioactive waste disposal. This report summarizes existing approaches and identifies a number of areas for further work focusing on topics that should advance the ability to upscale from molecular-scale approaches of glass behavior to models of glass degradation at the continuum-scale.

\subsubsection{Context of Glass Degradation}

This section provides a multiscale conceptual model as a framework for modeling glass waste forms. This framework is used to describe both the physical and chemical processes and the spatial and temporal scales, primarily related to the continuum model scale, and is used as a means to delineate the spatial and temporal relevancy of specific chemical processes to atomistic scale modeling approaches.

The progression of glass degradation in contact with an aqueous solution is a complex evolving set of reactions and transport mechanisms that occur at various and variable rates depending on the stage of the glass degradation. The primary controls on the overall rate change throughout this progression depend on both the physical and chemical evolution of the system at the molecular and continuum scales. Within the degrading system, a series of coupled chemical processes proceeds to change the initial simplistic glass-solution reaction into a multi-layer, multiphase reactive transport regime that produces a range of effects including diffusion-rate controls, altered water composition in contact with the reactive interface of the glass, and physical modification of the surface area of the reactive interface. Understanding these processes in a coherent manner facilitates the consistent extraction of glass reaction rate information from experimental work, provides the context for constraining the long-term evolution of glass waste forms in a repository environment, and delineates a framework for models of glass degradation to be consistently developed and upscaled from the atomistic/molecular level to high-fidelity continuum scale, and from there to the PA scale.

For the purposes of the discussion in this report, the following definitions are given here to ensure clarity.

Glass Dissolution - the direct reaction of an aqueous solution with fresh glass to dissolve the solid material.

Reactive Interface - the contact between the pristine glass and the aqueous solution with which it is reacting. It is the surface area of the reactive interface that should apply to the overall glass dissolution rate. 
Glass Degradation - the entire set of processes leading to alteration of glass to other phases (for glass waste this includes concurrent release of radioelements intended to be isolated in a geologic repository). Note that glass degradation includes glass dissolution as a sub process.

The process of glass degradation takes place in a series of continuous stages with the following primary distinctions made within this report:

Initial attack - the stage when the fresh glass surface is reacting with the solution and the glass dissolution process is dominant within the series of glass degradation processes.

Evolution - the stage when alteration layers (e.g., diffusion layer, gel layer, secondary phase layer) are forming on glass surfaces and growing at rates relative to one another. This is the period when the glass degradation rate slows from its initial rate.

Maturation - the stage when alteration layers are all formed and growing only at steady state relative to one another. This is the period when the degradation rate could approach a constant, long-term value.

The Initial Attack stage is delineated as the high rate, far from equilibrium portion of glass degradation and is dominated by the sub-process of glass dissolution (Figure 3-1). This is the period in the experimental studies prior to any significant effects of coupled processes on the rate of reaction. Temporally, this is the scale at which connections should be approached between atomic scale and continuum scale models of the rate of glass dissolution. The Evolution stage (Figure 3-2) has the most complex coupling of processes because the transport field is developing in a non-steady state manner such that the process-level rate limitations on the overall degradation rates are continuously changing their relative contributions. Once the Maturation stage is reached (Figure 3-3), steady state has been achieved within the formation of the gel and secondary phase layers so that the overall degradation rate is now dominated by the coupled processes that occur within this multiphase system. This latter stage would be more ideal for constraining the evaluation of the transport limitations on the system, but it is important to consider the variations in water composition throughout the system when performing an upscaling comparison from the atomistic level to the continuum level. 
$\mathrm{T}=\mathrm{T}_{0}$ to $\mathrm{T}_{1}$

Initial glass-solution contact and reaction

Dissolution rate (= degradation rate) is high

No intermediate phases developed

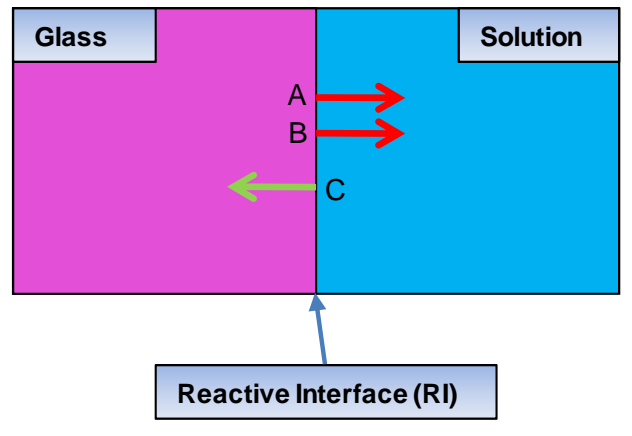

A - rate of alkali surface release

$\mathrm{B}$ - rate of $\mathrm{Si}$, Al surface detachment (tends to be limiting)

$\mathrm{C}$ - rate of $\mathrm{H}_{2} \mathrm{O}$ diffusion into glass

During the initial attack stage, the fresh glass surface is reacting with the solution and glass dissolution processes dominate.

\section{Figure 3-1. Schematic of Initial Attack Stage.}

$T=T_{1}$ to $T_{2}$

Active (non-steady state) growth of gel ( \pm secondary phases) layer(s)

Degradation rate is slowing

Transport through gel (etc.) layer affects rate

Aqueous composition at RI changing

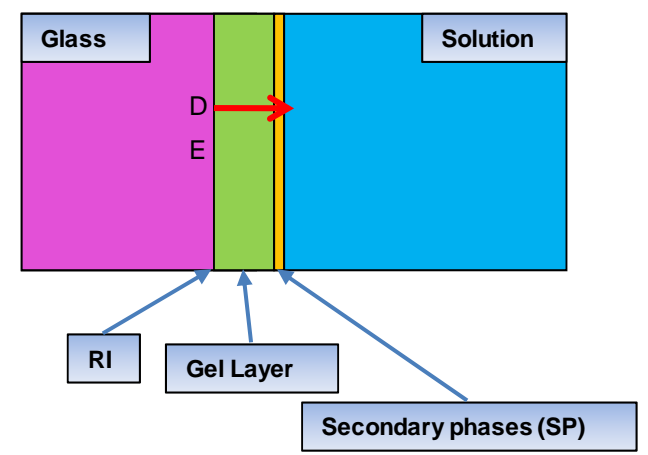

$D$ - rate of silica diffusion through the gel $( \pm$ SP) layer (at least partially limiting). Other constituents mass transport rates may also be important. $\mathrm{E}$ - the aqueous composition at the RI is not the same as the Solution (aqueous silica concentration especially) and the surface area of the RI may be reduced by glass-gel contact area.

During the evolution stage, alteration layers (e.g., gel layer, secondary phase layer) form on glass surfaces and grow at different rates relative to one another. This is the period when the glass degradation rate slows from its initial rate. This decrease in rate maybe due to diffusion through the layers forming on the glass surface, changes in aqueous composition at the reactive interface $(\mathrm{RI})$ of the glass, and changes in reactive surface area.

Figure 3-2. Schematic of Evolution Stage. 
$\mathrm{T}=\mathrm{T}_{2}$ to $\mathrm{T}_{3}$

Steady state growth of gel and secondary phases layers provide mantle on

glass

Degradation rate is very slow and steady (reaching plateau in this stage)

Transport through gel layer affects rate

Aqueous composition at RI is consistent but different than Solution

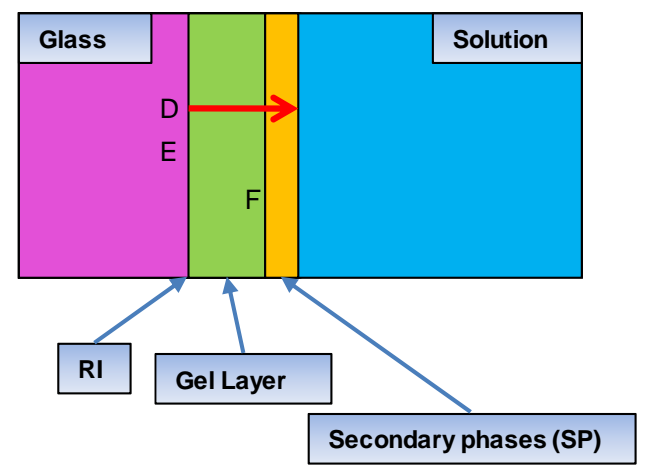

$D$ - rate of silica diffusion through the gel $( \pm$ SP) layer (at least partially limiting). Other constituents mass transport rates may also be important. $\mathrm{E}$ - the aqueous composition at the RI is not the same as the Solution (aqueous silica concentration especially) and the surface area of the RI may be reduced by glass-gel contact area.

$\mathrm{F}$ - the gel and secondary phase layers may be acting as a mantle, in part isolating the fresh glass from the Solution.

During the maturation stage, alteration layers are all formed and growing only at steady state relative to one another. This is the period when the degradation rate could approach a constant, long-term value. This slow long-term rate may be due to diffusion through the layers forming on the glass surface, changes in aqueous composition that exist at the reactive interface (RI) of the glass, and reduction in reactive surface area. In this case, the gel and secondary phase layers may act as mantling phases that potentially isolate the fresh glass from the solution.

\section{Figure 3-3. Schematic of Maturation Stage.}

In a recent evaluation of glass degradation studies (Van Iseghem et al. 2007) the time dependent alteration (i.e., degradation) rate and extent of glass degradation was summarized (Figure 3-4). Note that the Interdiffusion and Hydrolysis periods equate to the Initial Attack stage; the Diffusion and affinity period equates to the Evolution stage; and the Slow alteration period equates to the Maturation stage. This is based primarily on the release rate of $\mathrm{Si}$, which represents the structural glass network. For repository systems that are quiescent (i.e., little or no seismiscity with tightly packed materials), it may be possible for the glass waste form to remain within the Maturation stage for most of its lifetime. This would potentially extend the lifetime of the glass waste form to periods approaching the regulatory limits and could impact performance of those repository systems in a positive manner (Van Iseghem et al. 2006; Swift et al. 2010). 


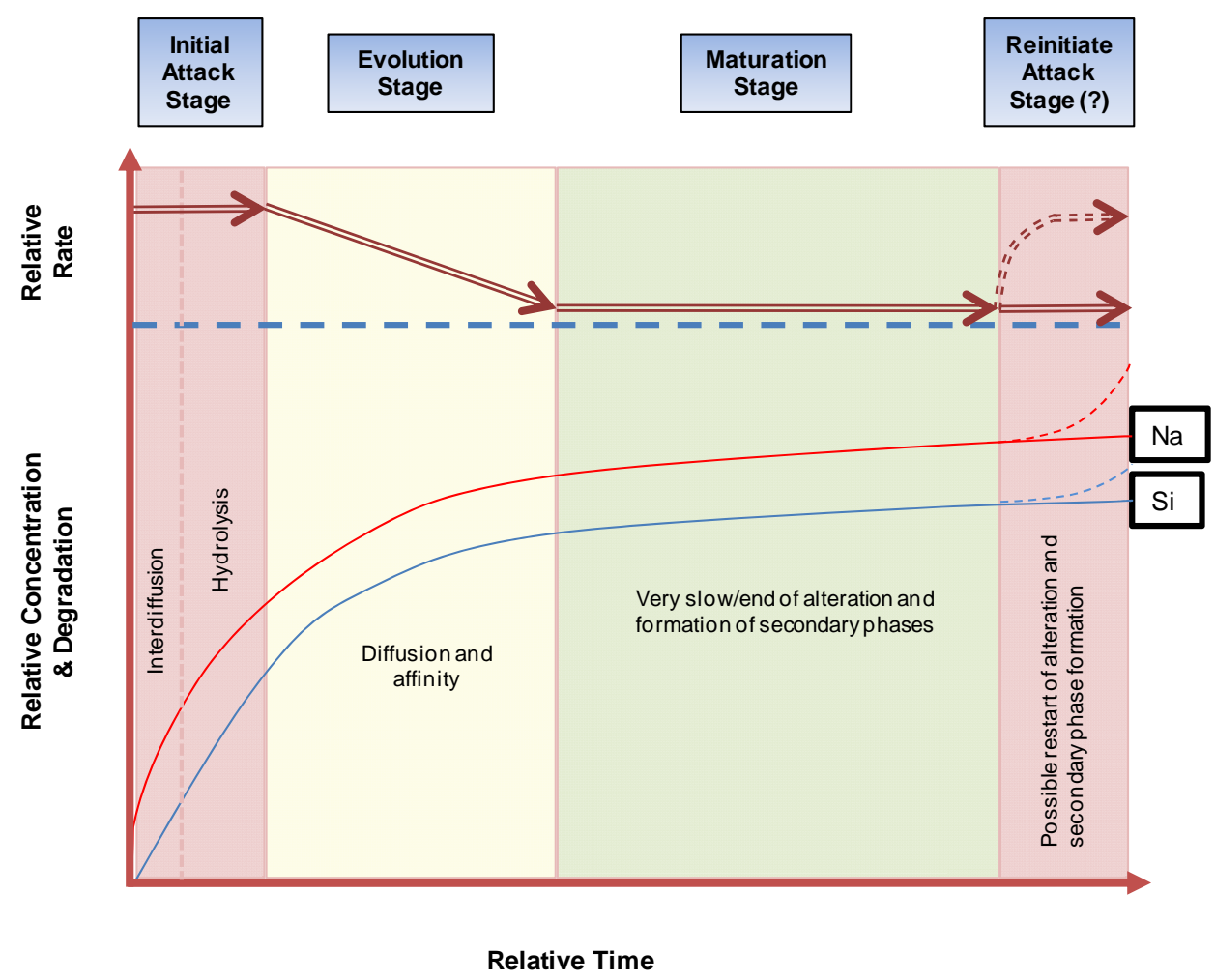

Note: Interdiffusion and Hydrolysis periods equate to Initial Attack stage; Diffusion and affinity period equates to Evolution stage; Slow alteration period equates to Maturation stage. (after Van Iseghem et al. 2007).

Figure 3-4. Time dependent alteration rate and extent for glass degradation.

\subsubsection{Current Understanding and Gap Identification}

A number of studies have summarized and/or evaluated the current information regarding glass degradation including experimental studies, field studies, and model development (Grambow 2006; Van Iseghem et al. 2006; Van Iseghem et al. 2007). The GLAMOR project (Van Iseghem et al. 2006; Van Iseghem et al. 2007) was a concerted effort to evaluate the primary evolving processes of glass degradation and to compare the capabilities of two specific models to represent those multiple processes using a selected set of experimental data from the literature. A number of more recent studies have extended glass degradation modeling from the end point of the GLAMOR project using the GRAAL model applied to SON68 nuclear glass (Frugier et al. 2008; Frugier et al. 2009) and explicitly evaluating coupled processes in the alteration of Roman glass (Verney-Carron et al. 2010). In these cases, the details of choosing parameter values for input to the model of these coupled processes emphasizes the facility of an integrated framework for evaluating experiments and analogs.

In the GRAAL model (Frugier et al. 2008; Frugier et al. 2009), the concept of the passivating reactive interphase (PRI) is developed (Figure 3-5); the PRI is essentially most of the gel layer and secondary phase layers that develop on the glass surface. The compositional profiles change rapidly just outside the surface of the glass (Figure 3-5) The PRI is primarily where the transport limitations occur. The PRI has depleted gel on one side and reacts directly with the solution on 
the other (Figure 3-6). Coupled diffusion and alteration processes in the PRI play a role in moderating the degradation rate of the glass (Frugier et al. 2009). At the field scale, understanding the feedback of the coupled processes that result in formation of the gel, and especially precipitation of secondary stable phases (e.g., clays) is central to elucidating the longtime slower rates observed for glass in nature (Verney-Carron et al. 2010) and will be central to application within PA models.

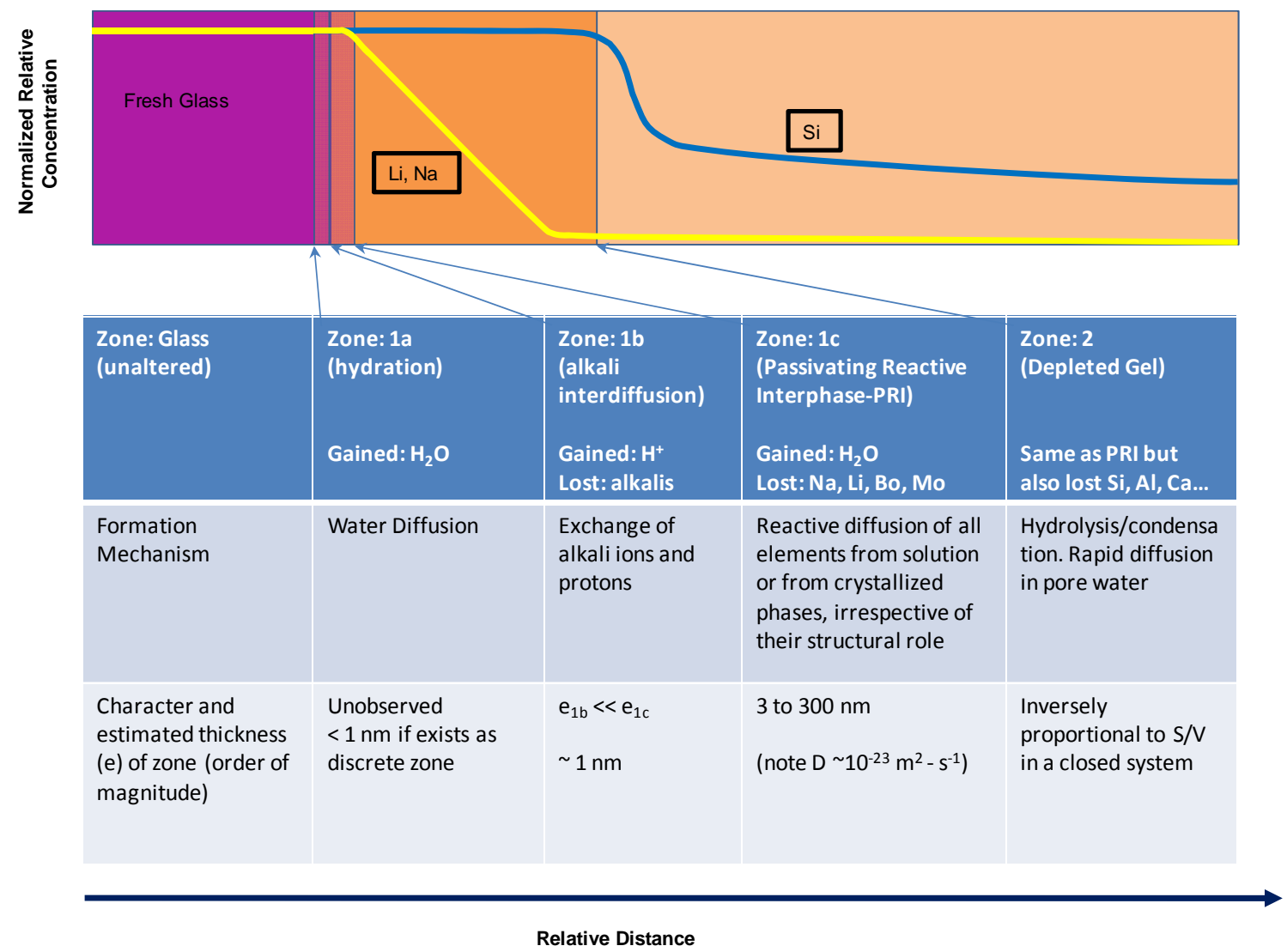

The layers shown include the passivating reactive interphase (PRI) and the depleted gel that contacts the solution. In the boxes below the schematic, the layers are listed with the primary formation mechanisms of these likely amorphous phases and the gross characteristics of each layer. (after Frugier et al. 2008).

Figure 3-5. Schematic showing the compositional profiles through the layers on the glass surface. 
Water diffusion through the PRI

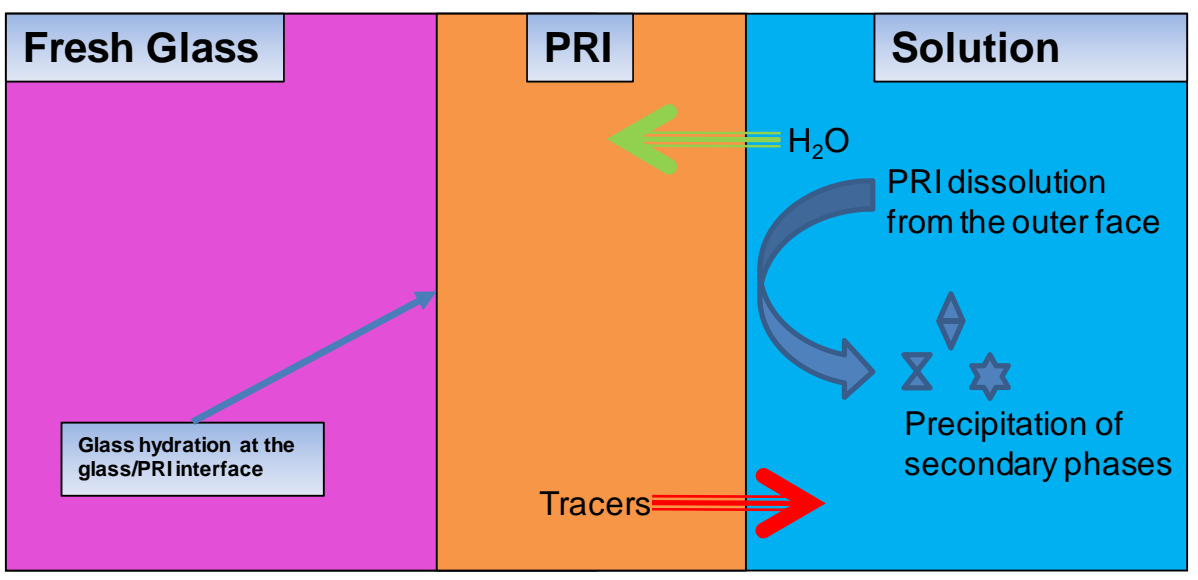

Shown above are the reactions of the PRI with the solution that are included in the GRAAL model of SON68 glass alteration (after Frugier et al. 2009).

Figure 3-6. Schematic diagram of the processes occurring within the passivating reactive interphase (PRI).

Experimental studies of mineral dissolution kinetics (e.g., Hamilton et al. 2001; Hellmann and Tisserand 2006; Yang and Steefel 2008; Pierce et al. 2010) and of the related process of glass degradation (e.g., Strachan and Croak 2000; Advocet et al. 2001; Wolf-Boenisch et al. 2004; Pierce et al. 2006; Cailleteau et al. 2008) have provided data to show that there is a complex coupled feedback process that changes glass degradation from simple far-from equilibrium dissolution kinetics at the start to slower degradation controlled in part by diffusion processes in gel and secondary phase layer(s) in which the reaction solution has a modified composition near the surface (i.e., the reactive interface) of fresh glass (see Grambow 2006; Van Iseghem et al. 2007). In these cases the compositional gradients of the solution need to be taken into account and evaluated on a smaller scale than the bulk solution ( $\mathrm{Li}$ et al. 2008) and the appropriate approach to developing models of this coupled reaction and transport system is in general a reactive transport methodology (e.g., Steefel et al. 2005). This allows capture of both far-from equilibrium dissolution processes of the initial attack stage for glass as well as the evolved system with secondary phases, transport process limitations, locally modified solution composition, and changes to reactive interface surface areas.

The areas below have been identified as primary gaps that have high potential for progress in order to (a) explicitly up-scale atomistic models to continuum models, (b) develop more precise models of the major processes at the continuum level, and/or (c) include more realistic long-term glass behavior in PA models. These gaps are not specific to just glass nuclear waste forms, but are gaps in synthesis/understanding of crystalline and amorphous material dissolution in the geologic environment. The long-term focus for this gap analysis is to be able to continuously and seamlessly upscale models from the atom scale to the geologic scale (Figure 3-7) as a means of addressing the ability to resolve processes at various time scales explicitly enough to have appropriate levels of confidence in the results of the models for the safety assessments. For the 
near term, working on the gaps identified below should provide the next step in accomplishing the long-term objectives.

i. Parametric upscaling from atomistic to continuum processes. There are two study areas that have been identified as useful pursuits for this purpose. The first study area is to perform explicit atomistic calculations of glass dissolution to reproduce both the rates and activation energies of glass dissolution reactions. This will be approached initially by modeling feldspar dissolution as a simplified case to serve as an idealized proxy for glass dissolution. This case has more structural definition in the solid phase and a smaller chemical system compared to glass wastes, but similar Si-Al structural limitations on dissolution rate control (see discussion in Section 3.3.5). In this case, delineating the appropriate level of reaction grouping is key to defining the point at which atomistic representations flange to the continuum methods. The second study area is to perform explicit atomistic models of silica diffusion in the aqueous solution away from the glass surface (within the gel layer) to begin constraining the transport parameters more directly, as these are generally used as fitting parameters in current continuum models. Reproducing silica diffusion explicitly would allow the physical properties of the gel layer (e.g., porosity, tortuosity) to be more fully evaluated from the progression in the experiments. (An adjunct to this study area would be to explicitly model both water and silica solid state diffusion within the glass itself.) Extraction of parameter values from experiments should occur in a framework that provides explicit process delineation both temporally and spatially. For upscaling purposes, parametric extraction for continuum processes from experiments should be performed with a narrow focus on the stage of the glass degradation experiment so that it can be accomplished in a manner consistent with the dominant rate limiting process(es).

ii. Definition of the equilibrium phase for glass dissolution and correlations among glass rate parameters. Because glass is a thermodynamically unstable phase, choosing the appropriate representative composition for defining the equilibrium condition for use in a chemical affinity approach to the dissolution rate has been handled in various empirical ways in the literature (e.g., Verney-Carron et al. 2010). This introduces a variability based on conceptual uncertainty into these approaches that may lead to disparate results that are not related directly to the use of chemical affinity to describe the rate, but simply due to the variability of the reference point assumed for the equilibrium condition. Most approaches have focused solely on the silica content of the glass (or the gel). Development of a more generally applicable equilibrium reference point that accounts for variable glass composition would provide a more coherent framework for upscaling studies (e.g., use a standard set of overall reaction products that represent the stable assemblage of the glass composition). Correlations between the rates of reactions and the free energy of overall reactions suggest this may be possible. Correlation development would also facilitate estimates of parameter values to be used for initial model construction and to delineate targeted experiments to measure confirmatory values.

iii. Aqueous composition evolution at the reactive interface. Once the degradation process has progressed to the Evolution Stage, the pore fluid in the gel layer that is in contact with the glass reactive interface is no longer the same as the bulk solution composition. The 
changes are a result of the reaction of the glass with the solution proximal to its surface and the transport processes occurring between the surface and the bulk solution outside the gel layers (note that this may be gradational on an atomistic scale such that a boundary region of a few to tens of monolayers of solution exists that is even more different from the local aqueous solution composition). A number of studies have considered the changes in dissolved silica but this should be expanded to include full water compositional evolution. Evaluating explicitly the changes at this location would be facilitated by a porescale reactive transport approach that included the major dissolved constituents.

iv. Surface area evolution of the reactive interface. In addition to the chemical changes that occur at the glass reactive interface as driven by gel layer development, physical occlusion of the reactive interface may reduce the overall mass of glass that is reacting with the fluids in the pores of the gel layer. Given that the porosity and density of this layer changes with time, this process could lead to changes in overall rate of glass degradation that are solely due to the physical mantling processes occluding fresh glass from further reaction. Explicit delineation of these processes would facilitate understanding of how to apply such changes over time in a repository setting and would aid in consistent parameter extraction from results of experiments. Evaluating explicitly these physical changes at the reactive interface would be facilitated by a continuum-scale reactive transport approach.

v. Field-scale progression of active surfaces contributing to the reactive interface total surface area using sub-continuum and continuum reformulations. In addition to changes in reactive or effective surface area at the glass-water interface due to an evolving gel layer structure, the effective surface area may increase dramatically as cracks in the glass surface nucleate and grow through time. Prior to being introduced to the repository setting, the nuclear waste glass forms may undergo expansion during heating due to radioactive decay. Subsequent cooling could generate cracks that propagate into the waste form structure. The formation of these cracks would not be accounted for through an analysis of changes in surface roughness at a single glass-water interface. At the continuum level, crack propagation theory is being modified to address non-linear material behavior at the tip of the crack (work by Fineberg et al. at Hebrew University, summarized in Grossman (2010)). At the sub-continuum scale, molecular modeling of cracks in silicon is demonstrating that the atomic-scale progress of a crack becomes hung up until atomic restructuring occurs and releases the crack tip to jump ahead (work by Buehler et al. at M.I.T., summarized in Grossman (2010)). Such advances in continuum and subcontinuum understanding present an opportunity for a coherent upscaling study for crack development.

Through analysis of fracture alteration in archeological glass and reaction-transport modeling, Verney-Carron et al. (2010) demonstrated that glass alteration at internal cracks will decrease over time, and that the majority of glass alteration occurs on external surfaces. It was concluded that the coupled glass alteration provided a negative feedback on the transport of constituents through the altering fracture pathways. The physical and chemical processes leading to decreased transport rate along altering cracks represent another area that may be amenable to upscaling analysis from sub-continuum to the continuum scale models. Additionally, developing general constraints on the integrated 
result of these field scale coupled processes (the occlusion of glass reactive interface surface area and additional crack growth) would address one of the major areas for upscaling from continuum models to field scale models. This is one of the key study areas for making the connection between high-fidelity continuum models and PA models that represent the system response at a higher level. Evaluating explicitly these integrated physical changes to the total glass surface area in the field would be facilitated by a continuum-scale reactive transport approach applied at the tens of centimeters to meter scale.

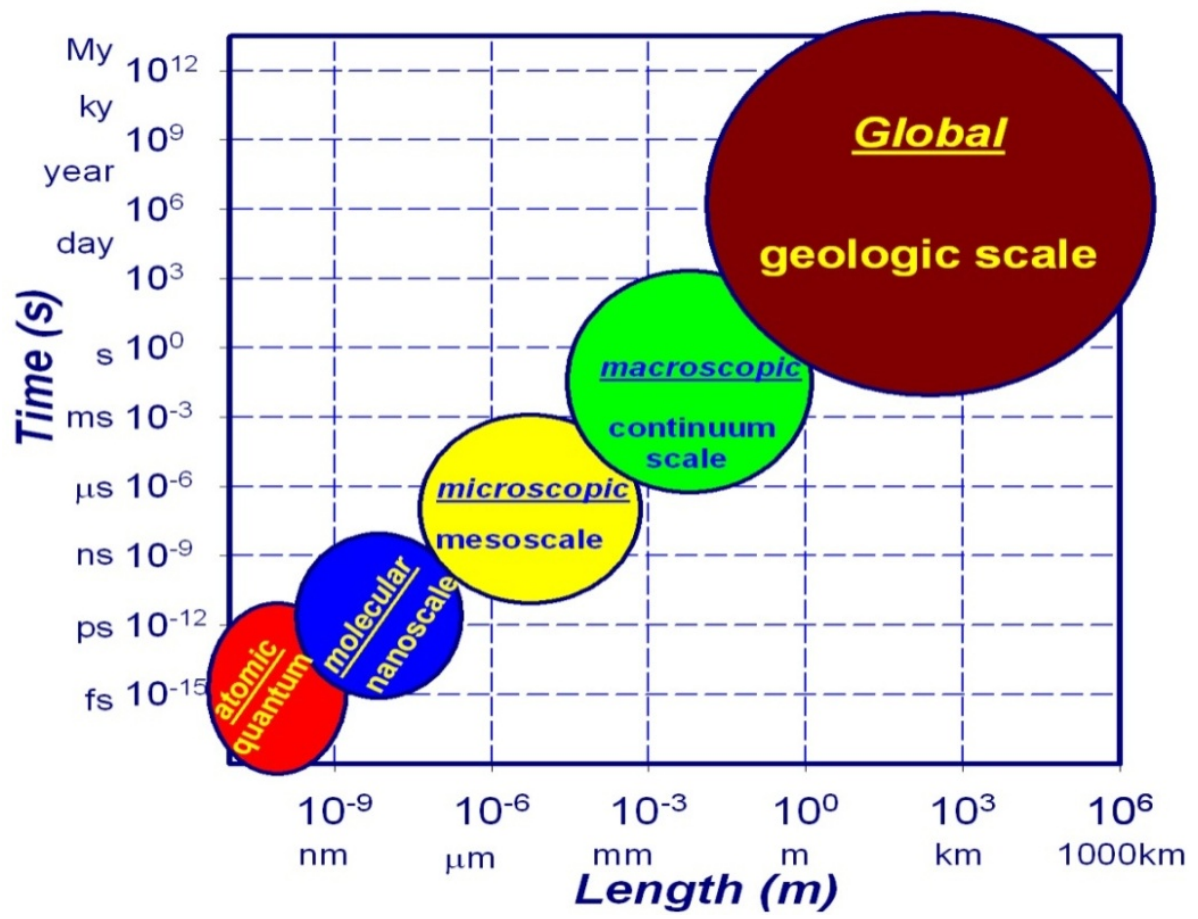

From Kalinichev (2004)

Figure 3-7. Time and length scales of geochemical modeling.

\subsection{Continuum-Scale Rate Models for Glass Dissolution}

\subsubsection{Overview of Kinetic Dissolution Rate Expressions}

Silicates are characterized by the formation of an alteration layer on the surface where the rate of Si release is less than the release of other cations. According to Brantley (2008), the formation of alteration layers on silicates has most commonly been attributed to simple leaching accompanied by surface condensation and reconstruction reactions. The dissolution is usually monitored by measuring the rate of increase of solute concentrations as a function of time (Brantley 2008). 
There are numerous kinetic rate laws for aluminosilicate dissolution that have been proposed in the literature. Six of these rate laws are described below to emphasize that these laws describe aluminosilicate dissolution using distinct parameter sets and still achieve partial success in describing observed dissolution data. This suggests that these rate laws include macroscopic parameters that are functions of micro- or nano-scale parameters that have not yet been determined.

For simple oxides, the dissolution rates are generally observed to be affected by the proton concentration of the reacting solution and are often described by the following empirical equation:

$$
r=k_{H} a_{H+}^{n}+k_{O H} a_{O H-}^{m}
$$

Where $k_{H}$ and $k_{O H}$ are the rate constants for proton- and hydroxyl-promoted dissolution respectively, $a_{i}$ is the activity of species $i$ in solution, and $n$ and $m$ are the partial orders of reaction (Brantley 2008).

A general kinetic rate equation for far-from-equilibrium dissolution of silicates containing cation $\mathrm{M}_{i}$ (Oelkers and Gislason 2001) has been proposed, assuming a precursor species controls dissolution:

$$
r=k \prod_{i=1, i \neq k}^{i}\left[\frac{K_{i}\left(\frac{a_{H^{+}}^{v_{i}}}{a_{M_{i}}^{v_{i}}}\right)^{S}}{\left(1+K_{i}\left(\frac{a_{H^{+}}^{v_{i}}}{a_{M_{i}}^{v_{i}}}\right)^{S}\right)}\right]
$$

Here $k$ is the rate constant, $\mathrm{K}_{i}$ is the equilibrium constant describing the exchange reaction between protons and the metal cation $\mathrm{M}_{i}$ at the surface that forms the precursor species, and the parameters $v_{i}$ and $s$ are stoichiometric coefficients for species appearing in the formation reaction for the precursor in the rate-limiting step.

Quartz dissolution has been expressed by the following rate equation:

$$
r=k_{S i O H}(T) \theta_{\equiv S i O H}+k_{S i O^{-}}(T) \theta_{\equiv S i O_{t o t}^{-}}
$$

where each rate constant describes the reaction at a given surface site as indicated, $\theta_{\equiv S i O H}$ is the fraction of total neutral surface sites, and $\theta_{\equiv \text { SiO }_{\text {tot }}^{-}}$is the sum of the fractions of total sites existing as deprotonated surface hydroxyls and as sites with adsorbed $\mathrm{Na}+\left(\theta_{\equiv \operatorname{SiONa}}{ }^{+}\right)$. This equation successfully describes quartz dissolution from 25 to $300^{\circ} \mathrm{C}$ for $\mathrm{pH} 2-12$ at variable ionic strengths (Dove 1994; 1995).

Net rates of dissolution for amorphous silica into solutions with and without $\mathrm{NaCl}$ have been described successfully using the following model (Icenhower and Dove 2000): 


$$
r=k_{+}\left(a_{S O_{2(a q)}}\right)\left(a_{H 2 O}\right)^{2}(1-\Omega)
$$

where $\mathrm{k}_{+}$is the dissolution rate and $\Omega=\exp (\Delta \mathrm{G} / \mathrm{RT})$. This phase dissolves at a rate approximately ten times faster than quartz under comparable conditions.

Extrapolation of the rate constant for dissolution to other temperatures can be accomplished with the Arrhenius equation:

$$
k_{H}(T)=k_{H}\left(T_{o}\right) e^{-\left(E_{a} / R T\right)}
$$

where $k_{H}\left(T_{o}\right)$ (mol mineral $\mathrm{m}^{-2} \mathrm{~s}^{-1}$ ) is the temperature-independent pre-exponential factor, $\mathrm{E}_{\mathrm{a}}$ is the apparent activation energy, $\mathrm{R}$ is the gas constant, and $\mathrm{T}$ is the absolute temperature.

Transition state theory (TST) is generally considered to be appropriate only for elementary reactions. However, if a complex mechanism is rate-limited by a single elementary reaction, then TST may be usefully applied to the overall reaction. TST suggests that the forward rate minus the reverse rate $\left(r_{n e t}\right)$ is linear with respect to $\Delta \mathrm{G}$ near equilibrium according to the following equation:

$$
r_{n e t}=-k_{+} \prod a_{j}^{m_{j}}\left(1-\exp \left(\frac{n_{i} \Delta G}{R T}\right)\right)
$$

Here $k_{+}$is the rate constant for the forward rate, $a_{j}$ is the activity of species $j$ in the ratedetermining reaction, $m_{j}$ and $n_{i}$ are constants, $\mathrm{R}$ is the gas constant, and $\mathrm{T}$ is the absolute temperature. In some treatments, the $\Delta \mathrm{G}$ term is also divided by Temkin's average stoichiometric number, a constant that refers to the relative rate of destruction of the activated complex divided by the overall dissolution rate (Oelkers et al. 1994; Oelkers 2001).

Several investigators have approached crystal dissolution from the perspective that it can be represented as the inverse of crystal growth (e.g., Dove et al. 2005) or described via the combination of specific surface structural mechanisms (e.g., Lasaga and Luttge 2001). Lasaga and Luttge (2001) introduced a model for dissolution based on the propagation of stepwaves from etch pits. Experimental data (vertical scanning interferometry (VSI)) suggested that although etch pits form, the entire crystal surface dissolves. Observations suggest that stepwaves emanating from etch pits combine to lower the entire crystal surface. The evolution of stepwaves can be quantified with this equation:

$$
v(r)=v_{\text {step }}\left(1-\frac{1-e^{-\frac{\sigma v}{r k T} e^{\frac{u(r) v}{k T}}}}{1-e^{\frac{\Delta G}{k T}}}\right)
$$

where $v_{\text {step }}$ is the velocity of a series of straight steps, $v(r)$ is the velocity of dissolution stepwaves at a distance $r$ from the dislocation defect, $u(r)$ is the strain field of dislocation defects, $v$ is the molecular volume, $k$ is the Boltzmann's constant, $\mathrm{r}$ is the radius of a circular dissolution step, $\sigma$ is the surface free energy. 
The overall dissolution rate is written as:

$$
\text { Rate }=v_{\infty}\left(1-e^{\Delta G / k T}\right) \tanh \left[\frac{1}{2 x_{s} f\left(r_{p i t}\right)}\right] f\left(r_{p i t}\right)
$$

where the modifying function $f\left(r_{\text {pit }}\right)$ comes from Equation 3.2.7 above, and $x_{s}$ is a surface diffusion distance expressed in molecular units. Using this expression, the dissolution rate approaches a simple linear rate or TST-like equation far from equilibrium, and exhibits a nonlinear decrease near equilibrium (Lasaga and Luttge 2003). This equation can be tested with detailed kinetic models such as Monte Carlo methods (see Section 3.3). In these methods, $\sigma$, $u(r)$, and $x_{s}$ are known exactly. The only adjustable parameter is the dissolution rate at infinite dilution (i.e., the far from equilibrium "dissolution plateau").

\subsubsection{Quantification of Rate Law Parameters}

One critical issue in the development and use of kinetic rate laws is how to quantify the surface area involved in crystal-fluid interaction. The overall dissolution rate is often defined as a function of the difference in Gibbs free energy between solid and its dissolved molecules $(\Delta G)$, and the surface area $(A)$ that participates in the dissolution process.

There are three ways in which the surface area is described: (1) geometric, (2) total, and (3) reactive. This last term is equivalent to the reactive interface surface area described above. The geometric surface area ignores surface topography or roughness and tends to underestimate total surface area. The total surface area includes surface roughness. One approach to measuring the total surface area is the Brunauer, Edward and Teller (BET) method which measures the amount of an inert gas adsorbed on a sample under controlled conditions at a specific temperature. A key assumption is that the gas adsorbs in a monomolecular layer on the sample surface. In practice, the method works well for relatively large surface areas (1-1000 $\mathrm{m}^{2} / \mathrm{g}$ or larger). With krypton gas, smaller surface areas $\left(>0.1 \mathrm{~m}^{2} / \mathrm{g}\right)$ can be revealed as well as nanometer-scale porosity. The concept of reactive surface area is that some sections of a surface are more reactive than others (Luttge and Arvidson 2008). External and internal surface area have been defined as that portion over which a fluid is free-flowing and that portion that is not in contact with the fluid (pore) or only in contact with the fluid under stagnant conditions.

Several analytical techniques are used to quantify surface topography including atomic force microscopy (AFM), interferometry, and electron microscopy. AFM is a valuable high-resolution imaging technique that has the ability to track the motion of monomolecular steps in situ, as a function of solution composition in a flow-through environment (Luttge and Arvidson 2008). Interferometry provides surface topography information over the $\mathrm{nm}-\mathrm{cm}$ scale. Luttge et al. (1999; 2003) and Luttge (2004) introduced vertical scanning interferometry and its application to mineral dissolution and growth. Vertical resolution for this technique is on the order of $\AA-\mathrm{nm}$ scale. Measurements of surface-normal height changes can be converted directly into dissolution or growth rates and averaged over the entire solid surface. Scanning electron microscopy (SEM) produces images of a sample surface that resolve surface features and microtopography. Environmental SEM circumvents the need for a conductive coating and a high vacuum. Surface chemistry is also studied by X-ray photoelectron spectroscopy (XPS), secondary ion mass spectrometry (SIMS), and X-ray (grazing incidence) diffraction to analyze the elemental 
composition of the surface and near surface layers, the oxidation state of these elements, and the composition of surface features relative to the bulk crystal.

\subsection{Molecular-Level Studies of Dissolution}

From Section 3.2, it is evident that many different parameters may contribute to glass dissolution and degradation rates including different surface site protonation states at different $\mathrm{pH}$ values, the chemical activities of aqueous species, and changes in the reactive interface surface area and interfacial phases (i.e. gel layers) over time. Interpreting glass degradation rates using leaching experiments alone is challenging because the molecular-level mechanisms involved in glass dissolution occur at the glass-water interface, which can only be fully characterized at smaller scales. As a consequence, much computational and analytical research has been conducted to determine molecular-level reaction mechanisms at this interface.

More research has been done to incorporate atomistic data into continuum models for silicate and aluminosilicate minerals than for glasses, because of a strong desire by the geochemical community to understand silicate weathering in the natural environment. For over twenty years, geochemists have recognized that reactions occurring at the mineral-water or rock-water interfaces are critical to field scale processes (Hochella and White 1990). Although simulating the degradation of glass nuclear waste forms is even more challenging, in part because of the larger chemical system of glass, many of the key uncertainties/challenges are comparable to those for aluminosilicate minerals. Some of the key challenges in modeling and upscaling atomistic data into continuum models for aluminosilicate dissolution include: (1) determining surface site hydroxylation and charge; (2) calculating activation energies for elementary reactions; (3) relating the "apparent activation energy" from leaching experiments to the calculated activation energies for elementary reactions; (4) confirming proposed reaction paths; (5) modeling the formation of gel layers and changes in gel layer thickness with variations in mineral or glass composition; (6) modeling transport of aqueous species through the gel layer; and (7) identifying changes in reactive surface area through time due to changes in the gel layer and fracturing of the dissolving material. The following subsections review the most recent modeling research in aluminosilicate dissolution and upscaling in order to establish a baseline for future work and identify upscaling gaps and uncertainties.

\subsubsection{Determination of Reaction Mechanisms}

Dissolution rates of highly soluble and highly insoluble material tend to be relatively fast and slow, respectively. Dissolution of highly soluble material has a greater likelihood of becoming transport-limited and the dissolution of low-solubility materials is likely to be interface-limited. Transport or interface limited dissolution can be inferred based upon the morphology of the dissolving surface. In general, dissolution can be conceptualized as the sum of (1) horizontal movement of atomic-scale steps defining incomplete surface layers (step retreat), and (2) removal of atoms by $2 \mathrm{D}$ or pit nucleation in the material. Etch pits nucleate at both perfect surface sites and at defect outcrops. Regardless of the type of pit, when dissolution occurs near equilibrium, pits are unlikely to form. From small to large undersaturations, dissolution steps are more likely to nucleate at crystal edges, at dislocation etch pits, at impurity etch pits, at point defects, and at perfect surfaces respectively because both the rates of reaction at these sites differ 
and the relative number of these sites varies as a function of undersaturation. These conceptual models for dissolution are outgrowths of classical crystal growth (Burton-Cabrera-Frank) theory (Dove et al. 2005).

The preferential leaching of elements from a dissolving surface is driven by differences in site energies for cations in the material structure. For dissolving silicates, non-stoichiometric dissolution is especially pronounced at lower $\mathrm{pH}$ and can be detected using surface-sensitive spectroscopies such as XPS or SIMS. Gel layers that form on feldspar surfaces demonstrate the characteristics of amorphous silica when analyzed after dissolution. The development of a leached layer on dissolving feldspar surfaces is influenced by the $\mathrm{Al} / \mathrm{Si}$ ratio of the feldspar. The mechanism underlying such observations has been investigated using computational techniques. For example, $a b$ initio calculations of the optimized geometries of dissolving surface clusters have been calculated. As the $\mathrm{Al} / \mathrm{Si}$ ratio is increased in the cluster, the average bond length of $\mathrm{Si}-\mathrm{O}$ bonds within Al-O-Si linkages increases from 1.58 to $1.62 \AA$ suggesting that hydrolysis of Al-O-Si bonds become easier. These theoretical results can partially explain why the dissolution rates of plagioclase feldspar increases with the $\mathrm{Al} / \mathrm{Si}$ ratio, and why preferential leaching of $\mathrm{Al}$ occurs more readily at high $\mathrm{Al} / \mathrm{Si}$ ratio. However, alteration layers on feldspars such as labradorite $(\mathrm{Al} / \mathrm{Si}=0.66-0.8)$ have also been attributed to solution-reprecipitation (Hellmann et al. 2003).

Transport-controlled dissolution can either refer to slow transport through the fluid boundary layer or through an alteration layer on the material surface. Such alteration layers grow on many dissolving silicates where the rate of $\mathrm{Si}$ release is less than release of other cations. The mechanism of formation of alteration layers on silicates has been attributed to simple leaching accompanied by surface condensation and reconstruction reactions (Casey and Ludwig 1996; Hellmann et al. 2003). For example, the presence of six-coordinate Al, observed on a partially dissolved albite surface was attributed to reconstructive reactions occurring within a layer formed by leaching (Tsomaia et al. 2003). However, in cases such as labradorite dissolved at low $\mathrm{pH}$, the sharp interface between surface layer and bulk mineral may be evidence of dissolution, reprecipitation, and reconstruction (Hellmann et al. 2003). According to this solution-precipitation hypothesis, reprecipitation reactions occur well below solubility limits for secondary phases. In either case, the hydrolysis of bridging oxygens generally reduces the connectedness of network atoms before the atom is released into solution (Hellmann et al. 1990; Brantley and Stillings 1997; Hamilton et al. 2001; Tsomaia et al. 2003). A distribution of tetrahedral or $\mathrm{Q}^{\mathrm{i}}$ sites (where $\mathrm{i}=4,3,2$, or 1 bridging oxygen) must characterize the gel layer regardless of whether it forms by solution-precipitation or by leaching.

\subsubsection{Quantum Mechanics Cluster Calculations}

Quantum mechanics calculations have been used to study the kinetics of silicate and aluminosilicate dissolution for twenty years (Casey et al. 1990; Xiao and Lasaga 1994; 1996; Kubicki et al. 1997; Criscenti et al. 2005). These calculations are performed on small clusters of atoms, that can be representative of either glass or crystal structures. Therefore, the results of these calculations can be applied to the dissolution of both natural minerals such as quartz and feldspar and silica-based glasses. In general, the proposed steps for breaking a Si-O-Si bond include $\mathrm{H}^{+}$transfer to a bridging oxygen atom followed by the formation of a stable 5-fold $\mathrm{Si}$ 
intermediate. Quantum mechanics is used to calculate activation energies associated with different proposed elementary steps along a reaction path. It should be noted that the dissolution of one formula unit of albite will require a series of up to 10 elementary steps including $1 \mathrm{Na}^{+} / \mathrm{H}^{+}$ exchange, the hydrolysis of 3 Al-O-Si linkages and 6 Si-O-Si linkages (Kubicki 2008). The framework of aluminosilicate glasses and the feldspar minerals consists of linked $\mathrm{SiO}_{4}$ and $\mathrm{AlO}_{4}$ tetrahedra. At the solid surface, a $\mathrm{Si}$ atom may be bonded to three bridging $\mathrm{O}$ atoms $\left(\mathrm{Q}^{3} \mathrm{Si}\right)$, two $\mathrm{O}$ atoms $\left(\mathrm{Q}^{2} \mathrm{Si}\right)$ or one $\mathrm{O}$ atom $\left(\mathrm{Q}^{1} \mathrm{Si}\right)$ of the glass or mineral structure. It has been suggested (e.g., Brantley 2004; Criscenti et al. 2006) that the calculated activation energies for breaking the different types of $\mathrm{Si}(\mathrm{Al})-\mathrm{O}-\mathrm{Si}(\mathrm{Al})$ bonds can be compared to the activation energies determined through fitting rate expressions to bulk dissolution data, to infer which reaction is the ratelimiting step. However, it has also been proposed (e.g., Zhang and Luttge 2008) that the apparent activation energy determined from a bulk dissolution experiment is a function of numerous activation energies associated with many ongoing reactions. These concepts will be discussed further below.

Criscenti et al. (2005; 2006) modeled reaction pathways for hydrolyzing Si-O-Si and Si-O-Al using model clusters of a central $\mathrm{Si}$ or Al tetrahedron surrounded by three other Si tetrahedra $\left(\mathrm{Q}^{3} \mathrm{Si}\right.$ or $\left.\mathrm{Q}^{3} \mathrm{Al}\right)$. The calculations for $\mathrm{Q}^{3} \mathrm{Si}$ showed that nearby $\mathrm{H}_{2} \mathrm{O}$ molecules play roles in $\mathrm{H}^{+}$ transfer both to the bridging oxygen atom in the Si-O-Si linkage to form $\mathrm{Si}-(\mathrm{OH})-\mathrm{Si}$ and back to a $\mathrm{H}_{2} \mathrm{O}$ molecule to reform $\mathrm{H}_{3} \mathrm{O}^{+}$. These solvation effects were neglected in earlier, smaller cluster studies of these systems (Xiao and Lasaga 1994; 1996). Solvation effects can impact the stability of various species (Kubicki 2001) and the calculated activation energy barriers. Pelmenschikov et al. (2000) also used molecular orbital-transition state theory (MO-TST) to examine the hydrolysis of $\beta$-cristobalite $\left(\mathrm{SiO}_{2}\right)$. They chose to constrain the Si-O-Si bond lengths and angles according to the $\beta$-cristobalite structure, while Criscenti et al. (2006) allowed for full relaxation of the silica cluster. Pelmenschikov et al.'s approach resulted in higher calculated activation energies for $\mathrm{Si}-\mathrm{O}-\mathrm{Si}$ bond breakage.

A recent example of the application of the MO-TST approach to isotope exchange kinetics can be found in Felipe et al. (2003). The summary provided here comes from Kubicki (2008). The exchange of $\mathrm{O}$ atoms in orthosilicate acid, $\mathrm{Si}(\mathrm{OH})_{4}$, with $\mathrm{H}_{2} \mathrm{O}$ molecules was followed to predict values of the fractionation factor and exchange rate. The predicted rate of exchange was rapid, consistent with the common assumption for this reaction in natural waters. A key to the success of the Felipe et al. (2003) study was the inclusion of multiple $\mathrm{H}_{2} \mathrm{O}$ molecules in the model. Previously, a single $\mathrm{H}_{2} \mathrm{O}$ molecule had been included to examine silica- $\mathrm{H}_{2} \mathrm{O}$ interactions in the interest of computational efficiency. By performing simulations using 3 and $7 \mathrm{H}_{2} \mathrm{O}$ molecules reacting with $\mathrm{Si}(\mathrm{OH})_{4}$, Felipe et al. (2003) could examine cooperative effects among the $\mathrm{H}_{2} \mathrm{O}$ molecules that are critical to the $\mathrm{H}^{+}$-transfer process. Model calculations that neglect this possibility will most likely be in error no matter what level of theory is used to calculate structures and energies. Several methods for calculating the structures and energies of test configurations were performed in order to estimate possible error within the model system. The formation of the five-coordinate Si complex was predicted as suggested in a number of earlier modeling studies of silica-water interactions. One difference from previous is that instead of a $(\mathrm{OH})_{4} \mathrm{SiOH}_{2}$ complex, a $\left[\mathrm{Si}(\mathrm{OH})_{5}\right]^{1-}$ complex was predicted with an $\mathrm{H}^{+}$transfer occurring from the incoming $\mathrm{H}_{2} \mathrm{O}$ molecule to a nearby $\mathrm{H}_{2} \mathrm{O}$ to form an $\mathrm{H}_{3} \mathrm{O}^{+}$. In addition, the $\mathrm{H}_{3} \mathrm{O}^{+}$formed a 
dimer with another $\mathrm{H}_{2} \mathrm{O}$ molecule facilitating the cooperative transfer of the proton to the leaving $\mathrm{OH}^{-}$on the $\left[\mathrm{Si}(\mathrm{OH})_{5}\right]^{1-}$ complex to result in the exchanged $\mathrm{Si}(\mathrm{OH})_{4}$ molecule.

Nangia and Garrison (2008) and Morrow et al. (2009) calculated the activation energy for the hydrolysis of Si-O and Al-O bonds from quantum mechanical calculations on small silicate and aluminosilicate clusters, using a constrained optimization approach similar to Xiao and Lasaga (1994; 1996) and Criscenti et al. (2005; 2006). Nangia and Garrison (2008) examined the hydrolysis of Si-O-Si bonds for protonated, neutral and deprotonated silica clusters according to the following reactions:

$$
\begin{aligned}
& (\mathrm{OH})_{3} \mathrm{Si}-\mathrm{O}-\mathrm{Si}(\mathrm{OH})_{3} \mathrm{H}^{+}+\mathrm{H}_{2} \mathrm{O} \rightarrow(\mathrm{OH})_{3} \mathrm{Si}-\mathrm{OH}+\mathrm{Si}(\mathrm{OH})_{3}-\mathrm{OH}_{2}^{+} \\
& (\mathrm{OH})_{3} \mathrm{Si}-\mathrm{O}-\mathrm{Si}(\mathrm{OH})_{3}+\mathrm{H}_{2} \mathrm{O} \rightarrow(\mathrm{OH})_{3} \mathrm{Si}-\mathrm{OH}+(\mathrm{OH})_{3} \mathrm{Si}-\mathrm{OH} \\
& (\mathrm{OH})_{3} \mathrm{Si}-\mathrm{O}-\mathrm{Si}(\mathrm{OH})_{2} \mathrm{O}^{-}+\mathrm{H}_{2} \mathrm{O} \rightarrow(\mathrm{OH})_{3} \mathrm{Si}-\mathrm{OH}+(\mathrm{OH})_{3} \mathrm{Si}^{-} \mathrm{O}^{-}
\end{aligned}
$$

Figure 3-8 (top diagram) illustrates the reaction path for the protonated cluster. The dissolution process at a protonated surface site proceeds through two transition states (TS1 and TS2), and a stable penta-coordinated Si intermediate (I). In contrast, the hydrolysis of the $\mathrm{Si}-\mathrm{O}_{\mathrm{br}}-\mathrm{Si}$ bond in the neutral species (Figure 3-8 middle diagram) has only one transition state. The reaction profile for the deprotonated species (Figure 3-8 bottom diagram) is similar to the profile for the protonated cluster. For both the protonated and deprotonated systems, the activation energy barrier associated with the formation of the penta-coordinated silica intermediate is larger than the energy involved in breaking the $\mathrm{Si}-\mathrm{O}_{\mathrm{br}}$ bond, suggesting that this is the rate-limiting step in the reaction. The neutral reaction is a one-step process with concerted bond-breaking and forming processes in the transition state. The energy barrier for the neutral reaction is higher than in the rate-limiting steps for the dissolution of the protonated and deprotonated species. These results are consistent with the observation that silica dissolution occurs more quickly in acidic and alkaline solutions than in neutral waters. 

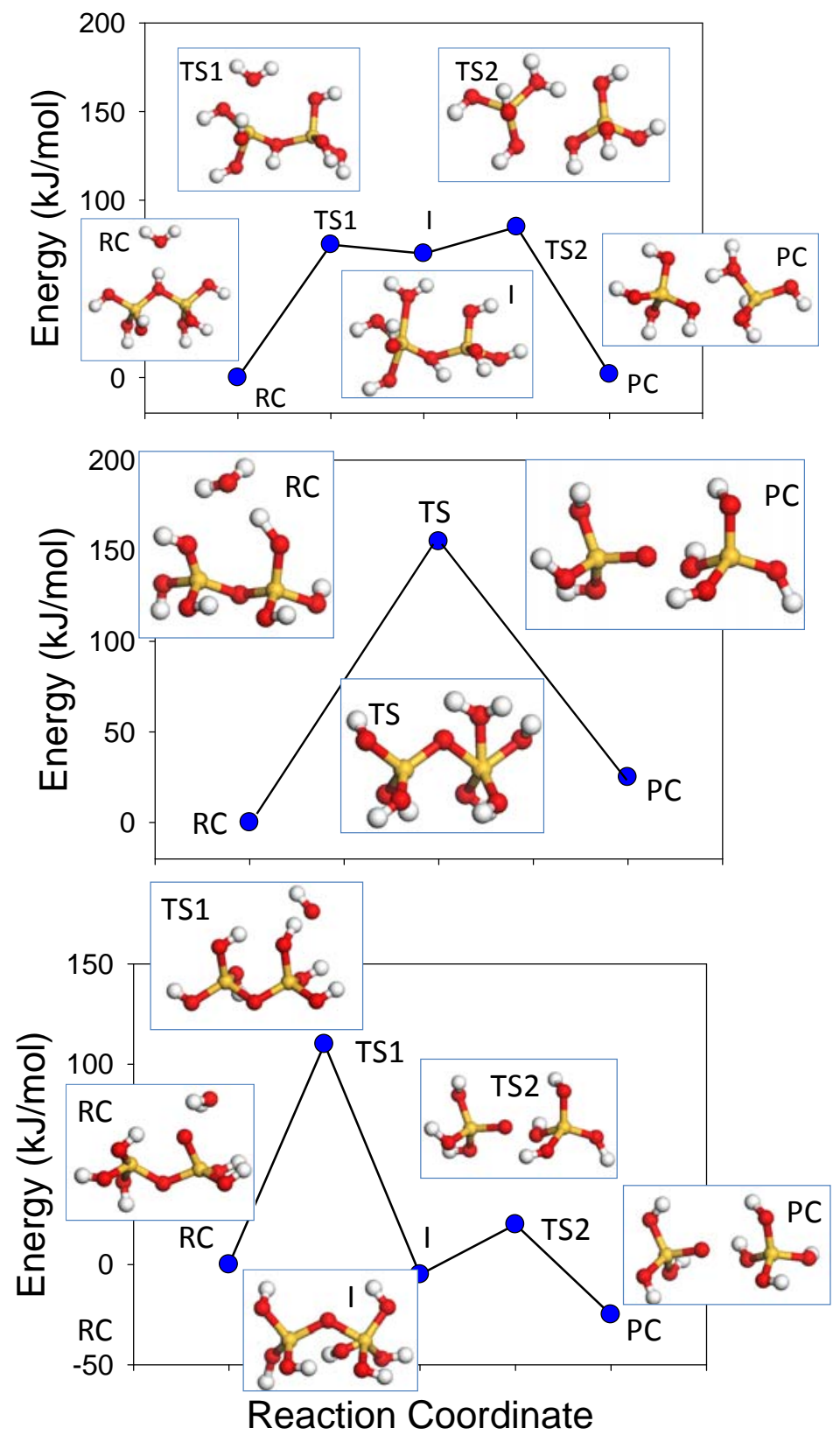

The geometries of the reactant complex (RC), first transition state (TS1), intermediate (I), second transition state (TS2), and the product complex (PC) are shown along the path. The Si, O, and $\mathrm{H}$ atoms are shown in light brown, red, and white colors, respectively. (after Nangia and Garrison 2008).

Figure 3-8. Energy profile $(\mathrm{kJ} / \mathrm{mol})$ of the Si-O-Si hydrolysis reaction along the reaction coordinates for the protonated, neutral, and deprotonated species. 
Morrow et al. (2009) performed similar calculations to investigate the hydrolysis of both $\mathrm{Si}-\mathrm{O}_{\mathrm{br}}{ }^{-}$ $\mathrm{Al}$ and $\mathrm{Al}-\mathrm{O}_{\mathrm{br}} \mathrm{Si}$, where in the first case the $\mathrm{Si}-\mathrm{O}_{\mathrm{br}}$ bond is broken, and in the second case, the $\mathrm{Al}-\mathrm{O}_{\mathrm{br}}$ bond is broken. For $\mathrm{Si}-\mathrm{O}_{\mathrm{br}}$ hydrolysis, the reaction profiles are similar to those reported for $\mathrm{Si}-\mathrm{O}_{\mathrm{br}}-\mathrm{Si}$ by Nangia and Garrison (2008). A major difference occurs for the deprotonated site reaction; for $\mathrm{Si}-\mathrm{O}_{\mathrm{br}}-\mathrm{Al}$, the activation energy barrier for breaking the $\mathrm{Si}-\mathrm{O}_{\mathrm{br}}$ bond is higher than the barrier to forming a penta-coordinated $\mathrm{Si}$, while the opposite was found for $\mathrm{Si}-\mathrm{O}_{\mathrm{br}} \mathrm{Si}$.

The description of an $\mathrm{Al}-\mathrm{O}_{\mathrm{br}}$-Si site on the surface is more complicated. $\mathrm{Al}$ is known to assume different coordination states according to $\mathrm{pH}$. In low $\mathrm{pH}$ ranges, $\mathrm{Al}$ is hexa-coordinated in solution, therefore a hexa-coordinated $\mathrm{Al}-\mathrm{O}_{\mathrm{br}}-\mathrm{Si}$ site with a protonated $\mathrm{O}_{\mathrm{br}}$ is used to represent a protonated surface site. Aluminum can be penta-coordinated in slightly acidic to neutral conditions, and is tetra-coordinated in basic $\mathrm{pH}$ ranges. Schematics of the three $\mathrm{Al}-\mathrm{O}_{\mathrm{br}}-\mathrm{Si}$ sites are depicted in Figure 3-9. Despite the potential for Al to be present in three different coordination states, the reaction profiles for the hydrolysis of $\mathrm{Al}-\mathrm{O}_{\mathrm{br}}-\mathrm{Si}$ in protonated, neutral, and deprotonated states all proceed through one-step mechanisms. In addition, the activation energy barriers for breaking the Al- $\mathrm{O}_{\mathrm{br}}$ bonds are lower than those for breaking $\mathrm{Si}-\mathrm{O}_{\mathrm{br}}$ bonds.
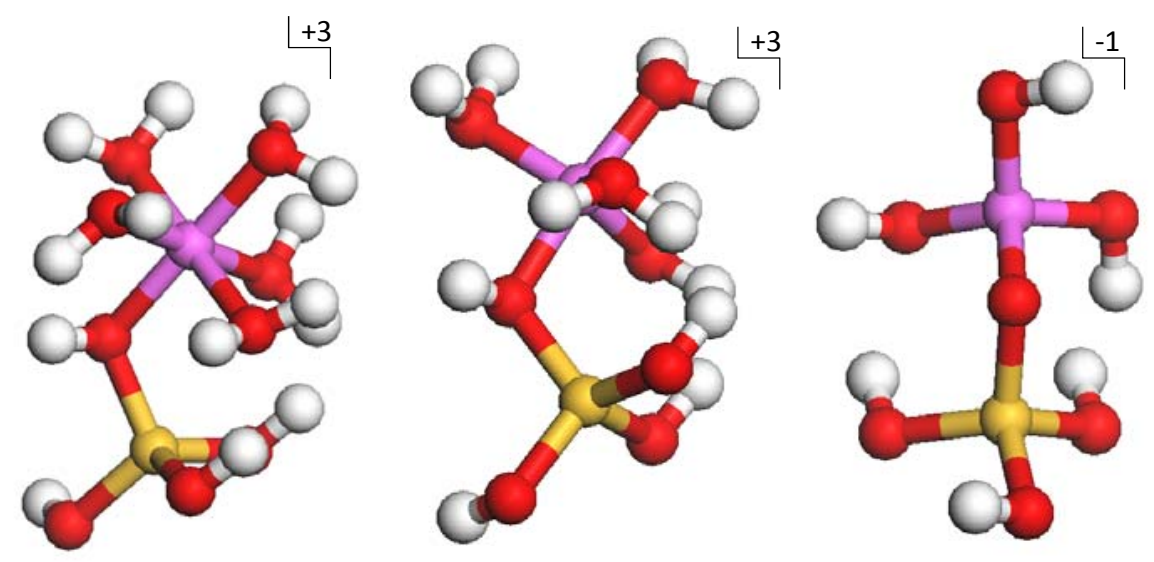

Atoms color coded as: $\mathrm{Al}$ - purple; $\mathrm{Si}$ - yellow; $\mathrm{O}$ - red; $\mathrm{H}$ - white. The net charge on the cluster is shown on the top right of each structure (after Morrow et al. 2009)

Figure 3-9. Schematic of the Al- $\mathrm{O}_{\mathrm{br}}-\mathrm{Si}$ surface site in (a) protonated, (b) neutral, and (c) deprotonated states.

Nangia and Garrison (2008) calculated rate constants $\left(\mathrm{s}^{-1}\right) k_{\equiv S i O H_{2}^{+}}, k_{\equiv S i O H}$, and $k_{\equiv S i O^{-}}$from the optimized structures of the reactants, products and transition states for the hydrolysis of protonated, neutral and deprotonated Si-O-Si species using TST. The TST rate constant is:

$$
k^{\ddagger}(T)=\frac{k_{B} T}{h} \frac{Q^{\ddagger}(T)}{Q(T)} e^{-V^{\ddagger} / k_{B} T}
$$

where $V^{\ddagger}$ is the barrier height of the reaction, $Q^{\ddagger}$ is the partition function of the transition state, $\mathrm{Q}$ is the partition function of the reactants, $k_{B}$ is Boltzmann's constant, $\mathrm{h}$ is Planck's constant and $T$ is the temperature. 
The dissolution rates $\left(\mathrm{mol} \mathrm{m} \mathrm{m}^{-2}\right.$ ) are calculated for the 2-12 $\mathrm{pH}$ range at $298 \mathrm{~K}$, using the following equation:

$$
r=k_{\equiv \mathrm{SiOH}_{2}^{+}} \theta_{\equiv \mathrm{SiOH}_{2}^{+}} \rho+k_{\equiv \mathrm{SiOH}} \theta_{\equiv \mathrm{SiOH}} \rho+k_{\equiv \mathrm{SiO}^{-}} \theta_{\equiv \mathrm{SiO}^{-}} \rho
$$

where $\theta_{\equiv \mathrm{SiOH}_{2}^{+}}, \theta_{\equiv \mathrm{SiOH}}$, and $\theta_{\equiv \mathrm{SiO}^{-}}$are the fraction of the protonated surface sites, and $\rho$ is the molar surface density of reactive sites. For quartz, the total number of reactive surface sites (or surface hydroxyl groups) is $5-7 \mathrm{~nm}^{-2}$. This rate expression (Equation 3.3.5) does not distinguish the surface sites based on connectedness of the tetrahedrally coordinated silicon atoms (i.e., $\mathrm{Q}^{1}$, $\left.\mathrm{Q}^{2}, \mathrm{Q}^{3}\right)$ on the surface. These reaction rates were compared to the experimental results of Dove and Elston (1992), Knauss and Wolery (1988), Schwartzentruber et al. (1987), and Wollast and Chou (1988). The fractions of protonated, neutral, and deprotonated sites were determined based on the work of Hayes and Leckie (1987). While the comparison was reasonably successful, the fractions of charged and neutral sites originate from continuum model fits. Therefore these results cannot be attributed to using strictly atomistic information.

$A b$ initio cluster calculations for nuclear waste glass dissolution have been proposed by Aertsens and Ghaleb (2001) to provide (1) a better understanding and modeling of effects of solution composition (including $\mathrm{pH}$ ) on the glass dissolution rate, (2) more accurate estimates of the activation energy for glass dissolution (values in the literature diverge considerably), or (3) a better understanding of the mixed alkali effect. Another application may be in development of waste packages - determining what to put in the waste package to decrease dissolution rates. Concerning the link with other modeling methods, $a b$ initio methods can provide (1) the potential energy functions used in molecular dynamics (MD) simulations, or (2) the activation energies or equilibrium constants needed for Monte Carlo (MC) modeling, or (3) equilibrium constants that can be compared with those in geochemical databases.

Aertsens and Ghaleb (2001) also point out that the structure of the gel that forms between the dissolving glass and aqueous solution can be studied through $a b$ initio calculations. The pores in the gel may be considered as the hole of a ring formed by glass formers. Tossell and SaghiSzabo (1997) performed $a b$ initio calculations for such rings formed by four tetrahedrally coordinated atoms connected by bridging oxygens. The results show that $\mathrm{Si}-\mathrm{Al}-\mathrm{Si}-\mathrm{Al}$ was more stable than $\mathrm{Si}-\mathrm{Si}-\mathrm{Al}-\mathrm{Al}$. However, the incorporation of a proton or $\mathrm{Ca}^{2+}$ made the $\mathrm{Si}-$ $\mathrm{Si}$ - Al - Al configuration more stable. Similar calculations were executed for boron instead of aluminum. For the dissolution of $\mathrm{Na}^{+}$-aluminosilicate glasses, their calculations show that the reaction of $\mathrm{H}_{2} \mathrm{O}$ with $\mathrm{Si}_{2} \mathrm{Al}_{2} \mathrm{O}_{12} \mathrm{Na}_{2}$ (which can be considered as a simulation of ion exchange) does not lead to the hydrolysis of the Si-O-Al bond. Instead, the water molecule sits near the $\mathrm{Na}^{+}$.

\subsubsection{Differences, Gaps, and Uncertainties}

Several different approaches are used in the MO-TST modeling of aluminosilicate clusters:

(1) Typically, small cluster calculations are performed unconstrained, allowing the cluster to reach a global minimum. However, the activation energies calculated by Pelmenschikov 
et al. (2000) are clearly higher because they constrained the calculations to the material structure. If the lower activation energies are used for upscaling, then predicted dissolution rates will be faster than if the activation energies from unrelaxed clusters are used. The use of lower activation energies might be regarded as "conservative" from a risk assessment perspective.

(2) The inclusion of several explicit water molecules in MO-TST calculations can change the reaction path, and the charge and structure of both the transition state and stable intermediates in the hydrolysis of aluminosilicate clusters. It is not clear how these differences will manifest themselves in calculated activation energies and upscaled system properties.

(3) Quantum mechanics calculations can be used to look at the energetic of repolymerization on a surface. Pelmenschikov et al. (2001) looked at the impact of self-healing on silica surfaces, but this has not been investigated for aluminosilicate or borosilicate glasses.

(4) Nangia and Garrison (2008) used the density of protonated and deprotonated quartz surface sites derived from bulk surface titration data and the use of a continuum surface complexation model to determine dissolution rates over a $\mathrm{pH}$ range of 2-12. While this is a sensible approach, strict "upscaling" would require that the density of protonated and deprotonated surface sites also be derived from molecular-scale data or calculations.

Based on these observations, two areas for further analyses have been identified as primary gaps.

i. Calculate the protonation and deprotonation of different types of surface sites on glass surfaces using $a b$ initio molecular dynamics calculations. Ab initio MD calculations have been used to calculate the $\mathrm{pK}_{\mathrm{a}}$ 's for different quartz surface sites (Leung et al. 2009). The calculated results were interpreted by comparison to surface titration data over a $\mathrm{pH}$ range collected using second harmonic generation spectroscopy (Ong et al. 1992). Similar methods could be used to investigate the protonation and deprotonation of different types of surface sites on glass surfaces. These $a b$ initio MD calculations would need to be performed in combination with potentiometric titrations, vibrational and nuclear magnetic resonance (NMR) spectroscopies, and neutron scattering to fully study the glass-water interface.

ii. In addition, the use of $a b$ initio MD to calculate reaction paths and activation energy barriers for the hydrolysis of bridging oxygen bonds may improve upon the use of molecular clusters. This approach would allow the calculation of reaction paths that invoke the cooperative behavior of numerous water molecules in the dissolution process.

\subsubsection{Classical Molecular Dynamics (MD) Models}

Nangia et al. (2007) studied 40 unique hydroxylated $\beta$-cristobalite surfaces generated by cleaving the $\beta$-cristobalite unit cell along crystallographic planes. These surfaces are characterized by different ratios of $\mathrm{Q}^{1} \mathrm{Si}, \mathrm{Q}^{2} \mathrm{Si}$, and $\mathrm{Q}^{3} \mathrm{Si}$ as well as different surface topologies. Using classical MD simulations, H-bonding between the surface groups and between these groups and an 
overlying monolayer of water was investigated. Because of different surface topologies, two distinct $\mathrm{Q}^{3} \mathrm{Si}$ sites were defined. Stable $\mathrm{H}_{2} \mathrm{O}$ monolayers formed on some crystal surfaces but not on others. Argyris et al. (2008) investigated two slices through the (111) B-cristobalite surface with different hydroxyl surface density and therefore different degrees of hydrophobicity. Their results suggest that the first layer of water molecules at these surfaces are largely oriented hydrogen-down, and that the orientation of the second layer of water on the surface is dependent on the orientation of the first through hydrogen bonds. A second study by the same group (Argyris et al. 2009) studied one ß-cristobalite surfaces with three different degrees of hydroxylation. The structure of the first few layers of water overlying the silica surface differs depending on the degree of hydroxylation.

Du and de Leeuw (2006) used classical MD simulations to investigate hydration, dissolution, and nucleation processes at a quartz surface in water. From their investigation of quartz-water interactions, these researchers concluded that silica dissolution is an endothermic process, and that dissolved $\mathrm{Si}(\mathrm{OH})_{4}$ would remain near the surface and readily re-attach to the surface.

Numerous classical MD simulation studies have been performed to model glass structures and to examine the diffusion of ions in glass with time (Cabaret et al. 2001; Cormack et al. 2002; Du and Cormack 2004; Ganster et al. 2007; Pedone et al. 2006; 2008; Tilocca et al. 2006; 2007; Yuan and Cormack 2001). Some of these studies combine calculations with structure analyses. For example, Cabaret et al. (2001) studied medium range structure of borosilicate glasses with Si K-edge X-ray absorption near edge structure (XANES) spectra along with classical MD. Du and Cormack (2004) compared the calculated structure of sodium-silica glasses to neutron diffraction, extended X-ray absorption fine structure (EXAFS), and NMR data. Ferlat et al. (2006) examined the local environment of $\mathrm{Zr}$ in a borosilicate glass by combining MD simulations with Zr K-edge X-ray absorption spectroscopy (XAS) measurements. These types of comparisons are also used to improve the force field models for glass systems. Ganster et al. (2007) compared calculated structural and vibrational properties of a calcium aluminosilicate glass calculated with classical force fields and first-principles. Ispas et al. (2010) combined classical and Car-Parrinello MD simulations to investigate the structural properties of lithium tetrasilicate glass. The calculated structures were compared to experimental data concerning bond lengths and ${ }^{29} \mathrm{Si}$ MAS NMR spectra. The surface structure and hydroxylation of silica glass structures have also been studied using classical MD simulations (Du and Cormack 2005).

Glass surfaces will be less ordered than crystal surfaces and, to our knowledge, H-bonding between surface groups or between glass surfaces and overlying water layers has never been investigated in detail with classical MD. Cruz-Chu et al. (2006) developed a force field specifically for amorphous silica, but used contact angle information to evaluate the force field's capability to reproduce silica-water interactions. Car-Parrinello MD simulations and structural optimizations were used by Tilocca and Cormack (2008) to investigate the surface of a phosphosilicate glass and determine whether associative or dissociative adsorption of water occurs on the surface. Zeitler and Cormack (2006) investigated the interaction of water with bioactive glass surfaces. 


\subsubsection{Kinetic Monte Carlo (MC) Models for Dissolution}

Nangia and Garrison (2010b) have also started to use a kinetic MC approach to study the overall rate of dissolution and identify the intermediate species that have significant contribution of the dissolution process. In this approach, the master equation for the system is constructed from the rate equation of constituting reaction pathways. These equations result in a collection of coupled differential equations that are solved to obtain the time-propagation of the entire system. The solution of these equations gives the concentration of chemically active species as a function of time. One of the earliest stochastic kinetic MC approaches was developed by Gillespie (1976) for a two component system. This method can easily be extended to multicomponent systems.

The kinetic MC method assumes a priori knowledge of the reaction pathways and the corresponding rate constants. It also assumes that the initial concentrations of all the active chemical species are known. The first step of the kinetic MC procedure involves calculation of all the reaction rates from the rate constants and initial concentrations. The cumulative reaction rate is obtained by summing over rates of individual reactions obtained in the previous step. Then a reaction is selected using a random number such that the probability of selection is directly proportional to the rate of that reaction. The selected reaction is carried out by updating the concentrations of all the active species involved in the reaction according to their respective stoichiometry. The time step for this process is computed from the cumulative reaction rate and a random number between 0 and 1 using the following expression:

$$
\Delta t=-\frac{\ln u}{R}
$$

where $\mathrm{R}$ is the cumulative reaction rate, and $\mathrm{u}$ is a uniform random number between 0 and 1 . The rates of all the reaction are then updated and the procedure is repeated. As the simulation progresses, the concentration of various chemical species and the chemical rates can be monitored as a function of time. The scheme used by Nangia and Garrison (2010b) was developed by Dooling and Broadbelt (2001).

Nangia and Garrison (2010b) applied kinetic MC on a $10 \AA$ quartz crystallite embedded in a cubic box of water of $50 \AA$ dimension. The rate constant values for $\mathrm{k}_{\mathrm{p}}, \mathrm{k}_{\mathrm{n}}$, and $\mathrm{k}_{\mathrm{d}}$ were $6.6 \times 10^{-1}$, $6.5 \times 10^{-15}$ and $8.9 \times 10^{-5}$ calculated in Nangia and Garrison (2008) which is discussed in Section 3.1. The simulations were carried out in the $\mathrm{pH}$ range 4-8. The preliminary kinetic $\mathrm{MC}$ calculations on the crystallite result in a $\log$ (dissolution rate) that is within one order of magnitude with previously reported results. Nangia and Garrison plan to extend this method to include more topological information (i.e., $\mathrm{Q}^{1}, \mathrm{Q}^{2}$, and $\mathrm{Q}^{3}$ surface sites).

\subsubsection{Stochastic Monte Carlo (MC) Models for Dissolution}

MC methods are based on probabilities. The idea is that by repeating a stochastic process many times, one gets a clear average. MC methods can be applied to solve the mathematics of TST and study reaction kinetics. For example, consider the simple chemical reaction:

$$
\mathrm{A}+\mathrm{B} \rightarrow \mathrm{C}^{*} \rightarrow \mathrm{D}
$$


where the reactants $\mathrm{A}$ and $\mathrm{B}$ are forming the activated complex $\mathrm{C}^{*}$, which converts to the product $\mathrm{C}$. The kinetics of this equation is described by the probability $\mathrm{P}^{+}$to transform $\mathrm{A}+\mathrm{B}$ into $\mathrm{D}$ and the probability $\mathrm{P}^{-}$for the reverse reaction. At every time step, the $\mathrm{MC}$ method consists of transforming neighboring (A, B) to D particles with probability $\mathrm{P}^{+}$. Simultaneously, all $\mathrm{D}$ particles are transformed into $\mathrm{A}$ and $\mathrm{B}$ particles with the probability $\mathrm{P}^{-}$. This stochastic approach is a convenient approach to investigate the reaction kinetics of complex systems with several reactions and geometrical constraints (Aertsens and Ghaleb 2001).

\section{Quartz}

Most MC simulations used to investigate silica and aluminosilicate dissolution have focused on natural crystalline minerals. Nonetheless, the approaches used, the issues addressed, and many of the calculated results are directly applicable to amorphous silica and aluminosilicate glass dissolution. Nangia and Garrison (2009) developed a new MC algorithm to study the interplay between dissolution and precipitation reactions for quartz $\left(\mathrm{SiO}_{2}\right)$. The goal of the research was to understand how the local arrangement of reactive sites and surface topology impacted dissolution. Two possible mechanisms were tested for dissolution at neutral $\mathrm{pH}$ conditions. The mechanism that reproduced the experimentally-observed steady-state dissolution of the quartzwater system was defined as stepwise dissolution. The flow chart for stepwise dissolution is depicted in Figure 3-10. In this approach, only one $\mathrm{Si}-\mathrm{O}_{\mathrm{br}}-\mathrm{Si}$ bond is hydrolyzed at each $\mathrm{MC}$ step.

A protocol was developed to simulate a chemically realistic dissolution process. This protocol for bond hydrolysis requires knowledge of the $\mathrm{Q}^{\mathrm{i}}$ numbers of the selected Si site and its neighboring Si sites. In the case of $\mathrm{Q}^{2}$ and $\mathrm{Q}^{3}$ sites with more than one $\mathrm{Si}-\mathrm{O}_{\mathrm{br}}-\mathrm{Si}$ bond, it is more efficient to hydrolyze the bond with the least coordinated neighbor. For back reactions, the protocol is very general and picks precipitation sites stochastically, allowing both surface precipitation and polymerization in solution. Simulations invoking $\mathrm{Q}^{1}$-dissolution, $\mathrm{Q}^{1} \mathrm{Q}^{2}$ stepwise, and $\mathrm{Q}^{1} \mathrm{Q}^{2} \mathrm{Q}^{3}$-stepwise mechanisms all resulted in steady-state dissolution. They all yielded approximately the same $\mathrm{Q}^{0}\left(\mathrm{H}_{4} \mathrm{SiO}_{4}\right)$ fraction in solution and the $\mathrm{Q}^{4}$ bulk species fraction attained a nearly constant value. The $\mathrm{Q}^{2}$ and $\mathrm{Q}^{3}$ hydrolysis events were compensated by the backward precipitation reactions and did not contribute to the formation of $\mathrm{H}_{4} \mathrm{SiO}_{4}$. The amount of silicic acid in solution at steady-state depends on the number of initial $\mathrm{Q}^{1}$ sites. This result does not correspond to a thermodynamic solubility product. However, it may suggest that after the removal of $\mathrm{Q}^{1}$ sites, dissolution will be extremely slow. 
(after Nangia and Garrison 2010a)

\section{Figure 3-10. Flowchart for steps involved in the stepwise dissolution algorithm.}

The MC approach used combines reactive MC (Smith and Triska 1994; Johnson et al. 1994) for incorporating reactions and configurational bias MC (Siepmann and Frenkel 1992) to allow for the interchange of bulky surface groups with several water groups. To describe reactions where bonds are broken and formed, it is impossible to use an MC method that preserves the identity of the molecules in the system. Reactive MC conserves the number of atoms in a system and not the identity of individual molecules. According to Nangia and Garrison (2009), the forward dissolution for the silica-water system leads to the consumption of one water molecule per formation of an aqueous silicic acid molecule in bulk water, and the back precipitation reaction leads to the formation of water and the bridging of the free silicic acid back onto the mineral surface or polymerization in solution. The configurational bias MC technique provides an approach to carry out simulations of systems that involve components with very dissimilar densities and molecular structures. The Fueston-Garofalini potential energy function was used to simulate silicate-water interaction (Feuston and Garofalini 1990). This approach was used again by Nangia and Garrison (2010a) to investigate how the intrasurface hydrogen bonding on $B$ cristobalite surfaces impacts dissolution. The results suggest that hydrogen bonding of surface sites can change the preferential dissolution of $\mathrm{Q}^{1} \mathrm{Si}$ over $\mathrm{Q}^{2} \mathrm{Si}$ and $\mathrm{Q}^{3} \mathrm{Si}$. 


\section{Feldspar}

Lasaga and Luttge $(2003 ; 2004 a)$ introduced a general MC kinetic model for crystal dissolution that explicitly tracks all the various atoms in the crystal structure as part of the reaction mechanism. The model is based on a many-body reaction mechanism. It is built from both elementary reactions, i.e., bond-breaking and forming, and basic reactions, i.e., dissolution of surface units, adsorption and incorporation of solution units, and the mobility of units at the crystal surface. In Lasaga and Luttge (2004a), the theory is developed in detail for a crystal with a simple AB structure (e.g., halite), then, in Lasaga and Luttge (2004b), the theory is developed for the dissolution of an $\mathrm{A}_{3} \mathrm{~B}$ structure that is a cubic analogue for the more complex feldspar tetrahedral structure. In later papers, the theory is expanded to model the feldspar solid solution series.

The model uses a probabilistic approach to describe bond breaking and formation at a crystal surface. Lasaga and Luttge (2004a) define a basic reaction as the removal of a tetrahedral Si or Al group or unit from a feldspar structure. The basic reaction is a summation of their elementary reactions, and the probability of the basic reaction is a convolution of the probabilities of the elementary reactions that contribute to it. Lasaga and Luttge (2004a) start by supposing that the total number of A-A bonds on a reacting surface is $N_{A A}$, and that the total number of broken A-A bonds on the surface is $N_{A A}^{b r o k e n}$. Then at steady state,

$$
\frac{N_{A A}^{\text {broken }}}{N_{A A}}=\frac{k_{-}}{k_{+}}
$$

where $k_{-}$and $k_{+}$are the rate constants for the breaking and formation of an A-A bond. If only a few bonds are broken at any one time, then $N_{A A} \sim N_{A A}^{t o t} \sim$ constant, and the probability that any given $\mathrm{A}-\mathrm{A}$ bond is broken is given by

$$
P_{A A}=\frac{k_{-}}{k_{+}}
$$

The rate of dissolution of units with $n$ bonds will depend on the number of A-units with $n$ bonds on the surface $N_{n}^{A}$. It will also depend on the probability that all $n$ bonds are found broken at the same time:

$$
\text { Dissolution Rate }=\left(P_{A A}\right)^{n} N_{n}^{A} \text {, or Rate }=\left(\frac{k_{-}}{k_{+}}\right)^{n} N_{n}^{A}
$$

The individual rate constants, for breaking or forming an A-A bond are elementary rate constants; therefore, it can be shown that the ratio of the rate constants obeys the TST relation,

$$
\left(\frac{k_{-}}{k_{+}}\right)=e^{\Delta E / k T}
$$

where $\Delta E$ is the energy difference between the bond and broken-bond states, $k$ is the Boltzmann's constant, and $T$ is temperature. A significant difference between the $\mathrm{MC}$ approach and the TST approach describing dissolution is that the activation energy for the overall basic dissolution reaction of a unit with $n$ bonds, $E_{a c t}^{n}$ does not reflect the activation energies needed to 
break individual bonds, but the number of bonds, $n$, and the energy difference between an original bond and a broken bond

$$
E_{a c t}^{n}=n \Delta E
$$

This is also true of the stochastic MC approach used by Nangia and Garrison (2008) for quartz. The activation energy barriers for individual bond-breaking and making are not considered in the overall basic dissolution model. Because all rates or probabilities in a MC treatment need to be tied to some time scale, a general frequency factor, $v$, is introduced for bond-breaking. The change in energy, $\Delta E$ for one bond is labeled $\Phi$ and the dissolution rate of blocks with $n$ bonds is given by

$$
k_{n}^{-}=v e^{-n \Phi / k T}
$$

The frequency factor $v$ is determined from experimental data and ultimately the exact meaning of $\Phi$ and its size need to be determined through $a b$ initio calculations.

As an example, for a surface site with 3 bonds at the $\mathrm{AB}$ surface, the rate of arrival $k_{+}$at equilibrium is given by:

$$
k_{e q}^{+}=k_{3}^{-}=v e^{-3 \Phi / k T}
$$

If the concentrations of species in solution are not equilibrium concentrations, then

$$
\frac{c_{A}}{c_{A, e q}}=e^{\Delta \mu_{A} / k T} \text { and } \frac{c_{B}}{c_{B, e q}}=e^{\Delta \mu_{B} / k T}
$$

The rate of arrival of A- and/or B-atoms at the surface depends on the concentration of A- and Batoms in solution at the surface. Then, the final expression is:

$$
\begin{aligned}
& k_{A}^{+}=v e^{-3 \Phi / k T} e^{\Delta \mu_{A} / k T} \\
& k_{B}^{+}=v e^{-3 \Phi / k T} e^{\Delta \mu_{B} / k T}
\end{aligned}
$$

In this example, both A and B have the same type of surface site and the same related energetics $(3 \Phi)$. The arrival rates of A- and B-units to the surface are not coupled in this model. The rate at which either unit $\mathrm{A}$ or $\mathrm{B}$ arrives at the surface is dictated by the concentration of $\mathrm{A}$ or $\mathrm{B}$ in solution $\left(\Delta \mu_{\mathrm{A}}\right.$ and $\left.\Delta \mu_{\mathrm{B}}\right)$.

Lasaga and Luttge (2004a) also introduce a rate constant to describe the diffusion of atoms (i.e., $\mathrm{Q}^{1}$ surface species) along a crystal surface during dissolution:

$$
k_{a d}=v e^{-E_{a d} / k T}
$$


The pre-exponential frequency factor for surface diffusion is assumed to be the same as for dissolution. The surface diffusion rate for a surface species with $\mathrm{n}_{\text {lat }}$ lateral bonds in the simple AB-case is:

$$
k_{\text {diff }}=v e^{-E_{a d} / k T} e^{-n_{\text {lat }} \Phi / k T}
$$

In summary, Lasaga and Luttge (2004a) demonstrate that the overall dissolution rate can be defined as a statistical average of basic processes over time and over an entire surface. The "basic reactions" in the statistical dissolution process of Lasaga and Luttge (2004a) consist of the arrival or departure of individual molecular units. The activation energy for crystal dissolution reflects the energy difference, $\Delta E$, between hydrated bonds and hydrolyzed broken bonds - not the activation energies for individual bond-making and breaking elementary reactions.

One outcome of this model is that the individual attachment and detachment processes become coupled and give rise to the solubility product as the main control over the dissolution rate near equilibrium. This suggests that the model can describe dissolution rates both near and far from equilibrium. Lasaga and Luttge (2005) investigate how the solubility product arises from the coupling that occurs between the concentration in solution and the distribution of surface sites, using albite as an example:

$$
\begin{array}{r}
\mathrm{NaAlSi}_{3} \mathrm{O}_{8}+8 \mathrm{H}_{2} \mathrm{O} \leftrightarrow \mathrm{Na}^{+}+\mathrm{Al}(\mathrm{OH})_{4}^{-}+3 \mathrm{H}_{4} \mathrm{SiO}_{4} \\
K_{S p}=a_{\mathrm{Na}^{+}} a_{\mathrm{Al}(\mathrm{OH})_{4}^{-}} a_{\mathrm{H}_{4} \mathrm{SiO}_{4}}^{3}
\end{array}
$$

To illustrate the kinetic approach, Lasaga and Luttge (2005) use a crystal-based reaction mechanism to justify the growth or dissolution of a simple feldspar-like layer and derive the solubility product from kinetics:

$$
\frac{d N_{A l}^{k}}{d t}=k_{A l}^{+} N_{S i_{1}}^{k}+k_{S i_{3}}^{-} N_{S i_{3}}^{k}-k_{A l}^{-} N_{A l}^{k}-k_{S i}^{+} N_{A l}^{k}
$$

The first term is the rate of arrival of $\mathrm{Al}$ atoms at $\mathrm{Si}_{1}$ kink sites, thereby producing an $\mathrm{Al}$ kink. $\mathrm{Si}_{1}$ kink sites are the only sites where Al atoms can attach to the structure (see Figure 3-11). The parameter $k_{A l}^{\mp}$ is the attachment rate constant for $\mathrm{Al}$ atoms from solution which depends on $\mathrm{C}_{\mathrm{Al}}$.

Similar equations are used for $N_{\text {Si1 }}^{k}, N_{\text {Si2 }}^{k}, N_{\text {Si3 }}^{k}$, where Al atoms can attach to $\mathrm{Si}_{1}$ sites, $\mathrm{Si}_{1}$ are attached to $\mathrm{Si}_{2}$ sites that neighbor $\mathrm{Si}_{1}$ and $\mathrm{Si}_{3}$ sites, and $\mathrm{Si}_{3}$ sites are next to Al kink sites:

$$
\frac{d N_{A l}^{k}}{d t}+\frac{d N_{S i_{1}}^{k}}{d t}+\frac{d N_{S i_{2}}^{k}}{d t}+\frac{d N_{S i_{3}}^{k}}{d t}=0
$$




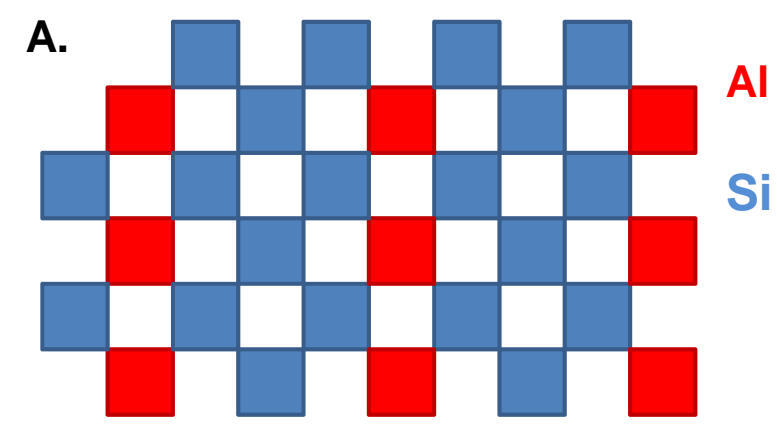

fluid

\section{B.}

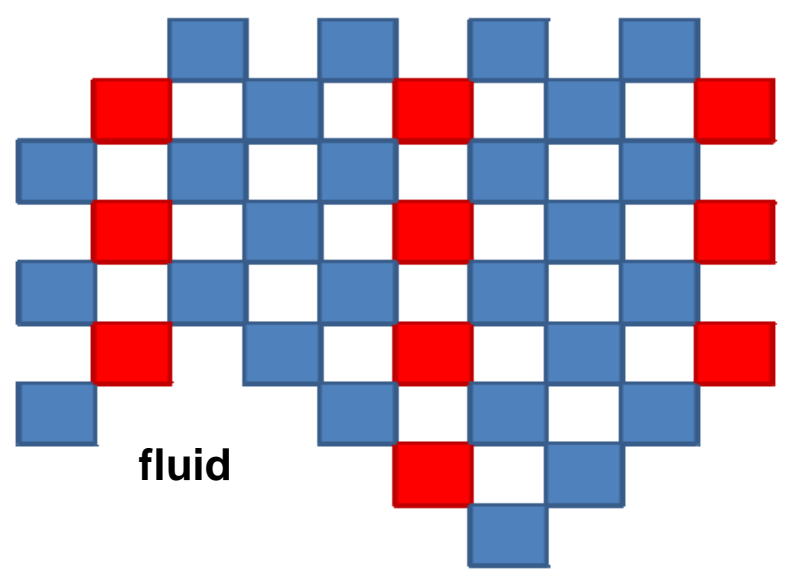

C.

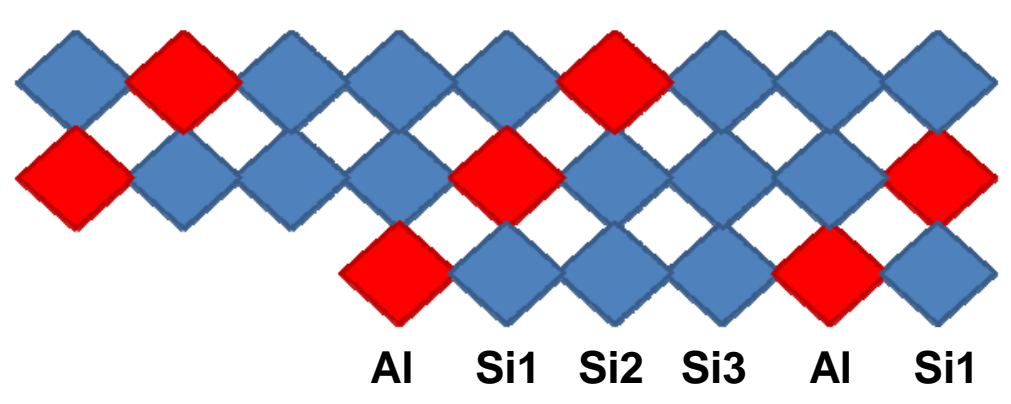

(A) The four coordinated framework is displayed in two dimensions. The $\mathrm{AlSi}_{3}$ stoichiometry is representative of the feldspar series. The reactive surface shown consists of all $\mathrm{Q}^{2}$ sites requiring two new bonds to attach a new unit.

(B) Surface in (A) after reaction with a fluid. The reactive sites grow (or dissolve) quickly to produce new surfaces.

(C) A diagonal face of the model crystal which grows or dissolves in an orderly step-controlled fashion. An Al kink site is shown. Note the different kink sites produced by the removal of the Al kink atom and subsequent $\mathrm{Si}$ atoms along the step. These kink sites are labeled $\mathrm{Al}, \mathrm{Si}_{1}, \mathrm{Si}_{2}$, and $\mathrm{Si}_{3}$.

(after Lasaga and Luttge 2005)

Figure 3-11. Simplified model of a dissolving feldspar surface. 
The overall dissolution rate $(O D R)$ is obtained from the net difference between attachment and detachment of the various atoms to the surface kink sites:

$$
O D R=k_{A l}^{-} N_{A l}^{k}+k_{S i_{1}}^{-} N_{S i_{1}}^{k}+k_{S i_{2}}^{-} N_{S i_{2}}^{k}+k_{S i_{3}}^{-} N_{S i_{3}}^{k}-k_{A l}^{+} N_{S i_{1}}^{k}-k_{S i}^{+}\left(N_{A l}^{k}+N_{S i_{2}}^{k}+N_{S i_{3}}^{k}\right)
$$

The energy change needed to rupture a $\mathrm{Si}-\mathrm{O}-\mathrm{Al}$ bond is $\varphi_{\mathrm{SiAl}}$. Because an $\mathrm{Al}$ atom in an $\mathrm{Al}$ kink site has two $\mathrm{Si}-\mathrm{O}-\mathrm{Al}$ bonds, the detachment rate $k_{A l}^{-}$is given by:

$$
k_{A l}^{-}=v_{1} e^{-2 \varphi_{\mathrm{SiAl}} / \mathrm{kT}}
$$

where $v_{1}$ is a frequency parameter, related to the time scale, and in general obtainable from experiment.

The $\mathrm{Al}$ attachment rate depends on the average kink site in the crystal structure and the solution concentration. Therefore,

$$
k_{A l}^{+}=v_{1} e^{-2 \varphi_{\mathrm{SiAl}} / \mathrm{kT}} e^{\Delta \mu_{A l} / k T}
$$

Continuing with this analysis, the final expression for the overall rate becomes:

$$
\text { Rate }=N_{\text {tot }}^{k} \frac{4}{S} k_{A l}^{-} k_{S i_{1}}^{-} k_{S i_{2}}^{-} k_{S i_{3}}^{-}\left(1-e^{\Delta G / R T}\right)
$$

where $N_{\text {tot }}^{k}$ is the total number of kinks on the surface, $\mathrm{S}$ is a function of all the attachment and detachment rate constants, and $\Delta G=3 \Delta \mu_{S i}+\Delta \mu_{A l}$.

The significance of the $\Delta G$ term in Equation 3.3.26 is that it shows that $\Delta G$ controls the rate as equilibrium is approached, such that the Rate $\rightarrow 0$ as $\Delta G \rightarrow 0$. Using this expression, Lasaga and Luttge (2005) successfully analyzed the data for Al inhibition of the K-feldspar dissolution rate of Gautier et al. (1994) and also provided a fundamental justification for product-like phenomenological rate laws.

Continuing with this model, Zhang and Luttge (2007) examined the role of (Al, Si) disorder on the kinetics of albite dissolution. Two different dissolution mechanisms - multilayer leaching and single-layer retreat - were tested on 48 albite configurations. In simulations involving multilayer leaching, dissolution rates increased with decreasing long-range order. A fivefold increase in dissolution rate tied to $(\mathrm{Al}, \mathrm{Si})$ disordering was simulated. This increase is comparable to that accompanying a decrease of $1.4 \mathrm{pH}$ units at $\mathrm{pH}$ 1-5. This rate increase due to disordering in albite is also similar to that observed from compositional variation in plagioclase feldspars $\left(\mathrm{An}_{47} \mathrm{vs}\right.$. $\left.\mathrm{An}_{0}\right)$ at low $\mathrm{pH}$. In contrast, (Al, Si) disordering had no discernible effect during the simulations involving the single-layer retreat mechanism. These results suggest that the effect of (Al, Si) disorder on albite dissolution rate is mechanism dependent. Zhang and Luttge (2008) used stochastic modeling to compare experimental results regarding the saturation state dependence of the dissolution rate, aluminum inhibition, and surface chemistry evolution. The model shows that inhibition of $\mathrm{Si}$ atoms in solution governs the kinetic effects relatively close to equilibrium, while the inhibition of $\mathrm{Al}$ atoms in solution controls the kinetic effects at 
far-from-equilibrium conditions. The stochastic approach also suggests the appearance of an alteration silica-rich gel layer on the aluminosilicate surface as dissolution progresses.

In Zhang and Luttge (2009), the more rapid dissolution of Ca-rich feldspars relative to Na, Krich feldspars is examined. This more rapid dissolution has been attributed to the preferential leaching of $\mathrm{Al}$ deep within the feldspar structure by some investigators. However, evidence from surface microanalysis (e.g., Hellmann et al. 2003), shows that preferential dissolution of Al is confined to the top layers of the feldspar lattice and that the amorphous surface layer most likely results from precipitation versus dissolution. Using stochastic MC simulations, two different dissolution mechanisms are tested using compositions representing the entire plagioclase solid solution series. The first dissolution mechanism involves preferential leaching of Al. The modeling shows that the influence of ( $\mathrm{Al}, \mathrm{Si}$ ) disorder on the dissolution rate is significant. At a fixed composition, an increase in ( $\mathrm{Al}, \mathrm{Si})$ disorder results in an increased dissolution rate that is composition dependent. In the second set of simulations, Al removal is confined to the top surface layers. A variety of feldspar configurations with different (A1, Si) disorder but a single fixed composition are calculated to have similar dissolution rates. The dissolution rate of $\mathrm{Ca}-$ rich (anorthite-rich) feldspars departs positively from its log-linear relationship around $\mathrm{An}_{80}$ which is in good agreement with experimental studies. Subsequent modeling results of Al inhibition, $\Delta G$ dependence, and formation of altered surface layers are all comparable with experimental investigations, suggesting that an interfacial dissolution-reprecipitation mechanism governs the dissolution of plagioclase feldspars.

\section{Generic and Nuclear Waste Glass}

MC simulations were also done by Bandstra and Brantley (2008). These researchers focused on examining the possible effects of surface evolution by simulating surface area and dissolution rate as a function of time and $\Delta \mathrm{G}_{\mathrm{rxn}}$ for a simple model - a binary random Markov field - where complexities normally associated with detailed reaction mechanisms have been removed so that the resulting behavior is entirely caused by surface evolution. The model consists of a simple periodic crystal structure that constrains the topography of the surface. Dissolution reactions are stochastic processes with probabilities uniquely determined by the local compositional environment of a given surface site. Information on the local arrangement of atoms on the surface is used to determine the number of bridging neighbors and based on this number the dissolution and precipitation probabilities are calculated. The dissolution process is reversible via precipitation reactions whose probabilities are determined by both the local compositional environment of the reaction site and the saturation state of the solution (i.e., free-energy of reaction $\Delta \mathrm{G}_{\mathrm{rxn}}$ ). The minerals contain one- and two-dimensional defects (i.e., dislocations and grain boundaries) where dissolution and precipitation reactions are more likely. Based on these features, the two-dimensional system shows that mineral surfaces reach a steady-state conformation with time. Several surface conformations were studied and it was found that each surface evolved to an equilibrium conformation as a function of time. Initial surface roughness disappeared as steady-state was achieved.

MC simulations have also been considered specifically for nuclear waste glass dissolution. Aertsens (1999) specifically applied a MC method to investigate the dissolution kinetics of a simplified glass and compare the results to the linear Grambow rate law (1987). He linked the macroscopic parameters in the Grambow rate law to the microscopic parameters in the $\mathrm{MC}$ 
dissolution model and concluded that a linear, congruent model of glass dissolution was insufficient.

\subsubsection{Differences, Gaps, and Uncertainties}

The MC techniques discussed above have not yet been implemented for glass dissolution studies, but could provide a very useful to way to incorporate a database of activation energies for bond breaking between different glass components and introduce actinides and radionuclides into the glass structure. The use of stochastic and kinetic MC approaches to study aluminosilicate dissolution is relatively new. The stochastic MC approach has been used successfully to model the dissolution of several different feldspar minerals. In each study, several different scenarios are tested and compared to experimental results. It would be useful to evaluate these scenarios for aluminosilicate glasses of different compositions, and extend this approach to multicomponent nuclear waste glasses. At this point it appears that Nangia and Garrison (2010a) are the first to start developing a kinetic MC approach to study silica dissolution processes. These MC approaches look very promising for bridging the atomistic and continuum scales, particularly because they can be used to reproduce both near- and far-from equilibrium dissolution and the start of gel layer formation on an aluminosilicate surface. One area of research to consider is how this approach can be used to more fully describe gel layer formation and whether it can be extended to include calculations of gel layer porosity. It should be pointed out that the MC methods are used to test different conceptual models for dissolution by comparison against experimental data. Therefore, the predictive capabilities of the approach strongly depend on the validity of the embedded conceptual model. Nonetheless, these approaches look very promising for investigating multicomponent nuclear waste form dissolution processes.

\subsubsection{Modeling Mesoscale Effects on Glass}

A number of additional issues related to the glass physical configuration and chemical composition provide further potential gaps to be addressed. Researchers have been studying the dissolution of aluminosilicate minerals and glass for decades now, but have not studied as extensively the impact of mixing nuclear waste into the glass on nuclear waste form lifetime and corrosion processes. Grambow (2006) points out that commercial high-level waste immobilized in the glass is composed of over 30 different nuclear fission and activation products, as well as minor actinides generated by neutron capture reactions. A number of these radionuclides have long half-lives, creating the need for long-term isolation. Radionuclides like ${ }^{137} \mathrm{Cs} /{ }^{137 \mathrm{~m}} \mathrm{Ba}$ and ${ }^{241} \mathrm{Am}$ remain important heat sources for hundreds to thousands of years. The fracturing of glass blocks during cooling after pouring leads to additional fractures and surface area increases of as much as 50 times greater than the external surface area. Volcanic glasses persist in natural environments, with little evidence of devitrification to crystalline phases and nuclear waste glasses are also unlikely to devitrify in the temperature range $\left(<450^{\circ} \mathrm{C}\right)$ envisioned for interim storage, transport and disposal, but may undergo processes that generate fresh fractures and additional surface area.

Verney-Carron et al. (2010) coupled glass alteration models with diffusive transport in solution to simulate alteration in cracks within a fractured block of Roman glass that has been altered for 
1800 years on the sea floor. External cracks in direct contact with seawater were more altered than internal cracks (by 1 to 2 orders of magnitude). The alteration of the internal cracks shut off because of the strong coupling (negative feedback) between alteration chemistry and transport in the cracks. The archeological glass has a cracking factor of $86 \pm 27$ (i.e. the surface area developed by the cracks is 86 times greater than the geometric surface) and a total of $12.2 \pm$ $4.1 \%$ of the glass is altered (Verney-Carron et al. 2010). However, only the external cracks alter at what may approximate a constant rate. The internal crack network (responsible for the major fraction of the reactive surface area) is altered at a rate that decreases over time. Over longer time periods the discrepancy between the alteration kinetics of the external and internal cracks should increase and the contribution of the internal cracks to the overall alteration should decrease accordingly. At present nuclear glass models used for performance assessment assume that the amount of altered glass will remain proportional to the initial surface of the glass blocks (including internal cracks) until complete alteration is reached (e.g., Gin et al. 2005). Based on Verney-Carron et al. (2010) this assumption probably overestimates the true extent of alteration.

Another issue is the possibility of rapid diffusion and loss of radionuclides from the glass even under dry conditions (Grambow 2006). Solid-state tracer diffusion coefficients of $\mathrm{Sr}$ and $\mathrm{Cs}$ in borosilicate glass extrapolated to the upper limit of disposal temperatures $\left(<100^{\circ} \mathrm{C}\right.$ in many repository systems) are too slow for any significant mobility even over millions of years (Grambow 2006). If suitable cast-iron overpacks (ANDRA 2005) are used, glass-groundwater contact during the time interval over which the waste form remains hot will not occur.

While Grambow (2006) discusses current repository and waste form concepts, Weber et al. (2009) develops ideas for the wastes that would be generated by recycled nuclear fuel. Recycling nuclear fuel would greatly reduce the volume and activity of nuclear waste needing geologic disposal prior to complete decommissioning. Waste streams that might require separate waste forms include (1) the fission product ${ }^{99} \mathrm{Tc}$ (half-life of $2.1 \times 10^{6}$ years), (2) heat-generating isotopes ${ }^{137} \mathrm{Cs}$ and ${ }^{90} \mathrm{Sr}$ (half-lives of 30.2 and 28.8 years, respectively); (3) lanthanides; (4) minor actinides (half-lives from 10 to $10^{6}$ years); (5) the remaining fission products (half lives from 1 to $10^{7}$ years); (6) the volatile radionuclides ${ }^{3} \mathrm{H},{ }^{129} \mathrm{I},{ }^{14} \mathrm{C}, \mathrm{Xe}$ and $\mathrm{Kr}$ (half-lives from 10 to $10^{7}$ years); and (7) the undissolved solids from fuel dissolution.

Self radiation from radionuclide decay can affect the microstructural evolution, phase stability, and thermodynamic properties in waste forms. The principal sources of radiation in HLW are $\beta$ decay of the fission products (e.g., ${ }^{137} \mathrm{Cs}$ and ${ }^{90} \mathrm{Sr}$ ) and a decay of the actinide elements (e.g., U, $\mathrm{Np}, \mathrm{Pu}, \mathrm{Am}$, and $\mathrm{Cm})$. Beta-decay produces energetic $\beta$-particles $(\sim 0.5 \mathrm{MeV})$, low-energy recoil nuclei, and $\gamma$-rays; whereas $\alpha$-decay produces energetic $\alpha$-particles (4.5-5.5 MeV), energetic recoil nuclei $(70-100 \mathrm{keV})$, and some $\gamma$-rays. These particles and $\gamma$-rays interact with solids primarily through energy transfers to electrons through ionization processes, producing electronhole pairs, or to atomic nuclei through elastic collisions, displacing atoms to produce defects. In general, $\beta$-decay of the short-lived fission products is the primary source of radiation (and heat generation) from HLW during the first 600 years of storage. Because of the long half-lives of the actinides and their daughter products, $\alpha$-decay is dominant at longer times.

Based on years of radiation-effects research on nuclear waste forms, only radiation effects from actinides and the fission products $\mathrm{Cs}$ and $\mathrm{Sr}$ are expected to be of major concern. In actinide- 
bearing waste forms, helium atoms formed from $\alpha$-particles must be accommodated. For high actinide loadings, the $\mathrm{He}$ concentrations can become quite high $(>1 \%)$ and difficult to accommodate within both glass and ceramic structures. In glass waste forms, at the ambient temperature expected over the decay times for actinides, the effects of $\alpha$-decay are generally small. There are small changes in stored energy, structure and volume with decay, but for the most part glasses generally do not exhibit significant changes in chemical durability.

The proposed use of separate $\mathrm{Cs} / \mathrm{Sr}$ waste forms would allow for interim storage over several hundred years. Because of desired high waste loading, $\mathrm{Cs} / \mathrm{Sr}$ waste forms will be subject to significant self-heating (up to $1000^{\circ} \mathrm{C}$ ) and high ionization and transmutation rates. The cumulative ionization dose projected for $\mathrm{Cs} / \mathrm{Sr}$ waste forms is higher than in commercial HLW glass. At these doses and temperatures, many materials undergo decomposition, phase separation and bubble formation. Developing predictive models and validating such an approach will require a fundamental understanding of ionization effects and the coupling of electronic excitations to atomic dynamics. However, the intent is to allow for waste form cooling during storage prior to disposal in a nuclear waste repository (Weber et al. 2009).

Radiation effects on specific surface characteristics and on dissolution processes have not been well studied at the molecular level. However, it is anticipated that these effects will accelerate dissolution by breaking local bonds, increasing surface areas, and modifying reaction paths. This is a topic that requires more investigation.

\subsubsection{Experimental Validation of Molecular Models}

Several methods may be used to help determine reaction mechanisms. Experiments determining rate laws, activation energies, or isotope exchange kinetics provide insight into steps along the reaction path. Spectroscopic studies can elucidate the structure of intermediate species, which provide clues to the reaction mechanism (Kubicki 2008).

Many reaction rates are conditional on the experimental setup. Extrapolation to the field is challenging because degradation rates observed in the field tend to be much slower than in the laboratory. By identifying the rate-determining species and the activated, or transition state, complex, one can determine which factors may play a key role in constraining the reaction rate in the field. The difference in free energy between the reactants and a transition state complex is the key controlling factor in the overall rate of reaction, for simple elementary reactions. The recent development of femtosecond laser spectroscopy has allowed identification of species that can be realistically called transition state complexes (e.g., Gilijamse et al. 2005; Abramczyk et al. 2006). Application of these techniques might be useful to examine key reactions in nuclear waste glass dissolution.

\subsubsection{Methods for Determining Mechanisms}

Ideally, a glass dissolution rate law would reflect the elementary rate-controlling step of the reaction and the mechanism would be fairly clear from this determination alone. However, in complex geochemical reactions, comprised of numerous elementary steps, the reactant and product concentrations may be a function of unknown and unmeasured species not represented in 
the rate law. Species not included in the overall reaction may control the reaction rate. Experiments that measure rates over a range of temperature can be used to calculate overall activation energies. These activation energies provide clues as to the rate-determining step of the reaction. For example, the activation energies for breaking covalent bonds are on the order of $100 \mathrm{~s} \mathrm{~kJ} / \mathrm{mol}$ while diffusion of $\mathrm{H}_{2} \mathrm{O}$ through minerals requires $10 \mathrm{~s} \mathrm{~kJ} / \mathrm{mol}$. A given activation energy does not indicate a unique bond breaking mechanism (Kubicki 2008).

Isotopic tracers can identify atom types in a reaction. Labeling certain reactants with enriched isotopic values can be valuable in recognizing important steps of the reaction mechanism. NMR kinetic studies using isotopically labeled species can be used to follow steps in aqueous exchange reactions (e.g., Yu et al. 2003; Lee and Stebbens 2003).

Complex reactions consisting of numerous steps may result in the formation of reactive or metastable intermediate species. These species may be amenable to identification via spectroscopic techniques and can help constrain important steps with the reaction pathway. For example, mineral and glass surfaces resulting from dissolution experiments (Hamilton et al. 2001) were analyzed using ${ }^{1} \mathrm{H}^{27} \mathrm{Al}$ Cross-Polarization Magic-Angle Spinning Nuclear Magnetic Resonance (CP-MAS-NMR) spectroscopy (Tsomaia et al. 2003). This technique enhances the signal of surface $\mathrm{Al}$ atoms over those in the bulk because only $\mathrm{Al}$ atoms with connections to $\mathrm{H}$ atoms are observed. NMR spectra of bulk feldspar samples detected only tetrahedral Al, but an NMR peak due to octahedral Al species connected to $\mathrm{OH}$ or $\mathrm{OH}_{2}$ groups on the surface of the glass was observed in CP- MAS-NMR spectra. Subsequently, Criscenti et al. (2005) suggested that formation of octahedral Al could be part of the dissolution mechanism because hydrolysis of ${ }^{[6]} \mathrm{Al}-\mathrm{O}-\mathrm{Si}$ linkages should require less energy than ${ }^{[4]} \mathrm{Al}-\mathrm{O}-\mathrm{Si}$ linkages based on bond length/bond strength arguments. Calculated ${ }^{27} \mathrm{Al}$ NMR chemical shifts of ${ }^{[6]} \mathrm{Al}$ linked to three silica tetrahedral were in agreement with the measured values indicating that the coordination change could occur in situ on the mineral surface. Recent transmission electron microscopy work of Hellmann et al. (2003) has been interpreted to suggest that this tetrahedral to octahedral coordination change may occur in the near-surface solution and then reprecipitate on the surface.

\subsubsection{Measuring Changes in Surface Topography, Chemistry, and Structure}

An overview of experimental techniques that can be used to quantify surface topography is provided by Luttge and Arvidson (2008). One method used to measure the dynamics of surface dissolution is atomic force microscopy. AFM is a high-resolution imaging technique that can be used to track the motion of monomolecular steps in situ, as a function of solution composition in a flow-through environment. It is the collective motion of steps on the surface that accounts for the macroscopic behavior observed in a given solution and it is at this scale that gaps in our understanding of the bulk system may be most directly addressed. The small length-scale of observation has the added advantage that changes in solution chemistry are immediately detectable at the surface, reducing measurement acquisition times and maximizing the precision by which solution chemistry can be controlled.

Interferometry is another surface topography technique that can provide data over a wide range of length scales. Mirau or Michaelson interferometric techniques can be used to quantify surface topographies at the $\mathrm{nm}-\mathrm{cm}$ scale. Luttge et al. (1999; 2003) and Luttge (2004) introduced 
vertical scanning interferometry and its application to mineral dissolution and growth. High vertical resolution at the $\AA-\mathrm{nm}$ scale is achieved by adding a piezoceramic to the interferometer for a z-scan of the sample. The lateral resolution depends on the Mirau objective used. The field of view is generally larger than for an AFM; a surface area of almost $1 \mathrm{~mm}^{2}$ and up to $20 \mu \mathrm{m}$ of surface relief can be quantified in several seconds. The measurements of surface-normal height changes can be converted directly into dissolution or growth rates and averaged over the entire solid surface. This leads to a rate constant that is independent of any external measurement of the surface area. One scan provides typically up to 100,000 height data points. These types of data lead directly to conceptualizing surface dissolution as a statistical process and developing a stochastic MC approach (e.g., Luttge 2004) for describing this process. Icenhower, et al. (2003) used this experimental approach to study the dissolution of borosilicate glasses designed for nuclear waste stream disposal.

A third technique that has been used to study surface dissolution is scanning electron microscopy. Typically, SEM produces images of a sample surface that resolve surface features and microtopography. Environmental SEM allows for in situ exploration of the surface (i.e., in water), providing new opportunities to study dissolution processes.

Surface chemistry and structure are analyzed using XPS, SIMS, and X-ray (grazing incidence) diffraction. XPS consists of measuring the kinetic (binding) energy of photoelectrons produced during interaction with an X-ray source. Electrons that escape the surface for detection originate from the uppermost few monolayers. XPS is sensitive to the elemental composition as well as the chemical environment of these elements. SIMS is a highly sensitive surface technique for characterizing materials, and can be used a complementary technique to XPS. The principle of the method is based on the mass analysis of secondary ions created through the interaction of a beam with the surface of a solid. Grazing incidence X-ray diffraction can be used to study surface layers if the incident beam is confined to a very shallow angle with the surface (Luttge and Arvidson 2008).

\subsubsection{Summary of Gaps in Upscaling Dissolution Processes}

Recent modeling research to understand aluminosilicate dissolution mechanisms has focused on two areas: (1) using $a b$ initio cluster calculations to quantify the activation energy barriers for proposed reaction paths for dissolution processes, and (2) developing and implementing stochastic Monte Carlo methods that can be used to simulate different dissolution mechanisms for comparison to experimental data. To calculate activation energies for elementary reactions there are several $a b$ initio approaches in use that reflect differences in surface and reaction path conceptualization. For example, should the bonds at the surface be considered fully flexible or should they be constrained according to the material surface and should explicit water molecules be included in the molecular cluster and consequent reaction path? It has also been demonstrated that the stochastic MC methods can account for: a) dissolution both near- and far-from equilibrium; b) observed changes in dissolution over time from rapid dissolution to steady-state dissolution; c) dissolution of aluminosilicate phases of various compositions and with different $\mathrm{Al}$ and Si ordering; and d) potentially, the development of gel layers. However, these MC methods rely heavily on conceptual roadmaps of the dissolution process, and not directly on 
atomistic data. More computational research as well as experimental validation is necessary to link these two modeling approaches.

Simulating the degradation of glass nuclear waste forms is even more challenging in part because of the larger chemical system of glass. The glass matrix contains additional components such as boron and zirconium. The nuclear waste adds additional compositional variability to the system including radionuclides like ${ }^{137} \mathrm{Cs} /{ }^{137 m} \mathrm{Ba}$ and ${ }^{241} \mathrm{Am}$ that can remain important heat sources for hundreds to thousands of years. Radiation effects on dissolution processes have not been investigated extensively at the molecular level, but will most likely accelerate the breaking of local bonds, increase reactive surface area by encouraging crack formation, and possibly alter reaction mechanisms. Although the impact of cracks internal to glass structure have been studied by Verney-Carron et al. (2010) for one scenario, other scenarios should also be investigated to develop additional evidence of the potential effect of crack development on glass waste form degradation.

\subsection{Verification, Validation, and Uncertainty Quantification}

Verification of data acquisition and simulation codes, validation of simulation results, and quantitative assessment of uncertainties must become an integral part of all sub-continuum-scale activities that support development of constitutive models for use in continuum simulations. These considerations are reflected in the discussions above concerning gaps in scientific capabilities. Adequate V\&V and UQ practices are not yet well-established standard practice for experiment and simulation methods at the sub-continuum scale. The needs are to institute sufficiently rigorous $V \& V$ standard and practices for all data emerging from simulation studies at the sub-continuum scale, and develop methods for upscaling those results into higher scales of fidelity, with quantitative propagation of uncertainties through the hierarchy of phenomena scales. The workflow and data that is used to generate higher-scale models need to be collected, assessed, and recorded.

\subsubsection{Practices for Sub-Continuum-Scale Modeling}

All data used and generated by sub-continuum scale activities - verification and validation evidence in support of simulations, computational model constructions, etc. - will need to be properly qualified: data and codes verified, simulation predictions validated against data, and uncertainties quantitatively assessed.

The phenomena of interest range from electronic chemical properties - requiring quantum chemical codes, to dissolution and transport - entailing (near-continuum) reactive transport codes. There is no single code that spans the full range of sub-continuum modeling needs, indeed, there are multiple codes within each class of phenomena. Codes commonly used within this scale can be commercial, open source, or private codes that are not distributed. Furthermore, additional modeling tools might need to be developed to fill gaps in scientific capabilities, e.g., to enable upscaling. The Waste IPSC will generally not develop codes specific to subcontinuum modeling, any methods development efforts will be coordinated with the FMM program element. 
A full inventory of all possible codes at the sub-continuum scale that might ultimately feed the workflow of a waste disposal system assessment is not feasible. The Waste IPSC cannot and will not anoint codes at the sub-continuum scale, but operation of the Waste IPSC can and will impose standards on the generation of data from sub-continuum scales that will be used in any workflow analysis. Existing published results often lack verification evidence or validation data, and have minimal or no discussion of uncertainties. Moreover, the importance of different subcontinuum phenomena to the PA-scale model is not yet well developed, and the level of formality that may be demanded of particular simulations is undetermined. For sub-continuum processes to contribute meaningfully to construction of continuum-scale models, protocols for $\mathrm{V} \& \mathrm{~V}$ and UQ need to be defined and demonstrated to replace the ad hoc practices that currently exist.

An obvious early need for the sub-continuum scale is to establish clear expectations and guidelines for verification and validation practices across sub-continuum scale with appropriate consideration of uncertainties, calibrated to the importance of the phenomena being modeled. This gap will be addressed in a FY11 Milestone that will discuss V\&V and UQ guidelines for sub-continuum scale modeling. This will involve collating a gross inventory of classes of modeling tools (e.g., quantum chemical, or molecular dynamics, or Potts model), establishing general practices across all tools, and then outlining procedures and expectations for specific classes of tools with partial inventories of codes that are examples of a given class of modeling tools. This Milestone, being cross-cutting in its impact, will be completed jointly with the FMM program element.

\subsubsection{Upscaling with Propagating Uncertainties}

Upscaling from sub-continuum scale into continuum models remains a scientific challenge for glass dissolution and other sub-continuum phenomena pertinent to the Waste IPSC. The identification of relevant phenomena at given scale, and the formulation and parameterization of effective abstracted models from those processes that can be used at subsequent scales, are, in general, unsolved problems which must be defined on a case-by-case basis. An additional requirement is that, once a physically-based abstraction is established, uncertainties must be propagated through the upscaling, simultaneously incorporating uncertainties from the form of the abstraction and the parameters that represent a realization of that abstraction. This propagation of uncertainties through the upscaling is a gap in the current state of knowledge. Collaborative work with the VU program element will develop and implement protocols for propagating UQ across boundaries, concurrent with the scientific upscaling procedures being developed.

\subsubsection{Validation Issues}

Capability development within the Waste IPSC will require data specifically targeted to model development and validation, and there is generally inadequate data for model development activities. The scope of NEAMS and the Waste IPSC does not include generating or commissioning experimental studies. Experimental data to satisfy validation requirements will require interaction with a robust experimental program coordinated with NEAMS efforts. The Waste IPSC will actively engage the DOE-NE Waste Form and UFD Campaigns to identify 
appropriate contacts and coordinate activities for those needs that are not satisfied through existing sources.

\subsubsection{Evidence Management}

All data used and generated by sub-continuum scale activities - verification and validation evidence in support of simulations, computational model constructions, etc. - needs to be documented and tracked. This record must be sufficiently complete to permit traceability and reproducibility, and include data and evidence needed to establish quantitative credibility. Current evidence tracking is ad hoc and incomplete. A needed facility is a system to track V\&V and UQ for the Waste IPSC. The requirements for an evidence management system were described in the Waste IPSC V\&V Plan (Edwards, et al. 2010).

\subsection{Summary of Glass Waste Form Dissolution Modeling}

A wide range of techniques available for modeling the dissolution of solid phases (primarily for minerals but also applicable to glasses) at the atomistic level provide opportunities to seamlessly link (upscale) these models with those at the continuum level. At the continuum level, recognition of the complex couplings in glass degradation and observation of the slow degradation of glass in geologic systems provide both the substance and opportunity to develop models that can demonstrate larger degrees of safety and confidence in disposal strategies applied over geologic time. Given these opportunities, a number of key gaps in understanding and capabilities have been identified. Recommendations for further work in these areas to enhance the ability to upscale models from the atomistic level to the high-fidelity continuum scale and into PA-scale models are provided here.

i. Calculate the protonation and deprotonation of different types of surface sites on glass surfaces using ab initio molecular dynamics calculations. Use Ab initio MD calculations to investigate the protonation and deprotonation of different types of surface sites on glass surfaces. These $a b$ initio MD calculations would need to be performed in combination with potentiometric titrations, vibrational and NMR spectroscopies, and neutron scattering to fully study the glass-water interface.

ii. The use of ab initio molecular dynamics to calculate reaction paths and activation energy barriers for the hydrolysis of bridging oxygen bonds may improve upon the use of molecular clusters. This approach would allow the calculation of reaction paths that invoke the cooperative behavior of multiple water molecules in the dissolution process.

iii. Parametric upscaling from atomistic to continuum processes. There are two study areas that have been identified as useful pursuits for this purpose. The first study area is to perform explicit atomistic models of glass dissolution to reproduce both rates and activation energies of the glass dissolution reactions. This will be approached initially by modeling feldspar dissolution as a simplified case to serve as an idealized proxy for glass dissolution (see discussion in Section 3.3.5). The second study area is to perform explicit atomistic models of the silica diffusion in the aqueous solution away from the glass surface (within the gel layer) to begin constraining the transport parameters more 
directly, as these are generally used as fitting parameters in current continuum models. For upscaling purposes, parametric extraction for continuum processes from experiments should be performed with a narrow focus on the stage of the glass degradation experiment so that it can be accomplished in a manner consistent with the dominant rate limiting process(es).

iv. Definition of the equilibrium phase for glass dissolution and correlations among glass rate parameters. Because glass is a thermodynamically unstable phase, choosing the appropriate representative composition for defining the equilibrium condition for use in a chemical affinity approach to the dissolution rate has been handled in various empirical ways in the literature (e.g., Verney-Carron et al. 2010). Development of a more generally applicable equilibrium reference point that accounts for variable glass composition would provide a more coherent framework for upscaling studies (e.g., use a standard set of overall reaction products that represent the stable assemblage of the glass composition). Correlation development between the rates of reactions and the free energy of overall reactions would also facilitate estimates of parameter values to be used for initial model construction and to delineate targeted experiments to measure confirmatory values.

v. Aqueous composition evolution at the reactive interface. Once the degradation process has progressed to the Evolution Stage, the pore fluid in the gel layer that is in contact with the glass reactive interface is no longer the same as the bulk solution composition. A number of studies have considered the changes in dissolved silica but this should be expanded to include full water compositional evolution in the pore fluid in the gel layer that is in contact with the glass reactive interface. Evaluating explicitly the changes at this location would be facilitated by a pore-scale reactive transport approach that included the major dissolved constituents.

vi. Surface area evolution of the reactive interface. In addition to the chemical changes that occur at the glass reactive interface as driven by gel layer development, physical occlusion of the reactive interface may reduce the overall mass of glass that is reacting with the fluids in the pores of the gel layer. Explicit delineation of these processes would facilitate understanding of how to apply such changes over time in a repository setting and would aid in consistent parameter extraction from results of experiments. Evaluating explicitly these physical changes at the reactive interface would be facilitated by a continuum-scale reactive transport approach.

vii. Field-scale progression of active surfaces contributing to the reactive interface total surface area using sub-continuum and continuum reformulations. In addition to changes in reactive or effective surface area at the glass-water interface due to an evolving gel layer structure, the effective surface area may increase dramatically as cracks in the glass surface nucleate and grow through time. Advances in continuum and sub-continuum understanding present an opportunity for a coherent upscaling study for crack development. The physical and chemical processes leading to decreased transport rate along altering cracks represent another area that may be amenable to upscaling analysis from sub-continuum to the continuum scale models. Additionally, developing general constraints on the integrated result of these field scale coupled processes (the occlusion of 
glass reactive interface surface area and additional crack growth) would address one of the major areas for upscaling from continuum models to field scale models. Evaluating explicitly these integrated physical changes to the total glass surface area that is available for reaction in the field would be facilitated by a continuum-scale reactive transport approach applied at the tens of centimeters to meter scale.

viii. Procedures to implement appropriate levels of verification and validation protocols need to be defined throughout all sub-continuum activities. Methods to evaluate and propagate quantitatively meaningful uncertainties through upscaling need to be developed and applied to ongoing model development activities, as those activities develop the scientific capabilities. 


\section{MODELING AND SIMULATION OF CONTINUUM PROCESSES}

This section describes progress in identifying applicable codes, and associated gaps, for the highfidelity-scale and PA-scale continuum models. Coupled multiphysics for THCMBR models combine conservation laws for mass, momentum and energy, together with phenomenological or experimentally based equations of state, kinematic conditions, transport laws, rate expressions, and other constitutive relations that express the linkages or couplings between processes. Gaps can exist in the necessary phenomenological expressions, the degree of coupling necessary between multiphysics, or in the numerical methods used for solution.

As described in Section 2, the FY10 gap analysis of code capabilities for Waste IPSC continuum processes, for both high-fidelity-scale and PA-scale models, was focused on four high-level THCM phenomena: EBS Environment, EBS Transport, Geosphere Environment, and Geosphere Transport. For the purposes of gap analysis, code capabilities were evaluated with respect to thermal-hydrologic-chemical (THC) phenomena and mechanical (M) phenomena. Within each of these gap analyses (THC and M), code capabilities to simulate the four high-level phenomena were considered collectively (e.g., the processes for EBS transport and geosphere transport are not significantly different). Additionally, each of the gap analyses considered code capabilities to simulate phenomena relevant to saturated hard rock, clay/shale, and salt geologic settings.

Section 4.1 describes relevant processes, equations, and methods for THC modeling, Section 4.2 present processes and equations for mechanical modeling, and Section 4.3 provides a preliminary gap analysis for THCM modeling.

\subsection{Thermal-Hydrologic-Chemical Processes and Code Capabilities}

Conservation or continuity relations for mass or energy density of a component $i$ in a phase $j$ can be written generally as (e.g., Martinez and Stone 2008):

$$
\frac{\partial d_{j}^{i}}{\partial t}+\vec{\nabla} \bullet \overrightarrow{\mathbf{q}}_{\mathbf{j}}^{\mathbf{i}}-Q_{j}^{i}
$$

where $\mathrm{d}$ is the mass or energy density, $\mathrm{q}$ is the flux density with respect to a reference coordinate system, and Q is a source term. Section 4.1.1 discusses applications of Equation 4.1.1 to thermal modeling; Section 4.1.2 describes hydrologic modeling and thermal-hydrologic couplings as well as applicable codes. Section 4.1.3 discusses chemical and reactive transport modeling and codes.

\subsubsection{Thermal Modeling}

Thermal modeling stemming from Equation 4.1.1 can be treated in a strict thermodynamic sense (with internal energy as the conserved variable) or, with assumptions, temperature can be assumed to be the conserved variable for heat transport. In the first case, a general expression for multiphase deformable fractured or porous media with saturations $S$ is (e.g., Martinez et al. 2001): 


$$
\frac{\partial}{\partial t}\left[(1-\phi) \rho_{s} e_{s}+\phi\left(\rho_{l} S_{l} e_{l}+\rho_{g} S_{g} e_{g}\right)\right]+\vec{\nabla} \bullet \overrightarrow{\mathbf{q}}_{e}=Q_{e}
$$

where $\phi$ is porosity, $\rho$ is density, $e$ is internal energy, and the subscripts $s, l$ and $g$ refer to solid, liquid, and gas respectively. The heat flux vector q can include terms for heat conduction, convection, and heat transport. The source term $Q$ can include heats of reaction, condensation and evaporation, and radioactive decay.

Common codes for heat transport in porous materials are discussed in the next section, as they are relevant to both heat and fluid flow.

\subsubsection{Hydrologic Modeling}

Similar to Equation 4.1.2, conservation laws can be written for aqueous, gaseous, and supercritical fluids (solutions) occupying intergranular pores in a deformable porous media. This requires specifying the flux density $\mathrm{q}$ in Equation 4.1.1 in terms of a fluid flux relative to the moving solid, and a solid phase velocity. The resulting terms couple directly to mechanics considerations of stress and deformation discussed in Section 4.2, such as solid phase compressibility, and elastic or elastic plastic deformation of the porous media. This is discussed in detail by Martinez and Stone (2008); Rutqvist et al. (2002) and Lo et al. (2002) among others. Ignoring these terms for simplicity, mass transport equations can be written for components $\mathrm{i}$ (here in the form of mass fractions $Y$ ) in the intergranular phases as:

$$
\frac{\partial}{\partial t} \phi\left(Y_{i l} \rho_{l} S_{l}+Y_{i g} \rho_{g} S_{g}\right)+\vec{\nabla} \bullet\left(Y_{i l} \rho_{l} \overrightarrow{\mathbf{v}}_{l}+Y_{i g} \rho_{g} \overrightarrow{\mathbf{v}}_{g}-\overrightarrow{\mathbf{J}}_{i g}\right)=Q_{i}
$$

Here $\mathrm{J}_{\text {ig }}$ is the gas phase flux, which can include multicomponent diffusion. The Darcy flux $\mathrm{v}$ for phase $\mathrm{j}$ is given by (Martinez et al. 2001):

$$
\overrightarrow{\mathbf{v}}_{j}=-\frac{k_{r j}}{\mu_{j}} k \bullet\left(\vec{\nabla} P_{j}+\rho_{j} \overrightarrow{\mathbf{g}}\right)
$$

where $k_{r}$ are the relative permeability for phases $\mathrm{j}, \mu$ is viscosity, $\mathrm{k}$ is the permeability tensor, and intrinsic property of the porous media, $P$ is pressure and $\mathrm{g}$ is gravitational acceleration. The set of Equations 4.1 .3 and 4.1.4 are supplemented by expressions relating capillary pressure $P_{c}=P_{g}-P_{l}$ and relative permeability as a function of saturations. There are numerous models for these (see for example Pruess 1999 or Martinez et al. 2001); a popular formulation for $P_{c}$ is developed by van Genuchten (1980). Equations 4.1.3 and 4.1.4 can be cast in a variety of forms, depending on the choice of primary variables (i.e. saturations, densities or pressures).

Multiphysics codes with relevant thermal-hydrologic capabilities include TOUGH2 (Pruess 1999), STOMP (White and Oostrom 2006), PorSalsa (Martinez et al. 2001), FEHM (Dash 2006), and SIERRA (Edwards 2002). These codes as they pertain to the Waste IPSC are discussed in Section 4.3 and Appendix A. 


\subsubsection{Multicomponent Multiphase Reactive-Transport Modeling}

This section briefly overviews the variety and structure of geochemical reactive transport codes in common practice today. It addresses capabilities currently in existence and under development for an advanced modeling and simulation program considered here.

Chemical modeling for subsurface systems needs to include a lengthy list of processes including aqueous speciation, dissolution/precipitation, redox processes (as mediated by microbiological processes), ion-exchange between solutions and minerals, surface chemical reactions occurring at phase interfaces (i.e. surface complexation, sorption), the effects of these processes on porosity and permeability, coupling with mechanical effects (e.g. water-assisted creep and crack growth; fracture healing, clay mineral swelling) as well as transport (advective, dispersive and multicomponent diffusion, osmotic potentials) and multiphase flow and reaction. We exclude from this synopsis MD modeling at interfaces and within solution phases, and pore scale (i.e. lattice Boltzmann methods) and focus instead on spatio-temporal continuum scale approaches. Our focus is on treatment of reacting porous media, and so do not discuss important aspects of transport as multiphase systems, heavy-tailed dispersion, or fractured porous media. Recent reviews on reactive transport modeling are by Crawford (1999), Steefel et al. (2005) and MacQuarrie and Mayer (2005).

Geochemical reaction transport models can be classed variously as inverse or forward; batch or transport; equilibrium, non-equilibrium, or "partial local equilibrium". All reaction-transport codes in use today for multicomponent spatial-temporal modeling use the partial local equilibrium approach, wherein homogeneous reactions such as aqueous speciation (most of which occur with rates faster than milliseconds (Brezonik 1994)) are taken at equilibrium, and heterogeneous reactions such as mineral-water dissolution/precipitation are kinetically mediated. This is by no means exclusive; for example, rates of aqueous conversion rates of gas phases like hydrogen, carbon dioxide or methane can occur on the time scale of hours. Biogeochemical cycling of sulfur in the aqueous phase at methane/brine seeps involves microbially-mediated homogeneous reactions that can occur on a similar time scale (Senko et al. 2005).

One source of debate is the question of metastability and the role of kinetics in subsurface processes. No one argues that diamonds occur metastably at surface conditions (the stable equilibrium phase under surface conditions would be graphite; diamonds persist due to large activation energy barriers). Some would argue that clay minerals, for example, are metastable precursors to equilibrium phyllosilicates such as micas. Important clay mineral transformations in the subsurface such as smectite-illite are interpreted in the context of the Ostwald step-rule (Morse and Casey 1988). Other workers assign thermodynamic properties to clays as distinct phases, with mixed results. These separate views impact modeling methodologies, i.e. local equilibrium versus a kinetic approach.

Thus users of a multicomponent, multiphase geochemical simulator need keep several questions in mind - what are the time and length scales under consideration; what is reactive buffering capacity (e.g. of gases and minerals); what are limitations on thermodynamic and kinetic data for the system in question; what are the options for model validation; what are the (geo) chemical 
and biological processes to include; and most importantly, what can be excluded for consideration.

\section{Speciation/Solubility Modeling}

At its simplest, geochemical modeling of multicomponent systems calculates the speciation of an interstitial solution at equilibrium and determines the saturation state of a suite of minerals and/or gases with respect to that solution. In other words, given an analytical suite of concentrations of elements, so-called speciation/solubility codes distribute moles or masses of the elements amongst discrete chemical species existing in solution at equilibrium at the temperature, pressure, and chemical conditions of interest. The following outlines this procedure, which is also used in reaction/transport models to update solution speciation and determine other solution properties.

Any chemical reaction mechanism involving $N$ species $A_{i}$ and $N_{r}$ chemical reactions can be expressed as:

$$
0=\sum_{l=1}^{N} v_{l r} A_{l}, \quad\left(r=1, \ldots ., N_{r}\right)
$$

where the $v$ are the stoichiometric coefficients, understood to be $<0$ for reactant species and $>0$ for product species. In all geochemical models, a discernment is made between basis species (the minimum set of species required to describe all species in a phase such as an aqueous solution, gas, or mineral). These are termed by various authors as "master", "component" or "primary" species and need not necessarily reflect actual species (Reed 1982). All chemical models (e.g., Wolery 1979) use an alternative description wherein all reactions involving secondary species (aqueous complexes) are written as (termed the canonical form; here we follow notation given in Lichtner (1996)):

$$
A_{i} \Leftrightarrow \sum_{j=1}^{N_{c}} \widetilde{v}_{j i} A_{j}
$$

Here, the new stoichiometric matrix is found from:

$$
\widetilde{v}_{j i}=-\sum_{r=1}^{N_{R}} v_{j r}\left(v^{-1}\right)_{r i}\left(j=1, \ldots, N_{c}\right) ;\left(i=N_{c}+1, \ldots ., N\right)
$$

where $N_{c}$ is the number of primary species $\left(N_{c}=N-N_{R}\right)$. Various geochemical databases express reaction sets in a given canonical form which is beneficial for retrieval, but also this form is necessary for solution. So-called speciation/solubility codes all involve a choice of components, a reduction of the system of equations to a minimum number (and a method of selecting stable mineral and gas phases at the conditions of interest, applicable to heterogeneous equilibria as in the next section). These models solve a set of nonlinear algebraic conditions including $\mathrm{N}_{\mathrm{c}}$ mass balance constraints, and $\mathrm{N}_{\mathrm{R}}$ mass action equations for the secondary species $\mathrm{i}$, which take the following general form (Reed 1982): 
Mass-balance:

$$
M_{\substack{i \\ \text { tot }}}^{\text {to }}=n_{W}\left[m_{i}+\sum_{i} \widetilde{\mho}_{j i} m_{i}\right]
$$

Mass action:

$$
K_{i}=\frac{\prod_{j} m_{j}^{\widetilde{\nu}_{j i}} \gamma_{j}^{\widetilde{j}_{j i}}}{m_{i} \gamma_{i}}
$$

Here $M^{\text {tot }}$ is total number of moles, $n_{w}=$ number of kilograms of solvent phase, $m_{i}$ is the molality of the $i^{\text {th }}$ species, and $\gamma_{i}$ is the activity or fugacity coefficient of the $i^{\text {th }}$ species in solution. Substituting the mass action equations into the mass balance equations yields a set of $N_{c}$ equations that be solved directly to determine the equilibrium state in terms of components. These are substituted back into the mass balance expressions to obtain the set of $\mathrm{N}$ species concentrations.

For aqueous solutions, the activity coefficients, reflecting the difference between activities (a thermodynamic model quantity) and concentrations (actual entities in a solution, at least in some statistical sense), are found from two distinct methods (see Bethke 1996 for a concise summary). In part these differences reflect parallel views of aqueous electrolyte solutions as being completely dissociated, partly dissociated, totally associated, or ignoring disassociation altogether. The first apply some form of the Debye-Hückel equation that takes into account long range ion interactions, and is dependent on the solution's ionic strength, ionic radii, and dielectric properties of the solvent. These include the original and extended Debye-Hückel equations, the Davies equation, and the B-dot model, variously applicable to solutions of increasing salinity or ionic strength, defined as:

$$
I=\frac{1}{2} \sum_{i} m_{i} z_{i}^{2}
$$

where $\mathrm{m}$ is concentration in molality and $\mathrm{z}$ is ionic charge. The B-dot method is routinely used to calculate activity coefficients for solutions of ionic strength up to a few molal, or just slightly more concentrated than seawater. Care must be taken when applying models for activity coefficients to not mix activity coefficients derived from different conventions and assumptions, and not to confuse models that use "true" ionic strength, which accounts for complexation reactions, and stoichiometric ionic strength, which assumes complete dissociation.

The second method can be termed the virial coefficient method, or "Pitzer" method (after the chemist who largely championed the method). This method is different from the Debye-Hückel methods in that little or no speciation of the solution need be accounted for, although some complexation is used especially for solutions at increasing temperature. In part this is because short range interactions become prominent as a solution increases in ionic strength, so what constitutes an actual species becomes blurred. As such this method is exclusively used for solutions at high ionic strength. These would include any aqueous solutions in salt repositories and in contact with crushed salt back-fill, most oil field waters, waters deriving from evaporation 
or boiling, waters in pore spaces that "dry out" as may occur during heating or in disturbed zones surrounding tunnels, and groundwaters in contact with evaporative minerals. There is much attention paid to expanding the range of applicability of Pitzer-type models to increasingly more complex solutions in the fields of nuclear-waste performance assessment, oil field reservoir quality, and subsurface carbon storage (e.g., Jove-colon et al. 2005).

Returning to speciation calculations, as the nonlinear set of mass balance and mass action equations depend on activity coefficients, and activity coefficients depend on solution speciation, these sets of equations must be solved iteratively; most use some form of Newton-Raphson method.

\section{Reactive Transport Modeling}

\section{Conservation of Mass}

There are many excellent publications devoted to spatio-temporal reaction-transport modeling. Below we outline a general treatment given by Lichtner (1996) and adopt his notation.

In general, conservation of mass for the $i^{\text {th }}$ aqueous or gaseous solute mass (in moles/volume) in a phase $\pi$ with saturation $s$ and porosity $\phi$ is given by:

$$
\frac{\partial}{\partial t}\left(\phi s_{\pi_{i}} c_{i}\right)+\vec{\nabla} \cdot \vec{J}_{i}=\sum_{r=1}^{N_{r}} v_{i r} I_{r} \quad(i=1, \ldots N)
$$

with flux $J$. The sum on the right hand side is over the total possible $N_{r}$ homogeneous and heterogeneous reactions $I_{r}$ in $\pi$, where $v_{i r}$ are the stoichiometric coefficients (number of moles of $i$ participating in the $r$ th reaction). Application to modeling chemical reaction and transport would require simultaneous numerical solution of $N$ partial differential equations (PDEs).

In practice this is a difficult task for several reasons including the lack of knowledge of aqueous speciation kinetics; the disparate time scale between homogeneous and heterogeneous reaction kinetics; and the orders of magnitude difference in the molar densities of solid and aqueous species (leading to "stiff" solutions and slow time stepping). Because of this, models for multicomponent reaction-transport all make certain simplifying assumptions that reduce the number of PDE's or otherwise simplify the numerical solution. Below we summarize these, and briefly discuss methods of solution in current use.

\section{Local Equilibrium Assumption}

One simplification that is in use (if not commonly) is to assume local equilibrium. Kirkner and Reeves (1988a; 1988b) discuss several different methods for transport and reaction assuming local equilibrium. Analogous to the batch equilibria treatment, these authors separate aqueous (and gaseous) species between primary (component) and secondary species, and also include sorption and mineral precipitation/dissolution. While this is strictly for a single phase pore fluid, the method can be extended to include additional pore phases. Letting $L$ represent the transport operator, their equations are: 


$$
\begin{gathered}
\frac{\partial \phi c_{j}}{\partial t}+L\left(c_{j}\right)=r_{j}^{c} \quad j=1, \ldots . ., N_{c} \\
\frac{\partial \phi x i}{\partial t}+L\left(x_{i}\right)=r_{j}^{x} \quad i=1, \ldots . ., N_{x}
\end{gathered}
$$

where $c_{j}$ is component $\mathrm{j}$ concentration, $x_{i}$ is secondary species $\mathrm{i}$ concentration, and the $\mathrm{r}$ are reaction rates where (aq denotes aqueous reactions, $\mathrm{c}$ denotes sorption, and $\mathrm{p}$ denotes precipitation)

$$
r_{j}^{c}=r_{j}^{c(a q)}+r_{j}^{c(s)}+r_{j}^{c(p)}
$$

Kirkner and Reeves (1988) treatment continues by specifying the $x$ in terms of the $c$ in canonical form:

$$
x_{i}=\gamma_{i}^{-1} K_{i} \prod_{j=1}^{N_{c}} a_{j}^{\widetilde{i}_{i j}} \quad\left(i=1, \ldots, N_{x}\right)
$$

and defining a total species concentration $u_{j}$ in terms of the $c$ and $x$ :

$$
u_{j}=c_{j}+\sum_{i=1}^{N_{x}} \widetilde{v}_{i j} x_{i}
$$

Multiplying Equation 4.1.13 by the stoichiometric matrix and summing over i to $N_{x}$ allows one to eliminate the homogeneous reaction rates, and to express reaction and transport in terms of the $u_{j}$ to obtain:

$$
\frac{\partial}{\partial t}\left(\phi u_{j}\right)+\frac{\partial}{\partial t}\left(\phi f_{j}^{s u}\right)+\sum_{k=1}^{N_{p}} B_{k j} \frac{\partial}{\partial t}\left(\phi p_{k}\right)+L\left(u_{j}\right)=0 \quad j=1, \ldots, N_{c}
$$

Here the $f$ are functions resulting from expressing the sorbed concentrations $s$ in terms of the $u$ (nonlinear mass-action expressions for equilibrium between solution concentrations and surface complexes), $B$ is a stoichiometric matrix for mineral dissolution/precipitation reactions, the $\mathrm{p}$ are moles of precipitate per volume of pore fluid, and $\mathrm{Np}$ is the number of solid phases. This method has reduced the number of unknowns from $2 N_{c}+N_{x}+N_{p}$ to $N_{c}$ PDE's plus $N_{p}$ mass action equations (relating $p_{k}$ to the $u_{j}$ ) (in reality, one would likely need to include $N_{c}$ additional equations to solve for the sorption terms).

Kirkner and Reeves (1988) also suggest an alternative method (first introduced apparently by Walsh et al. 1984) by using the total concentration of component $\mathrm{j}$ (aqueous + sorbed + solid), $w_{j}$, as:

$$
w_{j}=u_{j}+s_{j}+\sum_{k=1}^{N_{p}} B_{k j} p_{k} \quad j=1, \ldots, N_{c}
$$


in which case Equation 4.1.17 becomes:

$$
\frac{\partial}{\partial t}\left(\phi w_{j}\right)+L\left[f_{i}^{u w}\left(w_{1}, \ldots, w_{N_{c}}\right)\right]=0 \quad j=1, \ldots, N_{c}
$$

a system of $N_{c}$ PDE's, $N_{p}+N_{x}$ mass action equations, and $N_{c}$ equations of the form of Equation 4.1.18. The algebraic expressions are directly related to the batch equilibrium relations in Equations 4.1.8 and 4.1.9, and so the method basically consists of solving batch equilibrium at every node coupled to a transport equation (Equation 4.1.19). The local equilibrium assumption should be used when the transport time given a length scale of interest is much longer than the time scale for equilibration (Steefel and Van Cappellan 1990).

\section{Local Partial Equilibrium Assumption}

The previous time-scale issues do not hold for many problems of interest and so many surface and subsurface chemical problems are critically dependent on the kinetics of reactions. Widely popular is the assumption of local partial equilibrium, or fast and slow reactions (Lichtner 1996), in which fast reactions like most homogeneous reactions are taken at equilibrium, while slow reactions (including many mineral-water reactions) are kinetically mediated (Lichtner 1985; Ortoleva et al. 1986; 1987a; 1987b; Yeh and Tripathi 1989; 1991). This method is covered in detail in the summary text by Lichtner et al. (1996) and follows much of the derivation in the previous section. In the case where all mineral reactions are treated kinetically, and where mass diffusion of components is species-independent, Lichtner (1996) shows that a description of local partial equilibrium involving mineral-solution reactions is expressed as:

$$
\begin{gathered}
\frac{\partial}{\partial t}\left(\phi \Psi_{j}\right)+\vec{\nabla} \cdot \vec{\Omega}_{j}=-\sum_{m=1}^{N_{\min }} \widetilde{v}_{j m} I_{m}+\sum_{r=1}^{N_{R}^{k e}} v_{j r}^{\prime} I_{r}^{k e} \\
\frac{\partial \phi_{m}}{\partial t}=\bar{V}_{m} I_{m}
\end{gathered}
$$

where:

$$
\begin{aligned}
& \Psi_{j}=c_{j}+\sum_{i=1}^{N_{a q}} \widetilde{v}_{j i} c_{i} \\
& \Omega_{j}=(-\tau \phi D \vec{\nabla}+\vec{q}) \Psi_{j} \\
& v_{j r}^{\prime}=v_{j r}^{k e}+\sum_{i=N_{c}^{l e}+1}^{N_{l e}} \widetilde{v}_{j i} v_{i r}^{k e}
\end{aligned}
$$

$N_{a q}$ is the number of aqueous species, $N_{\min }$ is the number of minerals, $N_{R}{ }^{k e}$ is the number of kinetically-controlled reactions, $N_{l e}$ is the number of reactions at local equilibrium (i.e. 
complexation reactions), $\mathrm{N}_{\mathrm{c}}^{\text {le }}$ is the number of components or primary species, the $I$ are reaction rates, $V_{m}$ is the molar volume of solid $m$, and $\phi_{m}$ is the mass fraction of solid $m$.

So-called mineral rate laws give the reaction rate in terms of reactive surface area and solution and sorbed species concentrations. At the very least these are generally nonlinear functions of the primary species concentrations $c_{j}$. A review of mineral-water reaction rate laws and associated parameters is found in Palandri and Kharaka (2004). Also of interest for coupling to geomechanical codes, in an Eulerian frame, Equations 4.1.20 and 4.1.21 can be modifiable to account for solid matrix deformation (Dewers and Ortoleva 1990).

One problem with the above approach is that in many subsurface systems, mineral-water reaction kinetics for common mineral types occurs on widely disparate time scales (e.g. carbonates and sulfates equilibrate with aqueous solutions on the order of days or months at surface conditions, while silicates can take tens to hundreds of thousands of years). Ranges in reaction rate time scales can produce very stiff numerical solutions.

\section{Quasi-Stationary State}

For a particular system, if the time scale for substantial reaction involving a solid phase is orders of magnitude greater than the time scale for solute reaction and transport to reach a steady state, then a viable approximation for the reaction transport equations (i.e. Equation 4.1.20) is the quasi-stationary state approximation (Ortoleva et al. 1986; 1987; Lichtner 1988; Dewers and Ortoleva 1990; 1992) which amounts to ignoring the time derivative in Equations 4.1 .11 and 4.1.20. This approach is valid for long (geologic) time scales and has been shown useful in describing zonation associated with reaction fronts and chemical waves, and in coupled mechanical-chemical formulations describing basin evolution and rock lithification patterns.

\section{First-Order Decay and Retardation}

A simplified reactive transport formulation for a radionuclide species with first order decay and adsorptive retardation can be set forth as follows (e.g., Hansen et al. 2010, p. 44). Conservation of mass for the $i^{\text {th }}$ aqueous or gaseous solute mass ( $c_{i}$ is the molar concentration) in a phase $\pi$ with saturation $s$ and porosity $\phi$ is given from Equation 4.1 .17 by:

$$
\frac{\partial}{\partial t}\left(\phi s_{\pi_{i}} c_{i}\right)+\vec{\nabla} \cdot \vec{J}_{i}=\sum_{r=1}^{N_{r}} v_{i r} I_{r} \quad(i=1, \ldots N)
$$

The sum on the right hand side is over the total possible $N_{r}$ homogeneous and heterogeneous reactions $I_{r}$ in $\pi$, where $v_{i r}$ are the stoichiometric coefficients (number of moles of $i$ participating in the $r$ th reaction, Lichtner 1996; see Martinez et al. 2001 for similar treatment). We consider only a single solute species, c, in a liquid phase, and account for advective and diffusive flux, wherein Equation 4.1.25 becomes:

$$
\frac{\partial}{\partial t}\left(\phi s_{L} c\right)+\vec{\nabla} \cdot\left(\vec{v}_{L} c\right)=\vec{\nabla} \cdot\left(\phi s_{L} D \vec{\nabla} c\right)+\phi R
$$


Here $\mathrm{v}_{\mathrm{L}}$ is the liquid Darcy velocity and $\mathrm{D}$ is an effective mass diffusion coefficient which includes a tortuosity, i.e. $\mathrm{D}=\mathrm{D}_{\mathrm{m}} \tau$. $\mathrm{R}$ is the net molar production rate of $\mathrm{c}$, which accounts both for first order radioactive decay and a term accounting for sorption. Following the treatment by Schwartz and Zhang (2003, their Equation 23.12), Equation 4.1.26 becomes:

$$
\frac{\partial}{\partial t}\left(\phi s_{L} c\right)+\vec{\nabla} \cdot\left(\vec{v}_{L} c\right)+\frac{\partial}{\partial t}\left(s a_{m}\right)=\vec{\nabla} \cdot\left(\phi s_{L} D \vec{\nabla} c\right)+r
$$

The third term on the left-hand-side is the time rate of change of the product of an areal molar concentration $\mathrm{s}$ and the specific surface area of mineral per unit bulk volume, $\mathrm{a}_{\mathrm{m}}$ (Thomson and Jackson 1996), and $r$ accounts for any other chemical reaction rate.

When sorption reaction rates are considered rapid relative to transport rates, $\mathrm{s}$ will approach a local equilibrium with the local bulk fluid concentration $\mathrm{c}$, and thus can be represented by a sorption isotherm. Using a linear sorption isotherm, one defines the commonly used retardation factor $\mathrm{R}_{\mathrm{f}}$ in Equation 4.1.27:

$$
\frac{\partial}{\partial t}\left(\phi s_{L} c R_{f}\right)+\vec{\nabla} \cdot\left(\vec{v}_{L} c\right)=\vec{\nabla} \cdot\left(\phi s_{L} D \vec{\nabla} c\right)+r
$$

where $R_{f}=1+a_{m} k / \phi s_{L}$ with $k$ the isotherm constant. Usually retardation is defined in terms of an apparent distribution coefficient (as a means of relating sorption behavior to experimental measurement) $K_{d}$, which relates the total contaminant mass adsorbed per total solid mass to the bulk aqueous concentration (Tompson and Jackson 1996). With $K_{d}=a_{m} k / \rho_{b}$ with $\rho_{b}$ the bulk mass density, $R_{f}=1+\rho_{b} K_{d} / \phi s_{L}$, i.e. Equation 23.14 of Schwartz and Zhang (2003), here modified for partially saturated media.

Finally, for a solute species undergoing first order radioactive decay, $r=-\phi s_{L} R_{f} \lambda c$, where $\lambda$ is the decay constant, related to radionuclide half-life by $t_{1 / 2}=\ln (2) / \lambda$ (Equation 23.16 in Schwartz and Zhang 2003).

\section{Texture Distributions and Population Balance Approach}

The treatment of solid phase and pore texture in the above examples of systems of equations is seen to be based on "average" properties (porosities, volume fractions, mean crystal sizes) which is at best a crude description of a reacting porous media. While pore scale methods can treat such variations explicitly (as in lattice Boltzmann approaches), at the continuum scale a better description of a reacting porous media takes explicit account of mineral grain and pore-size distributions. Sometimes termed "particulate" or "population" dynamics, this approach has been popularized by chemical engineers (a classic text is by Randolph and Larson (1988)) applied to, e.g. industrial scale batch crystallization (Ramkrishna 2000), but is rarely used in treatments for subsurface reactive transport (Eberl et al. 1990; Steefel and Van Cappellan 1990; Dewers and Reches 2004). Including a complete size and/or pore distributions can be critical in accurately describing water-rock interaction as mineral grain sizes in the lower tail of a crystal size distribution may have minimal volume, but possess the bulk of reactive surface area. 
A population balance equation for crystal growth invokes the population distribution of crystal sizes or particulates L, n(L), as (Randolph and Larson 1988):

$$
\frac{\partial n}{\partial t}=\vec{\nabla} \bullet(n \vec{u})+B-D
$$

where $\mathrm{u}$ is the particulate velocity vector in "phase space" which includes spatial coordinates and non-spatial internal coordinates which can include particulate size. In this case u, for sizeindependent growth, is equal to the crystal growth rate. B and D are the so-called "birth" and "death" functions which can include physics such as nucleation, agglomeration, aggregation, fracturing and healing of grains, etc. Ostwald ripening, in which case the growth velocity depends on L or size-dependent growth, is a special case of Equation 4.1.29 (Eberl et al. 1990). Approaches like Equation 4.1.29 are common in studies of microbial population dynamics (for example, see Lauffenberger 1991).

A similar population equation could be constructed for porosity, e.g., and could include coupled physics like pore collapse through grain crushing, in which the pore size distribution and grain size distribution are coupled through ' $\mathrm{B}$ ' and ' $\mathrm{D}$ ' terms. Other examples of coupling include hydrologic - permeability is often as a function of the pore size distribution, and mechanical recent "creep laws" for geomaterials, for example, are often expressed as a function of the crystal size distribution. Combining the above within a full reactive transport methodology has rarely if ever been attempted (with time and three spatial dimensions, this would require numerical solutions in a five dimensional space and codes simply aren't set up for that). One simplification in common use is to express Equation 4.1.29 in terms of the moments of distribution. In this case, ignoring transport, the single PDE in Equation 4.1.29 is reduced to four coupled ordinary differential equations (ODEs) for particle number density, average size, surface area, and volume fraction.

Finally, population balance-like methods may be a viable alternative to upscaling methods like homogenization, in which case upscaled physics can include aspects like distribution taildependent physics, chemical microenvironments, and pore-scale heterogeneity, which are lost by averaging techniques.

\section{Other Relevant Physics}

Although a comprehensive review of reactive transport modeling in its entirety is beyond the scope of this treatment, other relevant physics require mentioning that play important roles in modeling reactive transport in high level nuclear waste disposal scenarios.

In concentrated electrolyte solutions, and other dense solutions such as melts, multicomponent transport, in which diffusional transport of a dissolved species is dependent not only on the electrolyte properties but also on the chemical gradients of other species. Treatises on multicomponent diffusion relevant to waste disposal in geologic settings are found in Appelo and Wersin (2007) and Appelo et al. (2010).

Surface reactions other than dissolution and precipitation figure prominently in radionuclide transport and can be directly coupled to continuum reactive transport treatments such as 
Equations 4.1.20 through 4.1.24. Older methods such as the $\mathrm{K}_{\mathrm{d}}$ and sorption isotherm approaches (such as the linear example in Equations 4.1.26 and 4.1.27) are being replaced by more sophisticated surface complexation models (Davis and Kent 1990; Goldberg et al. 2007). Although still new in development, surface complexation methods permit a better predictive capability as well as a better understanding on solution chemistry effects on reaction kinetics.

\section{Numerical Methods for Coupling Reaction and Transport}

A brief treatise on numerical methods applied to reactive transport modeling is relevant to this discussion. The simplest and most direct method, but not always the most numerically efficient and subject to convergence problems for especially non-linearly coupled sets of PDEs is the "One-step" or global implicit approach (Steefel and Lasaga 1994; Steefel and MacQuarrie 1996) - this involves solving a fully coupled system with a large Jacobian matrix for typical Newton method. Similar but newer and more efficient approaches use a modified Newton method (Steefel and MacQuarrie 1996). These include solving for mixed differential-algebraic equations solving for the $\psi_{i}$ or $u_{i}$ (Lichtner 1985) or direct substitution of mass action expressions into PDE's and solving for the $c_{j}$ (Steefel and Van Cappellan 1990). While this is the simplest and most direct approach, it can be slow. Thus many workers employ some sort of operator split which include:

- Sequential non-iterative approach (SNIA) in which a single time step consists of transport step followed by reaction step using transported equations; generally simple and robust but problems occur at boundaries; it also tends to overestimate reaction amounts in problems where concentrations are continually decreasing (i.e. decay reactions; Steefel and MacQuarrie 1996);

- Strang splitting - similar to SNIA but centers the reaction step in middle of two transport steps;

- Sequential iterative approach (SIA) (recommended by Yeh and Tripathi 1989; 1991); several schemes are in use but involves iterating between reaction and transport within a single time step. One method involves alternating reaction and transport terms as a source term from the previous iteration; this has shown convergence problems for some types of reaction schemes.

Jacobian-free Newton-Krylov methods are gaining popularity and need mentioning (Hammond et al. 2007, based on Hammond's thesis and work on core PFLOTRAN). Jacobian-free NewtonKrylov was developed to avoid the need for a Jacobian matrix, which is computationally expensive to compute and store. Jacobian-free Newton-Krylov involves computing the matrixvector products of a Krylov solver (e.g., conjugate gradient (CG), generalized minimum residual (GMRES), bi-conjugate gradient stabilized (Bi-CGSTAB), available in Sandia National Laboratories TRILINOS; Heroux et al. 2005) by employing a numerical approximation to the Jacobian-Krylov vector product. It can be computationally efficient in memory and processing. However, it requires specific preconditioning and a large number of Krylov solver iterations.

Relevant multiphysics codes for the Waste IPSC evaluations include TOUGHREACT (Xu et al. 2004), FEHM (Dash 2006), STOMP (White and Oostrom 2006) and SIERRA (Edwards 2002). 
These codes are discussed in Section 4.3 and Appendix A. Additional reactive transport codes are also listed in Appendix A.

\subsection{Mechanical Processes and Code Capabilities}

\subsubsection{Governing Mechanical Equations}

For mechanical (geo-mechanical) systems, there are three basic sets of equations that govern the description of a system deforming under a given load. The first set is the set of equations of motion:

$$
\sigma_{i j, j}+\rho b_{j}=\rho a_{j}
$$

or, for the case when the processes are very slow such that inertia $\left(\rho a_{j}\right)$, may be neglected, these equations become the equilibrium equations:

$$
\sigma_{i j, j}+f_{i}=0
$$

where $\sigma_{i j}$ are the components of the stress tensor and $f_{i}=\rho b_{i}$ are the body forces. The second set is the set of strain-displacement relations:

$$
e_{i j}=\frac{1}{2}\left(u_{i, j}+u_{j, i}+u_{k, j} u_{k, i}\right)
$$

where $e_{i j}$ is the strain tensor and $u_{i}$ is the displacement vector.

The third set of equations, the so-called constitutive equations, relates the equilibrium equations to the strain-displacement relations through the material (constituent) response of the material that is undergoing the deformations. This third set of equations can take on many forms depending on the material that is being modeled, ranging all the way from a simple elastic material that could be used to model, say a granitic material, to materials such as clay and rock salt, with significantly more complicated behaviors that require significantly more sophisticated and involved material descriptions.

As an example of this third set of equations, consider a constitutive model for the latter material above, rock salt (Krieg 1984). It is one of the materials of interest for Waste IPSC applications and is a creeping material with a creep rate that is highly temperature-dependent. Its overall strain rate can be characterized by the equation:

$$
\dot{e}_{i j}=-\frac{v}{E} \dot{\sigma}_{k k} \delta_{i j}+\frac{1+v}{E} \dot{\sigma}_{i j}+\dot{e}_{i j}^{C}+3 \alpha \dot{T} \delta_{i j}
$$

where $\sigma_{i j}$ are the components of the stress tensor, $v$ is the Poisson's ratio, $E$ is Young's Modulus, $T$ is temperature $\left({ }^{\circ} \mathrm{K}\right), \alpha$ is the coefficient of linear thermal expansion, and $\delta_{i j}$ is the Kronecker Delta. 
The creep strain rate, $\dot{e}_{i j}^{C}$, is given by:

$$
\dot{e}_{i j}^{C}=\left|\dot{e}_{k l}^{C}\right| \frac{\sigma^{\prime} i j}{\left|\sigma^{\prime} i j\right|}
$$

where $\sigma_{i j}^{\prime}$ are the components of the deviatoric stress tensor. If one takes the simplest constitutive model for rock salt mechanical behavior in which only secondary creep (steadystate) is considered, the magnitude of the creep rate can now be expressed in terms of the effective creep strain rate, $\dot{\bar{e}}$, or the effective stress, $\bar{\sigma}$, as follows (Hermann, et al. 1980):

$$
\begin{aligned}
& \left|\dot{e}_{i j}^{C}\right|=\sqrt{1.5} \dot{\overline{\mathrm{e}}} \\
& \dot{\overline{\mathrm{e}}}=D \bar{\sigma}^{n} \exp \left(-\frac{Q}{R T}\right),
\end{aligned}
$$

where $\dot{\overline{\mathrm{e}}}$ is defined as:

$$
\dot{\overline{\mathrm{e}}}=\left(\frac{2}{3} \dot{e}_{i j}^{C} \dot{e}_{i j}^{C}\right)^{1 / 2}
$$

while $\bar{\sigma}$ is:

$$
\bar{\sigma}=\left(\frac{3}{2} \sigma_{i j}^{\prime} \sigma_{i j}^{\prime}\right)^{1 / 2}
$$

$D$ and $n$ are constants determined from data analysis (typically from laboratory-scale creep tests), and $T$ is the temperature $\left({ }^{\circ} \mathrm{K}\right), Q$ is the effective activation energy (cal/mole), and $R$ is the universal gas constant $\left(1.987 \mathrm{cal} / \mathrm{mole}^{\circ}{ }^{\circ} \mathrm{K}\right)$.

While the secondary creep constitutive model for rock salt described above is a simple example of a basic model for rock salt, there are a variety of other constitutive models for rock salt that may be needed to better describe its response. Those could range from models like the Unified Creep-Plasticity model (Aubertin et al. 1991) to the Multi-mechanism Deformation (Munson, et al. 1989) and the Multi-mechanism Deformation Coupled Fracture (MDCF) (Chan et al. 1999) models of Munson and co-workers. The latter two models have been extensively used at the Waste Isolation Pilot Plant (WIPP) for years because they can model a wider range of rock salt behavior (including primary, secondary, and tertiary creep, for the case of the MDCF model).

In a similar vein and because there are various materials that may comprise different geologic repository settings, other constitutive models of interest for this application may be needed. These might include the following:

- clay materials models (e.g., Alonso, et al. 1990; Wheeler and Sivakumar 1995),

- plasticity models (e.g., Krieg 1978; Sjaardema and Krieg 1987) for the various components in the EBS, and, 
- other generalized cap-plasticity models (e.g., Fossum and Brannon 2004; Brannon et al. 2009) that could be used for a range of different geo-materials that may characterize repository geosphere.

\subsubsection{Mechanical Modeling}

The three sets of equations, described above, that govern mechanical (geomechanical) response can and have been implemented in various computer codes using different discretization and solution techniques. However, the most common implementations are those that use the finite element method as the discretization technique and an implicit solver as the solution technique. The resulting finite element codes themselves can range from:

- individual stand-alone applications for addressing a specific class of problems (e.g., implicit, small strain, linear elastic material response);

- to more general implementations typically derived for a broader class of problems such as is typical of many DOE codes aimed at the broader class of national laboratory applications, e.g., explicit, large deformation, inelastic material response (Lin 2005; Maker 1995; Taylor and Flanagan 1989; Blanford et al. 2001; SIERRA Mechanics Team 2010a; SIERRA Mechanics Team 2010b),

- to the most general implementation that addresses broad classes of problems such as is typical of commercial codes, e.g., implicit/explicit, small/large deformation, general material response (ANSYS 2010; LTSC 2003; SIMULIA 2007).

For the Waste IPSC, the mechanical code capability most likely to be of importance to address the broader range of waste forms, engineered barrier systems, and repository settings is the ability to model large strain/deformation. This is needed for the large deformations anticipated to occur in some disposal system settings. Similarly, a robust and efficient contacting surface capability will also be needed to complement these large deformations, as these may lead to surfaces that develop contact interactions as they deform toward each other, although originally they may not have been in contact with each other. Also needed is the ability to model general inelastic response, to be able to model the behavior of the various materials like rock salt, clay, crushed rock, etc., that might comprise the various disposal system components. Hence, a robust constitutive model interface will be needed to be able to supplement the material library that exists in a code.

\subsection{Preliminary Gap Analysis of THCM Code Capabilities}

A preliminary assessment of eight existing high-fidelity simulators for potential use in modeling coupled THCM processes has been performed. These are: TOUGHREACT, FEHM, STOMP, HYDROGECHEM, CANTERA, EQ3/6, DAKOTA, and SIERRA. It should be noted that this preliminary assessment was performed on the basis of limited available public information; consequently there may be other capability available in the codes that was not listed in those sources and hence is not included or acknowledged below. It is our intent that sometime in the near future we will acquire the various simulators and perform a more complete hands-on assessment that may change the outcome of our preliminary assessment of these. 
- TOUGHREACT is a numerical simulation program for chemically reactive non-isothermal flows of multiphase fluids in porous and fractured media. The program is written in Fortran 77 and was developed by introducing reactive chemistry into the multiphase flow code TOUGH2 V2. Interactions between mineral assemblages and fluids can occur under local equilibrium or kinetic rates. The gas phase can be chemically active. Precipitation and dissolution reactions can change formation porosity and permeability, and can also modify the unsaturated flow properties of the rock. The code is distributed with a comprehensive user's guide that includes sample problems addressing geothermal reservoirs and hydrothermal systems, nuclear waste isolation, groundwater quality, sequestration of carbon dioxide in saline aquifers, and supergene copper enrichment.

A wide range of subsurface thermo-physical-chemical processes can be considered under various thermo-hydrologic and geochemical conditions of pressure, temperature, water saturation, and ionic strength. The program can be applied to one-, two- or three-dimensional porous and fractured media with physical and chemical heterogeneity. The model can accommodate any number of chemical species present in liquid, gas and solid phases. A variety of equilibrium chemical reactions are considered, such as aqueous complexation, gas dissolution/exsolution, and cation exchange. Mineral dissolution/precipitation can proceed either subject to local equilibrium or kinetic conditions. Changes in porosity and permeability due to mineral dissolution and precipitation can be considered. Linear adsorption and decay can be included. For the purpose of future extensions, surface complexation by double layer model is coded in the program (Xu, et al. 2004; Pruess et al. 1999).

- FEHM (Finite Element Heat and Mass) is a computer code that simulates complex coupled subsurface processes as well as flow in large and geologically complex basins. FEHM is a numerical simulation code for subsurface transport processes. It models three-dimensional, time-dependent, multiphase, multicomponent, non-isothermal, reactive flow through porous and fractured media. It can represent complex geologic structures and their effects on subsurface flow and transport. FEHM has been used to simulate groundwater and contaminant flow and transport in deep and shallow, fractured and non-fractured porous media throughout the DOE complex. Its development has spanned several decades; a time over which the art and science of subsurface flow and transport simulation has dramatically evolved. Advanced model analysis requires a detailed understanding of model errors (numerical dispersion and truncation) as well as those associated with the application (conceptual and calibration) Application errors are evaluated through exploration of model and parameter sensitivities and uncertainties. The development of FEHM has been motivated by subsurface physics of applications and also by the requirements of model calibration, uncertainty quantification, and error analysis. FEHM possesses features and capabilities that are of general interest to the subsurface flow and transport community and it is well suited to hydrology, geothermal, and petroleum reservoir applications (Zyvoloski 2007; Dash 2006).

- STOMP (Subsurface Transport Over Multiple Phases) is a simulator that has been developed by the Pacific Northwest National Laboratory for modeling subsurface flow and transport systems and remediation technologies. The STOMP simulator's fundamental purpose is to produce numerical predictions of thermal and hydro-geologic flow and transport phenomena 
in variably saturated subsurface environments, which are contaminated with volatile or nonvolatile organic compounds. The STOMP simulator is written in the FORTRAN 77 and 90 languages, following American National Standards Institute (ANSI) standards. The simulator utilizes a variable source code configuration, which allows the execution memory and speed be tailored to the problem specifics, and essentially requires that the source code be assembled and compiled through a software maintenance utility. Auxiliary applications include numerical predictions of solute transport processes including reactive transport. Quantitative predictions from the STOMP simulator are generated from the numerical solution of PDEs that describe subsurface environment transport phenomena. Description of the contaminated subsurface environment is founded on governing conservation equations and constitutive functions. Governing coupled flow equations are PDEs for the conservation of water mass, air mass, $\mathrm{CO}_{2}$ mass, $\mathrm{CH}_{4}$ mass, volatile organic compound mass, salt mass, and thermal energy. Constitutive functions relate primary variables to secondary variables. Solution of the governing PDEs occurs by the integral volume finite difference method. The governing equations that describe thermal and hydrogeologic flow processes are solved simultaneously using Newton-Raphson iteration to resolve the non-linearities in the governing equations. Governing transport equations are PDEs for the conservation of solute mass. Solute mass conservation governing equations are solved sequentially, following the solution of the coupled flow equations (White and Oostrom 2006).

- HYDROGEOCHEM is a set of finite element based reactive transport codes developed by Professor G. Yeh and his students over the last 20 years at the Pennsylvania State University and Central Florida University (Yeh and Tripathi 1990; Yeh et al. 2004). HYDROGEOCHEM couples geochemical reactions to flow, heat transfer and mass transport processes in 2 and 3 dimensions. Over the past two decades, Sandia National Laboratories has been involved in several efforts to evaluate the use of the HYDROGEOCHEM family of codes. The family of HYDROGEOCHEM codes also includes several geochemical codes that do not include transport: (1) EQMOD: equilibrium geochemical speciation calculations that simulates aqueous complexation, surface adsorption, ion-exchange, precipitation/dissolution, and redox reactions; (2) KEMOD: A series of chemical speciation codes in which every chemical species can be treated as either an equilibrium-controlled or kinetically-controlled species. The later versions have increasingly greater flexibility in simulating kinetically controlled reactions; (3) EQPITZ: a geochemical equilibrium code based on EQMOD that uses the Pitzer equations to calculate activity coefficients up to $20 \mathrm{M}$ ionic strength; (4) EQMODCP: a geochemical equilibrium modeling code that can simulate coprecipitation, in addition to the processes represented in EQMOD; (5) BIOGEOCHEM: a chemical speciation code and a preprocessor for HYDROGEOCHEM simulations. This code is a descendant of the KEMOD code and includes additional features for simulation of microbial processes. It incorporates a new paradigm of reaction-based approaches to biogeochemical processes (Fang et al. 2003) which allows description of the reaction system on an ad hoc empirical basis or in terms of more fundamental basic reactions. The modular nature of the HYDROGEOCHEM code family is an attractive feature for Waste IPSC.

- CANTERA is a suite of object-oriented software tools for problems involving chemical kinetics, thermodynamics, and/or transport processes (http://code.google.com/p/cantera/). With Cantera's object technology, an architectural masterpiece (i.e., application program) can 
be assembled from a set of very special bricks picked up at the Cantera quarry. Each "brick" (or object) represents some well-defined small component of the global structure. Some of the types (or classes) of objects Cantera provides represent: phases of matter, interfaces between these phases, reaction managers, time-dependent or steady reactor networks, and one-dimensional flows. Some of the areas where Cantera is used currently include combustion, flames structure, detonations, electrochemical energy conversion and storage, fuel cells, and batteries. Cantera 2.0 will add many new classes of objects, enabling Cantera to be used for many new applications and enabling several exciting new ways to interface with matlab and python. It's currently in its formative stages and is not working.

- EQ3/6 is a chemical equilibrium code widely used in the geochemical community (Wolery and Jarek 2003). EQ3/6 performs two kinds of calculations pertaining to aqueous solutions and aqueous systems. The first kind is called a speciation-solubility calculation. Such a calculation is to describe the chemical and thermodynamic states of a solution for given chemical compositional constraints and equilibrium assumptions. The second kind of calculation is called a reaction-path calculation, which simulates the evolution of a chemical system as the overall reaction progress. EQ3/6 does not have capability for flow transport.

- DAKOTA (Design Analysis Kit for Optimization and Terascale Applications) is a software toolkit that provides a flexible and extensible interface between simulation codes and iterative analysis methods used in large-scale systems engineering optimization, uncertainty quantification, and sensitivity analysis (Eldred et al. 2002). Strictly speaking, DOKOTA is not a THCM code. However, it can be a good candidate as a performance assessment driver for THCM simulations (Wang et al. 2010). The DAKOTA toolkit can perform parameter optimization through the use of gradient and nongradient-based methods. It can also be used to conduct sensitivity analysis with the purpose of investigating variability in response to variations in model parameters using sampling methods such as Latin hypercube sampling (LHS), among others. Further capabilities of the toolkit include uncertainty quantification with sampling, analytic reliability, and stochastic finite element methods; and parameter estimation with nonlinear least squares methods. These capabilities may be used on their own or as components within system models. By employing object oriented design to implement abstractions of the key components required for iterative systems analyses, the DAKOTA toolkit provides a flexible and extensible problem-solving environment for design and performance analysis of computational models on high performance computers.

- SIERRA is a code suite built around a toolkit that provides code services and underpins the framework of the simulator. The development of the SIERRA Mechanics code suite has been funded by the DOE Advanced Simulation and Computing (ASC) program for over ten years. The goal is the development of massively parallel multiphysics capabilities to support the Sandia National Laboratories' engineering sciences mission. SIERRA Mechanics was designed and developed from its inception to run on the latest and most sophisticated massively parallel computing hardware; spanning the hardware compute space from a single workstation to compute systems with 1000's of processors. The foundation of SIERRA Mechanics is the SIERRA toolkit which provides finite element application code services such as: (1) mesh and field data management, both parallel and distributed, (2) transfer operators for mapping field variables from one mechanics application to another, (3) a 
solution controller for code coupling, and (4) included third party libraries (e.g. solver libraries, MPI communications package, etc.).

The SIERRA Mechanics code suite is comprised of application codes that address specific physics regimes. Included are thermal and fluid mechanics analysis codes; solid mechanics and structural dynamics codes; and software development tools used by the SIERRA Mechanics codes for configuration management, software builds, and release management. The two SIERRA Mechanics codes which are used in the THCM coupling are SIERRA/FD (Aria) and SIERRA/SM (Adagio). The suite of physics currently supported by Aria includes the incompressible Navier-Stokes equations; the energy transport equation; species transport equations; as well as generalized scalar, vector and tensor transport equations. A saturated porous flow capability is a recent addition to Aria, and multiphase porous flow is under development. Aria also has some basic geochemistry functionality available through existing chemistry packages such as Chemeq and Cantera. The solid mechanics portion of the THCM coupling is handled by Adagio which solves for the quasistatic, large deformation, large strain behavior of nonlinear solids in three-dimensions. Adagio has some discriminating technology that has been developed at Sandia for analyzing problems in solid mechanics. This technology involves the use of matrix-free iterative solution algorithms that allow extremely large and highly nonlinear problems to be solved efficiently. This technology also lends itself to effective and scalable implementation on massively parallel computers. The THCM coupling is done through a solution controller within SIERRA Mechanics called Arpeggio (Edwards 2002; Notz et al. 2007; SIERRA Mechanics Team 2010a; SIERRA Mechanics Team 2010b).

The codes described above appear to have significant capability in the THC coupled-physics realm, with capability of varying degrees in terms of reactive transport, from fledgling (SIERRA) to advanced (TOUGHREACT, HYDROGEOCHEM). However, there is an apparent lack of complete coupling of mechanical (M) capability in all but one of them (SIERRA). While two of the codes, TOUGHREACT and FEHM, appear to have attempted a coupling of mechanical capability, it is unknown if this is a "standard" capability of the respective code or if it is a one-off capability. For example there is a variant of TOUGH (TOUGH+/Hydrate) that has been coupled to the commercial geomechanics code, FLAC 3D (Itasca 2008), but it is not clear if all of the TOUGHREACT capability is available in this version. Similarly, the FEHM website (FEHM 2010) lists a "coupled stress module" as a capability, but it is not clear what behavior this module is capable of modeling on the mechanical side or if the capability is a general capability that could address the mechanical response that needs to be addressed for the Waste IPSC (e.g., hard rock/granite, clay/shale, and salt in the geosphere). The SIERRA suite of codes was designed to seamlessly permit multiple physics to be coupled and SIERRA's multiphysics coupling capability using SIERRA/FD to SIERRA/SM (Aria/Adagio) has recently been demonstrated (Stone et al. 2010; Hansen et al. 2010).

Large gaps also exist in modeling chemical processes and their couplings with other processes. First of all, there is no single code is able to fully account for all physical and chemical processes involved in the near field of a disposal system. For example, EQ3/6, which is widely used in nuclear waste management, is not able to simulate surface complexation, microbial reactions, radiolysis, and the partitioning of a chemical speciation between aqueous and gaseous phases (because it does not explicitly calculate the volume of gaseous phase). Second, the coupling of 
chemical processes with flow transport and mechanical deformation remains challenging. The resulting mathematical equations are generally nonlinear, involve sharp moving reaction fronts, and need to be solved over a large span of time scales, thus posing a serious issue for numerical solutions. Third, the data for extreme environments (e.g., for elevated temperature) that are needed for repository modeling are severely lacking. In addition, most of existing reactive transport codes were developed for non-radioactive contaminants, and they need to be adapted to account for radionuclide decay and ingrowth.

Because the problems of interest for the Waste IPSC are likely to result in relatively large computational models, a compact memory-usage footprint and a fast/robust solution procedure will be needed. This latter requirement points to the need for explicit solution methods that provide such a computational advantage over more traditional solution techniques (Arguello et al. 1998). Again, because of the relatively large computational models that are anticipated to be needed, a robust massively parallel processing (MPP) capability will also be required to provide reasonable turnaround times on the analyses that will be performed with the code. Finally, because the Waste IPSC applications will entail multiphysics, an effective and robust framework that will allow the efficient coupling of various physics to occur seamlessly is needed. Such a framework will need to provide an assortment of time-stepping and physics-coupling algorithms or strategies that permit physics of disparate time-scales and length-scales to be solved efficiently. Some of these frameworks and infrastructure needs are described in Section 5.

The capability of the codes described above to perform in a MPP environment is unclear, based on the available information. It appears that there is a variant of TOUGH2, called TOUGH2-MP (Zhang et al. 2008), that is intended for large-scale and high performance simulations running on distributed-memory or shared-memory parallel computers, including supercomputers, Linux clusters, workstations and multi-core personal computers. However, it is unclear if this capability is general and if there is an MPP version of TOUGHREACT. There is no explicit mention of a massively parallel capability in FEHM on the website (FEHM 2010) or in the User's Manual (Dash 2006), but the section on "Research Priorities" in Zyvoloski (2007), indicates that there may be an effort underway in this direction. As for SIERRA, the MPP capability was designed and developed from its inception into the code suite, so of the four simulators assessed, its MPP capability is without question and has been demonstrated on numerous applications (Stone et al. 2010; Hansen et al. 2010). 


\section{FRAMEWORKS AND INFRASTRUCTURE}

Verification, validation, and uncertainty quantification will be applied with varying levels of formality to modeling and simulation capabilities, as specified in the Waste IPSC V\&V Plan (Edwards et al. 2010). Implementation of these activities requires software frameworks and an enabling infrastructure as described in that $\mathrm{V} \& \mathrm{~V}$ plan.

\subsection{Enabling Infrastructure and Foundational Services}

The Enabling Infrastructure is required to support the practices and Quality Environment defined in the Waste IPSC V\&V Plan (Edwards et al. 2010). The Quality Environment is where developed and acquired code and data are imported for testing and assessment (Figure 5-1).

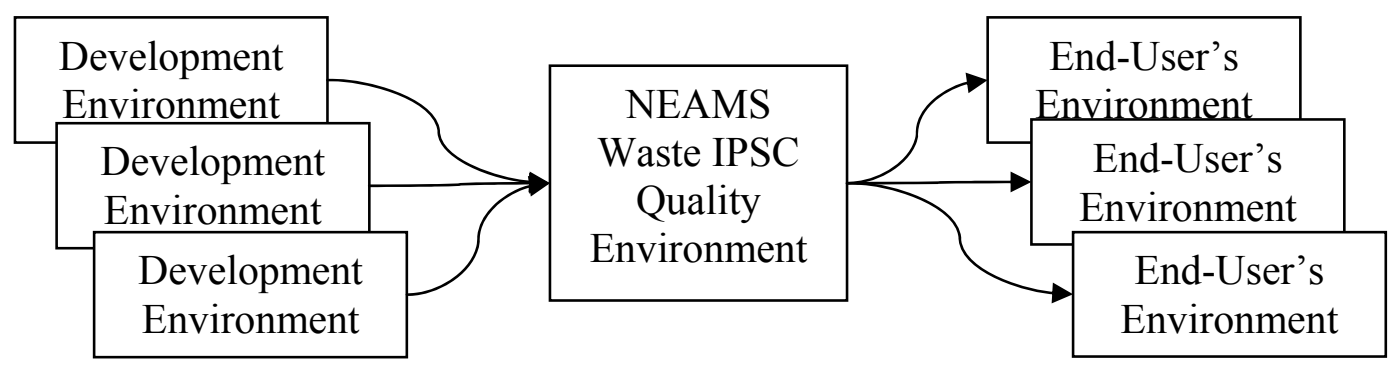

Figure 5-1. Flow of modeling and simulation capabilities from Development through Quality to End-User Environments

The Enabling Infrastructure is also required to support the challenge problem and milestones identified in Freeze et al. (2010). Many tools and capabilities of the Enabling Infrastructure will be needed for FY11 tasks and milestones. These tools and capabilities are identified in Table 5-1 in the areas of configuration management, requirements management, and project management. Additional details are provided in the following subsections. 
Table 5-1. Enabling Infrastructure - Tool Identification and Gap Analysis

\begin{tabular}{|c|c|c|c|c|}
\hline Area & Need & Priority & Selected Tool & Known Comparable Tools \\
\hline \multirow{6}{*}{$\begin{array}{l}\text { Configuration } \\
\text { Management }\end{array}$} & version control & high & Git & CVS, SVN \\
\hline & software build & high & CMake & make, pmake, imake, bjam \\
\hline & issue tracking & high & TBD & Trac, Bugzilla, Tracker \\
\hline & change request & low & & \\
\hline & test harness & high & TBD & CTest, CDash \\
\hline & software deployment & Low & & \\
\hline $\begin{array}{l}\text { Requirements } \\
\text { Management }\end{array}$ & $\begin{array}{l}\text { link requirements, } \\
\text { FEPs, tasks, codes, } \\
\text { tests }\end{array}$ & medium & TBD & $\begin{array}{l}\text { DOORS, ReqPro, TcSE, } \\
\text { Polarion, MS Excel Spreadsheet, } \\
\text { MS Access Database }\end{array}$ \\
\hline \multirow[t]{4}{*}{$\begin{array}{l}\text { Project } \\
\text { Management }\end{array}$} & team communication & High & TBD & $\begin{array}{l}\text { Wiki pages, Trac, Sharepoint, } \\
\text { TeamForge }\end{array}$ \\
\hline & $\begin{array}{l}\text { project-wide } \\
\text { communication }\end{array}$ & High & $\begin{array}{l}\text { project wide } \\
\text { decision }\end{array}$ & project-wide decision \\
\hline & $\begin{array}{l}\text { planning and } \\
\text { scheduling }\end{array}$ & High & TBD & Trac, MS Project, TeamForge \\
\hline & task management & High & TBD & $\begin{array}{l}\text { Trac, Sharepoint, Rally, } \\
\text { TeamForge }\end{array}$ \\
\hline
\end{tabular}

\subsubsection{Configuration Management}

The git and CMake (cross-platform make) tools have been selected for version control and software build. Both are open source, run on both Linux and Windows systems, and are in use successfully by other large code development projects at Sandia.

The test harness decision is not final at this time. The CTest tool and the associated CDash interface are delivered as part of CMake. This would be convenient. However, a full analysis of test needs, available tools, and ability to interface with git and CMake has not been conducted. This will be completed by December 2010 .

The issue tracking tool has not been selected. The desired functionalities of issue tracking in relationship to Project Management, Requirements Management and other Configuration Management areas have not been defined. A decision on an issue tracking tool will also be completed by December 2010 .

\subsubsection{Requirements Management}

There are many requirements management tools, ranging from simple to perhaps more complex than we need. The requirements management tool will manage requirements, FEPs, and the linkage of these to project management tasks, and to configuration-managed codes and tests. A design for the desired use of this tool is necessary before the tool is selected. 


\subsubsection{Project Management}

Decisions regarding program-wide and Waste IPSC inter-team communication are being considered. These tools and processes must be decided upon and implemented soon or the lack of program/team communication will impact productivity.

Other task management and planning/scheduling tools will probably be selected by their ability to interface easily with the communication tools. This decision will follow the project and team communication decisions.

Quality Environment Plans for FY11 (in anticipated order)

- Set up Quality Environment configurations like access and directory structure on the Quality Environment server.

- Install and test the project and team communication tools and any processes/services needed for productive communication.

- Install git and CMake and configure for Waste IPSC use.

- Test the import of codes and data into the Quality Environment, the version controlling of changes, and the build and run of codes.

\subsection{Analysis Workflow Framework}

From the analysis workflow perspective an end-user runs a sequence of codes to carry out a particular analysis or assessment activity. These codes typically include simulations, preprocessors for problem setup, postprocessors for analyzing results, and data manipulation utilities. An analysis workflow framework supports the planning, performing, tracking, and reproducing of a sequence of steps for an analysis activity. Each step potentially involves selecting and configuring a code to run, generating new inputs for the current step, selecting inputs from results generated by previous steps in the workflow, executing the code with these inputs, and archiving output results. Sufficient information must be maintained to track and reproduce the workflow step. In addition, workflow tracking information should document the end-users intent for each step in the workflow.

\subsubsection{Plan for Gap Analysis, Acquisition, and Development}

Management of the analysis workflow in computer-aided design and computer-aided engineering is a pervasive concern in industry and government laboratories. This domain of this concern has been identified as product lifecycle management, product data management, simulation data management, or analysis data management. Research and development in this domain is ongoing in multiple institutions, resulting in several potential commercial and public domain software frameworks. Each framework has inherent assumptions regarding (1) how the framework will be used and (2) needs for flexibility or extensibility.

The Waste IPSC plan for deployment of an analysis workflow framework assumes that there exists one or more suitable commercial, government laboratory, or public domain solutions for an analysis workflow framework. The acquisition plan is as follows: 
1. Seek collaborative programmatic partners to increase the likelihood of arriving at an analysis workflow framework that is shared by multiple DOE programs and laboratories.

2. Survey existing studies in this domain to understand and articulate the major components, needs, and goals of analysis workflow frameworks and their major components.

3. Identify the corresponding major components, needs, and goals that are required for the Waste IPSC.

4. Survey existing commercial, government laboratory, and public domain solutions - the analysis workflow frameworks or framework components. Leverage existing analogous surveys. Perform this survey with programmatic partners.

5. Evaluate the suitability of existing solutions. Perform this evaluation with programmatic partners.

6. Acquire an initial (prototype) analysis workflow framework, or acquire and integrate framework components. This acquisition assumes that a suitable framework or framework components are identified.

7. Apply the initial (prototype) framework to carry out the Waste IPSC challenge problem milestones and V\&V and UQ assessments.

8. Evaluate the application of the prototype framework to determine whether (a) the initial solution should is sufficient or (b) a new acquisition or development activity is warranted.

\subsubsection{Potential Collaborators}

\section{DOE-NE Used Fuel Disposition (UFD) Campaign}

The UFD Campaign is exploring the deployment of an analysis workflow framework to support performance assessment for a range of nuclear waste disposal scenarios. This framework is intended to support modeling and simulation capabilities at the PA scale, analogous to those modeling and simulation capabilities used in the Yucca Mountain Project (YMP). The UFD campaign is currently utilizing the commercial product GoldSim, which was also used by YMP.

The Waste IPSC established a dialogue with the UFD Campaign to share information and explore the potential for collaboration.

\section{DOE Advanced Simulation Capability for Environmental Management (ASCEM)}

The ASCEM program plan includes deployment of a framework for uncertainty quantification and data management. This framework is intended to support modeling and simulation capabilities at the continuum scale with early priorities for transport phenomena in the far field and later priorities for coupled phenomena in the near field.

The Waste IPSC has initiated a dialogue with ASCEM to explore potential collaboration for both frameworks and modeling and simulation capabilities.

\section{DOE Advanced Simulation and Computing (ASC)}

In November 2009 a study was commissioned by ASC at SNL to develop a set of analysis data management requirements and assess the ASC integrated DART Workbench against these requirements. Several commercial products were also assessed against these requirements. The 
results of this study were presented in June 2010. One result is a recommendation to perform a pilot project to acquire, exercise, and evaluate a commercial product.

The Waste IPSC established a partnership with ASC at SNL with the objective of collaborating on this pilot project.

\subsubsection{Framework Components Needs and Goals}

Most of the frameworks needs and goals identified in this section are derived from and traceable to the sources identified in the following table. The "label for identification" is used in subsequent tables in this section to reference these sources.

\begin{tabular}{|l|l|}
\hline Source & Label for identification \\
\hline $\begin{array}{l}\text { NEAMS Waste Forms and Systems Integrated Performance and } \\
\text { Safety Codes System Design Specification (SNL 2009) }\end{array}$ & NEAMS:Waste:SDS \\
\hline $\begin{array}{l}\text { NEAMS Waste Integrated Performance and Safety Codes } \\
\text { Verification and Validation Plan (Edwards et al. 2010) }\end{array}$ & NEAMS:Waste:V\&V \\
\hline SNL Framework Assessment Tool (Vaughn 2010) & SNL:Vaughn \\
\hline $\begin{array}{l}\text { ASC SNL Analysis Data Management (ADM) Study: } \\
\text { Requirements and Recommendations Summary (Baca 2010) }\end{array}$ & ASC:SNL:ADM \\
\hline
\end{tabular}

The long-term viability of the Waste IPSC requires confidence in the sustainability of the selected analysis workflow framework components. Sustainability goals are listed in the following table.

\begin{tabular}{|l|l|}
\hline Functional Goals & Sources \\
\hline Support end-users at multiple independent sites. & $\begin{array}{l}\text { NEAMS:Waste:SDS } \\
\text { NEAMS:Waste:V\&V }\end{array}$ \\
\hline Support access from distributed sites. & NEAMS:Waste:SDS \\
\hline Support multiple distributed work groups. & ASC:SNL:ADM \\
\hline Support corporate enterprise / Sandia infrastructure. & ASC:SNL:ADM \\
\hline Non-Functional Goals & Sources \\
\hline $\begin{array}{l}\text { Cost of acquisition and sustainment is affordable for the Waste } \\
\text { IPSC program element and its end-users. }\end{array}$ & ASC:SNL:ADM \\
\hline Cost of migration is affordable. & ASC:SNL:ADM \\
\hline $\begin{array}{l}\text { Confidence in supplier's future viability and responsiveness for } \\
\text { support, maintenance, and enhancement. }\end{array}$ & ASC:SNL:ADM \\
\hline
\end{tabular}

The major analysis workflow framework components of the Waste IPSC are identified with a brief statement of need for each framework component. Subsequent tables list an initial set of goals for each identified framework component. This initial set of needs and goals does not represent a comprehensive requirements analysis for an analysis workflow framework. 
Analysis Workflow User Interface: Interact with the user to define and executed the analysis workflow including (but not limited to) selection of codes and input files, generation or modification of input files, selection of execution servers, and specification of data management formality.

Analysis Data Management: Manage analysis data including (but not limited to) problem description documents, representations or realizations of the physical system (e.g., meshed geometric representations), input files of parameters for numerical models (e.g., material properties, boundary conditions), numerical solution algorithm parameters, and output simulation results. Analysis data management includes configuration management of files, descriptive meta-data, and provenance meta-data.

Model Parameter Management: Manage model parameters such as material properties and chemistry parameters. Model parameter management includes configuration management of individual parameters, descriptive meta-data, and provenance meta-data.

Code Execution Management: Manage execution of codes in the analysis workflow on the end-user's local workstation and remote servers. Execution management includes identification of all code inputs, movement of code inputs to the remote server as necessary, execution of the code, and movement of code outputs from the remote server as necessary.

Iterative Analysis Tools: Support an extensible collection of tools that iterate an analysis workflow with varying parameters; typically for the purpose of uncertainty quantification, sensitivity analysis, or optimization.

Analysis Setup Tools: Support an extensible collection of analysis setup tools including (but not limited to) geometry definition and meshing, coupled code assembly, input file generation, uncertainty quantification sampling iterations.

Post Processing Tools: Support an extensible collection of analysis results post processing tools including (but not limited to) plotting, visualization, animation, and statistical reductions.

Data Exchange / Translation Tools: Support an extensible collection of tools to translate analysis data files from the output format of one simulation or tool to the input format of another simulation or tool.

Simulation Codes: Support the extensible collection of codes that implement the Waste IPSC's integrated modeling and simulation capabilities. 


\section{Analysis Workflow User/Client Interface}

\begin{tabular}{|l|l|}
\hline Functional Goals & Sources \\
\hline $\begin{array}{l}\text { Assist the user in the design, creation, reuse, and update of } \\
\text { workflows. }\end{array}$ & $\begin{array}{l}\text { SNL:Vaughn } \\
\text { ASC:SNL:ADM }\end{array}$ \\
\hline $\begin{array}{l}\text { Support system modeling by visually integrating subsystem models } \\
\text { and provide traceability among integrated subsystems. }\end{array}$ & $\begin{array}{l}\text { SNL:Vaughn } \\
\text { ASC:SNL:ADM }\end{array}$ \\
\hline $\begin{array}{l}\text { Provide a method for generating environments, model inputs, and } \\
\text { boundary conditions for the workflow without manual scripting/ } \\
\text { assistance. }\end{array}$ & SNL:Vaughn \\
\hline $\begin{array}{l}\text { Support workflow management including executing and restarting } \\
\text { workflow tools/codes. }\end{array}$ & $\begin{array}{l}\text { SNL:Vaughn } \\
\text { ASC:SNL:ADM }\end{array}$ \\
\hline $\begin{array}{l}\text { Provide an integration and regression test capability for workflows } \\
\text { that can be used prior to execution. }\end{array}$ & $\begin{array}{l}\text { NEAMS:Waste:SDS } \\
\text { NEAMS:Waste:V\&V }\end{array}$ \\
\hline $\begin{array}{l}\text { Fully integrated to SNL security infrastructure and able to support } \\
\text { different levels of rigor. }\end{array}$ & ASC:SNL:ADM \\
\hline Nervices must be accessible and operable from other DOE sites. & NEAMS:Waste:SDS \\
\hline Run in a Microsoft Windows environment. & SNL:Vaughn \\
\hline
\end{tabular}




\section{Analysis Data Management}

\begin{tabular}{|c|c|}
\hline Functional Goals & Sources \\
\hline $\begin{array}{l}\text { Retention and retrieval of input and output data associated with } \\
\text { specific analyses needed to reproduce computations. }\end{array}$ & NEAMS:Waste:SDS \\
\hline $\begin{array}{l}\text { Support defined and extensible provenance / traceability data and } \\
\text { meta-data associated with the analysis data. Traceability can } \\
\text { include references to externally configuration managed data. }\end{array}$ & $\begin{array}{l}\text { NEAMS:Waste:SDS } \\
\text { SNL:Vaughn } \\
\text { ASC:SNL:ADM }\end{array}$ \\
\hline Provide long-term storage of results, inputs, and intermediate steps & $\begin{array}{l}\text { ASC:SNL:ADM } \\
\text { NEAMS:Waste:SDS }\end{array}$ \\
\hline $\begin{array}{l}\text { Support configuration management of data and meta-data. } \\
\text { Configuration management includes support for data retention } \\
\text { policies. }\end{array}$ & $\begin{array}{l}\text { NEAMS:Waste:SDS } \\
\text { SNL:Vaughn } \\
\text { ASC:SNL:ADM }\end{array}$ \\
\hline Support a definable analysis data management lifecycle. & ASC:SNL:ADM \\
\hline Support accessible, searchable, and navigable meta-data and data. & $\begin{array}{l}\text { NEAMS:Waste:SDS } \\
\text { SNL:Vaughn } \\
\text { ASC:SNL:ADM }\end{array}$ \\
\hline $\begin{array}{l}\text { Manage traceability to data that is distributed among computational } \\
\text { resources. }\end{array}$ & ASC:SNL:ADM \\
\hline $\begin{array}{l}\text { Support security policies in regard to utilization of resources and } \\
\text { accessibility to data. }\end{array}$ & $\begin{array}{l}\text { NEAMS:Waste:SDS } \\
\text { ASC:SNL:ADM }\end{array}$ \\
\hline $\begin{array}{l}\text { Handle large volumes of data; measured by both the number of } \\
\text { files and the size of files. }\end{array}$ & $\begin{array}{l}\text { NEAMS:Waste:SDS } \\
\text { ASC:SNL:ADM }\end{array}$ \\
\hline Manage analysis data files of varied and evolving formats & ASC:SNL:ADM \\
\hline Report generation: including results, resources used, activity logs & NEAMS:Waste:SDS \\
\hline $\begin{array}{l}\text { Fully integrated to SNL security infrastructure and able to support } \\
\text { different levels of rigor. }\end{array}$ & $\begin{array}{l}\text { ASC:SNL:ADM } \\
\text { NEAMS:Waste:SDS }\end{array}$ \\
\hline Services must be accessible and operable from other DOE sites & NEAMS:Waste:SDS \\
\hline
\end{tabular}




\section{Model Parameter Management}

\begin{tabular}{|l|l|}
\hline Functional Goals & Sources \\
\hline $\begin{array}{l}\text { Support configuration management of model parameters such as } \\
\text { material properties. Management of parameters will be at a finer } \\
\text { granularity as compared to analysis data files. }\end{array}$ & NEAMS:Waste:SDS \\
\hline $\begin{array}{l}\text { Support identification of models and versions of models using } \\
\text { particular parameters or cohesive collections of parameters. }\end{array}$ & $\begin{array}{l}\text { NEAMS:Waste:SDS } \\
\text { ASC:SNL:ADM }\end{array}$ \\
\hline Support association of uncertainties with model parameters. & NEAMS:Waste:SDS \\
\hline $\begin{array}{l}\text { Support traceability to source of parameters and uncertainties } \\
\text { such as credible references, experimental results, and sub- } \\
\text { continuum-scale analyses. }\end{array}$ & NEAMS:Waste:SDS \\
\hline $\begin{array}{l}\text { Provide configuration management of the properties, tools, and } \\
\text { codes and historic log of changes. }\end{array}$ & $\begin{array}{l}\text { SNL:Vaughn } \\
\text { ASC:SNL:ADM } \\
\text { NEAMS:Waste:SDS } \\
\text { NEAMS:Waste:V \&V }\end{array}$ \\
\hline $\begin{array}{l}\text { Provide an extensible provenance / traceability / linkage of the } \\
\text { properties, tools, and codes. }\end{array}$ & $\begin{array}{l}\text { SNL:Vaughn } \\
\text { ASC:SNL:ADM } \\
\text { NEAMS:Waste:SDS }\end{array}$ \\
\hline $\begin{array}{l}\text { Allow the user to search and navigate properties, tools, and codes } \\
\text { Fully integrated to SNL security infrastructure and able to support } \\
\text { different levels of rigor }\end{array}$ & $\begin{array}{l}\text { NEAS:Waste:SDS } \\
\text { ASC:SNL:ADM }\end{array}$ \\
\hline Services must be accessible and operable from other DOE sites & NEAMS:Waste:SDS \\
\hline
\end{tabular}




\section{Code Execution Management}

\begin{tabular}{|c|c|}
\hline Functional Goals & Sources \\
\hline $\begin{array}{l}\text { Provide and submit an integrated workflow of codes to local and } \\
\text { remote Linux and Windows execution environments including high } \\
\text { performance computing (HPC) clusters. }\end{array}$ & $\begin{array}{l}\text { ASC:SNL:ADM } \\
\text { SNL:Vaughn } \\
\text { ASC:SNL:ADM }\end{array}$ \\
\hline $\begin{array}{l}\text { Provide and submit a portion of an integrated workflow of codes } \\
\text { that inputs intermediate data saved from a previously executed } \\
\text { workflow. }\end{array}$ & SNL:Vaughn \\
\hline $\begin{array}{l}\text { Provide for the reuse of previous results in new or modified } \\
\text { workflows }\end{array}$ & $\begin{array}{l}\text { SNL:Vaughn } \\
\text { ASC:SNL:ADM }\end{array}$ \\
\hline $\begin{array}{l}\text { Provide for traceable movement of data between workflow } \\
\text { tools/codes and between execution environments. }\end{array}$ & $\begin{array}{l}\text { NEAMS:Waste:SDS } \\
\text { SNL:Vaughn } \\
\text { ASC:SNL:ADM }\end{array}$ \\
\hline $\begin{array}{l}\text { Provide for movement of data into and out of analysis data } \\
\text { management. }\end{array}$ & $\begin{array}{l}\text { NEAMS:Waste:SDS } \\
\text { SNL:Vaughn }\end{array}$ \\
\hline $\begin{array}{l}\text { Support code execution with specified versions of input data } \\
\text { obtained from analysis data management. }\end{array}$ & NEAMS:Waste:SDS \\
\hline $\begin{array}{l}\text { Track temporary/intermediate workflow data to support review and } \\
\text { verification of that data even when that data is not explicitly } \\
\text { configuration managed. }\end{array}$ & NEAMS:Waste:SDS \\
\hline $\begin{array}{l}\text { Support security policies in regard to utilization of resources and } \\
\text { accessibility to data. }\end{array}$ & NEAMS:Waste:SDS \\
\hline $\begin{array}{l}\text { Track code execution history including codes executed, execution } \\
\text { environment, data input, data output, and resources used. } \\
\text { Traceability must be bidirectional. }\end{array}$ & $\begin{array}{l}\text { NEAMS:Waste:SDS } \\
\text { SNL:Vaughn }\end{array}$ \\
\hline $\begin{array}{l}\text { Provide an open and extensible interface for integrating tools and } \\
\text { simulation codes from a variety of sources and a variety of } \\
\text { sciences. }\end{array}$ & $\begin{array}{l}\text { SNL:Vaughn } \\
\text { NEAMS:Waste:V\&V } \\
\text { ASC:SNL:ADM }\end{array}$ \\
\hline $\begin{array}{l}\text { Support diverse analysis tools and a standardized and extensible } \\
\text { interface for the inclusion of new analysis tools. }\end{array}$ & $\begin{array}{l}\text { NEAMS:Waste:V\&V } \\
\text { SNL:Vaughn }\end{array}$ \\
\hline $\begin{array}{l}\text { Allow the inclusion of codes and data from a variety of sources and } \\
\text { a variety of sciences to interface in workflows. }\end{array}$ & $\begin{array}{l}\text { NEAMS:Waste:V\&V } \\
\text { ASC:SNL:ADM }\end{array}$ \\
\hline $\begin{array}{l}\text { Support background execution and data movement so that control } \\
\text { is returned to the user prior to completion. Provide status on } \\
\text { workflow progress including reported warnings/errors. }\end{array}$ & ASC:SNL:ADM \\
\hline $\begin{array}{l}\text { Support execution of tools and simulations on local Linux-based } \\
\text { workstations, remote Linux-based servers, and remote HPC clusters } \\
\text { with queuing system and local file systems. }\end{array}$ & ASC:SNL:ADM \\
\hline $\begin{array}{l}\text { Fully integrated to SNL security infrastructure and able to support } \\
\text { different levels of rigor. }\end{array}$ & $\begin{array}{l}\text { ASC:SNL:ADM } \\
\text { NEAMS:Waste:SDS }\end{array}$ \\
\hline Services must be accessible and operable from other DOE sites & NEAMS:Waste:SDS \\
\hline
\end{tabular}




\section{Iterative Analysis Tools}

\begin{tabular}{|l|l|}
\hline Functional Goals & Sources \\
\hline $\begin{array}{l}\text { Support iterative execution in support of sampling-based } \\
\text { uncertainty quantification and optimization workflows. }\end{array}$ & SNL:Vaughn \\
\hline $\begin{array}{l}\text { Support tools to perform sensitivity analysis and uncertainty } \\
\text { analysis. }\end{array}$ & SNL:Vaughn \\
\hline Ability to perform sensitivity analysis and uncertainty analysis & SNL:Vaughn \\
\hline $\begin{array}{l}\text { Support at least LHS MC probabilistic analyses where the end-user } \\
\text { has control over sampling attributes. }\end{array}$ & SNL:Vaughn \\
\hline Support analyses of both epistemic and aleatory uncertainty. & SNL:Vaughn \\
\hline
\end{tabular}

\section{Analysis Setup Tools}

\begin{tabular}{|l|l|}
\hline Functional Goals & Sources \\
\hline $\begin{array}{l}\text { Support an extensible collection of analysis setup tools and a } \\
\text { standardized interface for the inclusion of new tools. }\end{array}$ & NEAMS:Waste:V\&V \\
\hline $\begin{array}{l}\text { Support tools for pre-processing tools including input file } \\
\text { generation. }\end{array}$ & ASC:SNL:ADM \\
\hline $\begin{array}{l}\text { Support tools for geometry definition and meshing (and other } \\
\text { scientific analysis) }\end{array}$ & NEAMS:Waste:V\&V \\
\hline Support tools for coupled code assembly. & \\
\hline
\end{tabular}

\section{Post Processing Tools}

\begin{tabular}{|l|l|}
\hline Functional Goals & Sources \\
\hline $\begin{array}{l}\text { Support an extensible collection of post-processing tools and a } \\
\text { standardized interface for the inclusion of new tools. }\end{array}$ & $\begin{array}{l}\text { NEAMS:Waste:V\&V } \\
\text { ASC:SNL:ADM }\end{array}$ \\
\hline $\begin{array}{l}\text { Support post processing tools including plotting, visualization, } \\
\text { animation, and statistical reductions. }\end{array}$ & \\
\hline
\end{tabular}

\section{Data Exchange / Translation Tools}

\begin{tabular}{|l|l|}
\hline Functional Goals & Sources \\
\hline $\begin{array}{l}\text { Translate analysis data files from the output format of one } \\
\text { simulation or tool to the input format of another simulation or tool. }\end{array}$ & ASC:SNL:ADM \\
\hline
\end{tabular}

\section{Simulation Codes}

\begin{tabular}{|l|l|}
\hline Functional Goals & Sources \\
\hline $\begin{array}{l}\text { Support an extensible collection of simulation codes and a } \\
\text { standardized interface for the inclusion of new codes. }\end{array}$ & NEAMS:Waste:V\&V \\
\hline $\begin{array}{l}\text { Support the collection of codes that implement the Waste IPSC } \\
\text { integrated modeling and simulation capabilities. }\end{array}$ & NEAMS:Waste:V\&V \\
\hline
\end{tabular}




\subsubsection{Survey of Existing Solutions}

An initial survey of existing analysis workflow frameworks and framework components identified the public domain, commercial domain, and U.S. government laboratories is listed here. This initial list is not exhaustive and other potential solutions may be identified through dialogue with the potential collaborators (Section 5.2.2). The descriptions given are summaries of information provided by the companies or organizations and are not objective evaluation of the tools. Each of these solutions addresses some of the identified needs and goals. A systematic evaluation is in progress.

\section{Public Domain}

- SALOME: Open CASCADE and consortium of partners

SALOME is a Linux platform for pre- and post-processing and integration of external third-party numerical codes for geometry, mesh and solver computations. http://www.salome-platform.org

- Kepler: Kepler Project Team (core team are from branches of University of California)

Kepler is a java-based application that is maintained for Windows, OSX, and Linux and helps scientific users share and reuse data, workflows, and components to address common needs. https://kepler-project.org

- OpenTURNS: partnership of three French/European companies

OpenTURNS is Unix/Linux software to perform probabilistic studies including quantifying uncertainty, propagating and ranking uncertainty, and sensitivity analysis. http://trac.openturns.org

\section{Commercial}

- GoldSim: GoldSim Technology Group

GoldSim is a hybrid of several simulation approaches, combining a Monte Carlo simulator, system dynamics, and discrete event simulation. http://www.goldsim.com

- PHX AnalysisLibrary: Phoenix Integration, Inc.

AnalysisLibrary is a managed shared file system that organizes and stores engineering data for search and reuse and that builds on tools and techniques used by the team. http://www.phoenix-int.com/software/data management.php

- PHX ModelCenter: Phoenix Integration, Inc.

PHX ModelCenter graphically integrates analysis programs, passes design parameters from one program to another, integrates with simulation and optimization plug-ins, and contains a tool for incorporating or wrapping other algorithms/applications. http://www.phoenix-int.com/software/phx_modelcenter.php 
- Windchill: Parametric Technology Corporation (PTC)

Windchill is PTC's Product Lifecycle Management software for managing product content and processes. http://www.ptc.com/products/windchill/

- TeamCenter: Siemens

TeamCenter provides a family of tools that includes a development environment for scientific software, a simulation environment, workflow capabilities, and system/requirements management.

http://www.plm.automation.siemens.com/en_us/products/teamcenter

- SimManager: MSC Software

SimManager is a build and test management system that manages and automates simulations and workflows and supports third party applications and tools. http://www.mscsoftware.com/Products/Virtual-Build-And-TestManagement/SimManager.aspx

- HyperWorks Enterprise: Altair Engineering

HyperWorks provides an open-architecture solution including modeling, analysis, visualization and data management solutions for linear, nonlinear, structural optimization, fluid-structure interaction, and multi-body dynamics applications.

http://www.altairhyperworks.com

- Workbench and EKM: ANSYS

Workbench ties together the multiphysics analysis simulation process, automates parameter management, and integrates optimization tools. Workbench connects to Engineering Knowledge Manager (EKM) to provide a centralized repository for data and process storage and management. http://www.ansys.com/products/ekm

- Abaqus: SIMULIA/Dassault Systemes

(there is also an independent CATIA Community for Dassault Systemes tools)

Abaqus is a family of tools that includes multiphysics solutions and simulation, data, and process management. http://www.3ds.com/products/simulia/overview

- Simulation Data Management Framework: Engineous Software, Inc.

Engineous Software was acquired by Dassault Systemes in 2008 with the intention of improving Abaqus. 
- OpenPDM: PROSTEP, Inc.

OpenPDM is a cross-system product data management (PDM) and integration tool for improving the coordination of distributed development processes and lifecycle management. http://www.prostep.com/our-products/openpdm/?L=1

- OPTIMUS: Noesis Solutions

Noesis Solutions was acquired by Tokyo-based Cybernet Systems in July 2010. Optimus automates simulation tasks across multiple engineering disciplines, assists with optimization and Design of Experiments (DOE), and links to legacy and third party codes and models.

http://www.noesissolutions.com/index.php?col=/products\&doc=optimus

\section{$\underline{\text { US Government Laboratory }}$}

- DART Workbench : Sandia National Laboratories

A collection of integrated software tools to help analysts reduce the time required to build, analyze, and understand complex finite element analysis simulations. It provides convenient resource links, file management, metadata editor, tools for teaming, the ability to add engineering notes to objects, and model management capabilities. https://dta.ran.sandia.gov/dart/ 


\section{SUMMARY}

This year-end progress report documents the FY10 status of the acquisition, development, and integration of code capabilities supporting the NEAMS Waste IPSC. Activities in FY10 focused on performing initial gap analyses to identify candidate codes and tools to support (1) multifidelity THCM modeling and simulation capabilities, and (2) frameworks and enabling infrastructure. The current Waste IPSC strategy is to acquire and integrate the necessary Waste IPSC capabilities wherever feasible, and develop only those capabilities that cannot be acquired or suitably integrated, verified, or validated.

The Waste IPSC gap analysis is a continuing process and the analysis documented in this report serves only as a starting point for a full analysis planned to be conducted in future years. Further development and integration of THCM code capabilities and frameworks and enabling infrastructure will be documented in future reports over the multi-year duration of the project. 


\section{REFERENCES}

Abramczyk, H., B. Brozek-Pluska, K. Kurczewski, I. Szymczyk, T. Blaszczyk, H. Scholl and W. Czajkowski 2006. Femtosecond transient absorption, Raman, and electrochemistry studies of tetrasulfonated copper phthalocyanine in water solutions. Journal of Physical Chemistry A 110, $8627-8636$.

Advocet, T., P. Jollivet, J.L. Crovisier, and M. del Nero 2001. Long-term alteration mechanisms in water for SON68 radioactive borosilicate glass. Journal of Nuclear Materials, 298, 55-62.

Aertsens, M. 1999. Testing the Grambow glass dissolution model by comparing it with Monte Carlo simulation results. Materials Research Society Symposium Proceedings 556, 409-419.

Aertsens, M., and D. Ghaleb 2001. New techniques for modelling glass dissolution, Journal of Nuclear Materials, 298, 37-46.

Agence Nationale pour la Gestion des Déchets Radioactifs (ANDRA) 2005. Dossier 2005: Argile. Tome: Safety Evaluation of a Geological Repository (English translation: original documentation written in French remains ultimately the reference documentation). http://www.andra.fr/international/pages/en/menu21/waste-management/research-anddevelopment/dossier-2005-1636.html

Allison, J.D., D.S. Brown, and K.J. Novo-Gradac 1990. MINTEQA2/PRODEFA2-A geochemical assessment model for environmental systems-version 3.0 user's manual. Environmental Research Laboratory, Office of Research and Development, U.S. Environmental Protection Agency, Athens, Georgia, 106 p.

Alonso, E.E., A. Gens, and A. Josa 1990. A constitutive model for partially saturated soils, Géotechnique, 40(3), pp. 405-430.

ANSYS, 2010. ANSYS, Inc. website - http://www.ansys.com (last accessed September 20, 2010).

Appelo, C.A.J., and D. Postma 1999. A consistent model for surface complexation on birnessite ( $-\mathrm{MnO} 2$ ) and its application to a column experiment, Geochimica et Cosmochimica Acta, (63), 3039-3048.

Appelo, C.A.J., and P. Wersin 2007. Multicomponent diffusion modeling in clay systems with application to the diffusion of tritium, iodide and sodium in Opalinus Clay, Env. Sci. Technol., $41,5002-5007$.

Appelo, C.A.J., L.R. Van Loon, and P. Wersin 2010. Multicomponent diffusion of a suite of tracers ( $\mathrm{HTO}, \mathrm{Cl}, \mathrm{Br}, \mathrm{I}, \mathrm{Na}, \mathrm{Sr}, \mathrm{Cs}$ ) in a single sample of Opalinus Clay, Geochimica et Cosmochimica Acta 74, 1201-1219. 
Argüello, J. G., C.M. Stone, and A.F. Fossum 1998. Progress on the development of a threedimensional capability for simulating large-scale complex geologic processes," Int. J. Rock Mech. Min. Sci., Vol. 35 4/5:469-470, Paper No. 083.

Argyris, D., N.R. Tummala, and A. Striolo 2008. Molecular structure and dynamics in thin water films at the silica and graphite surfaces, Journal of Physical Chemistry C, 112, 1358713599.

Argyris, D., D.R. Cole, and A. Striolo 2009. Hydration struture on crystalline silica substrates, Langmuir, 25, 8025-8035.

Aubertin, M., D.E. Gill, and B. Ladanyi 1991. A unified viscoplastic model for the inelastic flow of alkali halides, Mech. of Mat., 11, 63-82.

Baca, T. 2010. Analysis Data Management (ADM) Study: Requirements and Recommendations Summary. Sandia National Laboratories Official Use Only (OUO) Briefing to the Comp/Sim Leadership Team, Albuquerque, New Mexico, June 15, 2010.

Ball, J.W. and D.K. Nordstrom 1991. WATEQ4F-User's manual with revised thermodynamic data base and test cases for calculating speciation of major, trace and redox elements in natural waters. U.S. Geological Survey Open-File Report 90-129.

Bandstra, J.Z., and S.L. Brantley 2008. Surface evolution of dissolving minerals investigated with a kinetic Ising model, Geochimica et Cosmochimica Acta, 72, 2587-2600.

Bennett, M., and S. Rohani 2001. Solution of population balance equations with a new combined Lax-Wendroff/Crank-Nicholson method. Chem. Eng. Sci. 56, 6623-6633.

Bethke, C.M. 1996. Geochemical Reaction Modeling, Concepts and Applications. Oxford University Press, New York.

Bethke, C.M. 1998. The Geochemist's Workbench (Release 3.0): A User's Guide to Rxn, Act2, Tact, React, and Gtplot. Hydrology Program, University of Illinois. 184 p.

Blanford, M.L., M.W. Heinstein, and S.W. Key 2001. JAS3D - A Multi-Strategy Iterative Code for Solid Mechanics Analysis, Users' Instructions, Release 2.0, Draft SAND Report, Sandia National Laboratories, Albuquerque, New Mexico.

Brannon, R.M., A.F. Fossum, and O.E. Strack 2009. Kayenta: Theory and User's Guide, SAND2009-2282, Sandia National Laboratories, Albuquerque, New Mexico.

Brantley, S.L., and L. Stillings 1996. Feldspar dissolution at $25^{\circ} \mathrm{C}$ and low $\mathrm{pH}$, American Journal of Science, 296, 101-127.

Brantley, S.L., and L.L. Stillings 1997. Reply to comment: Feldspar dissolution at $25^{\circ} \mathrm{C}$ and low pH, American Journal of Science, 297, 1021-1032. 
Brantley, S.L. 2004. Reaction kinetics of primary rock-forming minerals under ambient conditions, Surface and Ground Water, Weathering, and Soils, edited by J. I. Drever, pp. 73-117, Elsevier.

Brantley, S.L. 2008. Kinetics of mineral dissolution, Kinetics of Water-Rock Interaction edited by S.L. Brantley, J.D. Kubicki, and A.F. White, pp. 151-210, Springer-Science.

Bryan, C.R., K.B. Helean, B.D. Marshall, and P.V. Brady 2009. Feldspar dissolution rates in the Topopah Spring Tuff, Yucca Mountain, Nevada, Applied Geochemistry, 24(11), 2133-2143.

Cabaret, D., M. Le Grand, A. Ramos, A.-M. Flank, S. Rossano, L. Galoisy, G. Calas, and D. Ghaleb 2001. Medium range structure of borosilicate glasses from Si K-edge XANES: a combined approach based on multiple scattering and molecular dynamics calculations, Journal of Non-Crystalline Solids, 289, 1-8.

Cailleteau C., F. Angeli, F. Devreux, S. Gin, J. Jestin, P. Jollivet, and O. Spalla 2008. Insight into silicate-glass corrosion mechanisms, Nature Materials, 7, 978-983.

Carey, G.R., P.J.V. Geel, and J.R. Murphy 1999. BioRedox-MT3DMS V2.0: A Coupled Biodegradation-Redox Model for Simulating Natural and Enhanced Bioremediation of Organic Pollutants - User's Guide. Technical report, Conestoga-Rovers \& Associates, Waterloo, Ontario, Canada.

Casey, W.H., A.C. Lasaga, and G.V. Gibbs 1990. Mechanisms of silica dissolution as inferred from the kinetic isotope effect, Geochimica et Cosmochimica Acta, 54, 3369-3378.

Casey, W. H., and C. Ludwig 1996. The mechanism of dissolution of oxide minerals, Nature, 381, 506-509.

Chan, K.S., D.E. Munson, and S.R. Bodner 1999. Creep deformation and fracture in rock salt, in Fracture of Rock, ed. M. H. Aliabadi, WIT Press, Boston, MA.

Cormack, A.N., J. Du, and T.R. Zeitler 2002. Alkali ion migration mechanisms in silicate glasses probed by molecular dynamics simulations, Physical Chemistry Chemical Physics, 4, 3193-3197.

Crawford, J. 1999. Geochemical modeling - A review of current capabilities and future directions. SNV Report 262, Naturvardsverket, Swedish Environmental Protection Agency.

Criscenti, L.J., J.D. Kubicki, and S.L. Brantley 2006. Silicate glass and mineral dissolution: Calculated reaction paths and activation energies for hydrolysis of a $\mathrm{Q}^{3} \mathrm{Si}$ by $\mathrm{H}_{3} \mathrm{O}^{+}$using ab initio methods, Journal of Physical Chemistry A, 110, 198-206. 
Criscenti, L.J., S.L. Brantley, K.T. Mueller, N. Tsomaia, and J.D. Kubicki 2005. Theoretical and ${ }^{27}$ Al CPMAS NMR investigation of aluminum coordination changes during aluminosilicate dissolution, Geochimica et Cosmochimica Acta, 69, 2205-2220.

Cruz-Chu, E.R., A. Aksimentiev, and K. Schulten 2006. Water-silica force field for simulating nanodevices, Journal of Physical Chemistry B, 110, 21497-21508.

Dash, Z.V. 2006. Software Users Manual (UM) for the FEHM Application Version 2.30, 100862.30-00, Earth and Environmental Sciences Division, Los Alamos National Laboratory, Los Alamos, New Mexico.

Davis, J.A., and D.B. Kent 1990. Surface complexation modeling in aqueous geochemistry, in Mineral-Water Interface Geochemistry, Reviews in Mineralogy Vol. 23, M.F. Hochella and A.F. White, eds., Mineralogical Society of America, Chap. 5, 177-260.

Dewers, T., and P. Ortoleva 1990. A coupled reaction/transport/mechanical model for intergranular pressure solution, stylolites, and differential compaction and cementation in clean sandstones, Geochimica et Cosmochimica Acta 54, 1609-1625.

Dewers, T. and P. Ortoleva 1992b. Formation of stylolites, marl/limestone alterations, and clay seams through unstable chemical compaction of argillaceous carbonates, in Diagenesis IV, K.H. Wolf and G.V. Chilingarian, eds., Chapter 7, Elsevier. pp. 155-216.

Dewers, T. and Z. Reches 2004. Alteration of fresh fault gouge from focal depths of recent earthquakes in deep mines, EOS Trans. AGU 85 (47) Fall Meet. Suppl., Abstract S41B-0969.

Dooling, D.J. and L.J. Broadbelt 2001. Generic Monte Carlo tool for kinetic modeling, Industrial and Engineering Chemistry Research, 40, 522-529.

Dove, P. M. 1994. The dissolution kinetics of quartz in sodium chloride solutions at $25^{\circ}$ to $300^{\circ} \mathrm{C}$, American Journal of Science, 291, 665-712.

Dove, P.M. 1995. Kinetic and thermodynamic controls on silica reactivity in weathering environments, in Chemical Weathering Rates of Silicate Melts, Mineralogical Society of America, Short Course Vol. 31, A.F. White and S.L. Brantley, eds., pp. 236-290.

Dove, P.M., and S.F. Elston 1992. Dissolution kinetics of quartz in sodium chloride solutions: Analysis of existing data and a rate model for $25^{\circ} \mathrm{C}$, Geochimica et Cosmochimica Acta, 56, 4147-4156.

Dove, P.M., N. Han, and J.J. De Yoreo 2005. Mechanisms of classical crystal growth theory explain quartz and silicate dissolution behavior, Proceedings of the National Academy of Sciences, 102(43), 15357-15363.

Du, J., and A.N. Cormack 2004. The medium range structure of sodium silicate glasses: a molecular dynamics simulation, Journal of Non-Crystalline Solids, 349, 66-79. 
Du, J., and A.N. Cormack 2005. Molecular dynamics simulation of the structure and hydroxylation of silica glass surfaces, Journal of American Ceramic Society, 88, 2532-2539.

Du, Z., and N.H. de Leeuw 2006. Molecular dynamics simulations of hydration, dissolution and nucleation processes at the a-quartz (0001) surface in liquid water, Dalton Transactions, 2006, 2623-2634.

Dzombak, D.A., and F.M. Morel 1990. Surface Complex Modeling, Hydrous Ferrous Oxide. Wiley Interscience, New York.

Eberl, D., J. Srodon, M. Kralik, B. Taylor, and Z. Peterman 1990. Ostwald ripening of clays and metamorphic minerals. Science 248, 474-477.

Edwards, H.C. 2002. SIERRA Framework Version 3: Core Services Theory and Design, SAND2002-3616, Sandia National Laboratories, Albuquerque, New Mexico.

Edwards, H.C., J.G. Arguello, R.A. Bartlett, J.F. Bouchard, G. Freeze, R. Howard, P. Knupp, M.T. McCornack, P. Schultz, A. Urbina, and Y. Wang 2010. Nuclear Energy Advanced Modeling and Simulation Waste Integrated Performance and Safety Codes (NEAMS Waste IPSC) Verification and Validation Plan (July 2010). Prepared for U.S. Department of Energy Advanced Modeling and Simulation Campaign.

Fang, Y., G.T. Yeh, and W.D. Burgos 2003. A new paradigm to model reaction-based biogeochemical processes. Water Resources Res, 39(4), 1083-1108.

Feuston, B.P., and S.H. Garofalini 1990. Water-induced relaxation of the vitreous silica surface, Journal of Applied Physics, 68, 4830-4836.

Felipe, M.A., J.D. Kubicki, and D.M. Rye 2003. Hydrogen isotope exchange kinetics between $\mathrm{H}_{2} \mathrm{O}$ and $\mathrm{H}_{4} \mathrm{SiO}_{4}$ from ab initio calculations, Geochimica et Cosmochimica Acta, 67, 1259-1276.

Ferlat, G., L. Cormier, M.H. Thibault, L. Galoisy, and G. Calas 2006. Evidence for symmetric cationic sites in zirconium-bearing oxide glasses, Physical Review B, 73.

Finite Element Heat and Mass Transfer (FEHM) 2010. Los Alamos National Laboratory FEHM Code website - http://fehm.lanl.gov/ (last accessed September 8, 2010).

Fossum, A. F., and R.M. Brannon 2004. The Sandia Geomodel Theory and User's Guide, SAND2004-3226, Sandia National Laboratories, Albuquerque, New Mexico.

Freeze, G., J.G. Arguello, R. Howard, J. McNeish, P. Schultz, and Y. Wang 2010. Challenge Problem and Milestones for: Nuclear Energy Advanced Modeling and Simulation (NEAMS) Waste Integrated Performance and Safety Codes (IPSC). Prepared for U.S. Department of Energy Advanced Modeling and Simulation Campaign. 
Freeze, G., P. Mariner, J.E. Houseworth, and J.C. Cunnane 2010b. Used Fuel Disposition Campaign Features, Events, and Processes (FEPs): FY10 Progress Report. SAND2010-5902. Sandia National Laboratories, Albuquerque, New Mexico.

Frugier, P., T. Chave, S. Gin, and J.-E. Lartigue 2009. Application of the GRAAL model to leaching experiments with SON68 nuclear glass in initially pure water, Journal of Nuclear Materials, 392, 552-567.

Frugier, P., S. Gin, Y. Minet, T. Chave, B. Bonin, N. Godin, J.-E. Lartigue, P. Jollivet, A. Ayral, L. De Windt, and G. Santarini 2008. SON68 nuclear glass dissolution kinetics: Current state of knowledge and basis of the new GRAAL model, Journal of Nuclear Materials, 380, 8-21.

Ganor, J., E. Roueff, Y. Erel, and J.D. Blum 2005. The dissolution kinetics of a granite and its minerals-Implications for comparison between laboratory and field dissolution rates, Geochimica et Cosmochimica Acta, 69(3), 607-621.

Ganster, P., M. Benoit, J.-M. Delaye, and W. Kob 2007. Structural and vibrational properties of a calcium aluminosilicate glass: classical force-fields vs. first-principles, Molecular Simulation, 33, 1093-1103.

Garrels, R.M., and C.L. Christ 1965. Solutions, Minerals, and Equilibria. Freeman, Cooper \& Co.

Gautier, J.M., E.H. Oelkers, and J. Schott 1994. Experimental study of K-feldspar dissolution rates as a function of chemical affinity at $150^{\circ} \mathrm{C}$ and $\mathrm{pH}$ 9, Geochimica et Cosmochimica Acta, $58,4549-4560$.

Gilijamse, J.J., A.J. Lock, and H.J. Bakker 2005. Dynamics of confined water molecules, Proceedings of the National Academy of Sciences of the United States of America, 102, 3023207.

Gillespie, D.T. 1976. General method for numerically simulating stochastic time evolution of coupled chemical reactions, Journal of Computational Physics, 22, 403-434.

Gin, S., I. Ribet, P. Frugier, T. Chave, F. Angeli, J. E. Lartigue, G. de Combarieu, and N. Godon 2005. Assessment of nuclear glass behavior in geological disposal conditions: current state of knowledge and recent advances, European Nuclear Conference Proceedings, Versailles, France.

Goldberg, S., L. Criscenti, D. Turner, J. Davis, and K. Cantrell 2007. Adsorption desorption processes in subsurface reactive transport modeling. Vadose Zone Journal 6, 407-435.

Goodwin, D. 2002. Cantera C++ User’s Guide. California Institute of Technology.

Grambow, B. 1987. Nuclear Waste Glass Dissolution Mechanism, Model, and Application. Report to the JSS Project 87-02, Phase I. 
Grambow, B. 2006. Nuclear waste glasses - how durable?, Elements, 2, 357-364.

Grossman, L. 2010. Breaking it down: Studies of how things fall apart may lead to materials that don't, Science News, 177(4), 18-21.

Hamilton, J.P., S.L. Brantley, C.G. Pantano, L.J. Criscenti, and J.D. Kubicki 2001. Dissolution of nepheline, jadeite and albite glasses: Toward better models for aluminosilicate dissolution, Geochimica et Cosmochimica Acta, 65, 3683-3702.

Hammond, G., P. Lichtner, and C. Lu 2007. Subsurface multiphase flow and multicomponent reactive transport modeling using high performance computing. Journal of Physics: Conference Series 78, 1-10.

Hansen, F., E. Hardin, R. Rechard, G. Freeze, D. Sassani, P. Brady, M. Stone, M. Martinez, J. Holland, T. Dewers, K. Gaither, S. Sobolik, and R. Cygan 2010. Shale Disposal of U.S. HighLevel Radioactive Waste, SAND2010-2843, Sandia National Laboratories, Albuquerque, New Mexico.

Harvie, C.E., N. Moller, and J.H. Weare 1984. The prediction of mineral solubilities in natural waters: The Na-K-Mg-Ca-H-Cl-SO4-OH-HCO3-CO3-CO2-H2O system to high ionic strengths at $25^{\circ} \mathrm{C}$, Geochimica et Cosmochimica Acta, 48, 723-751.

Harvie, C.E., and J.H. Weare 1980. The prediction of mineral solubilities in natural waters: The $\mathrm{Na}-\mathrm{K}-\mathrm{Mg}-\mathrm{Ca}-\mathrm{Cl}-\mathrm{SO} 4-\mathrm{H} 2 \mathrm{O}$ system from zero to high concentration at $25^{\circ} \mathrm{C}$, Geochimica et Cosmochimica Acta, 44, 981-997.

Hayes, K.F., and J.O. Leckie 1987. Modeling ionic strength effects on cation adsorption at hydrous oxide/solution interfaces, Journal of Colloid and Interface Science, 115, 564-572.

Hellmann, R., and D. Tisserand 2006. Dissolution kinetics as a function of the Gibbs free energy of reaction: An experimental study based on albite feldspar, Geochimica et Cosmochimica Acta, $70,364-383$.

Hellmann, R., C.M. Eggleston, M.F. Hochella, Jr., and D.A. Crerar 1990. The formation of leached layers on albite surfaces during dissolution under hydrothermal conditions, Geochimica Cosmochimica Acta, 54, 1267-1281.

Hellmann, R., J.-M. Penisson, R.L. Hervig, J.-H. Thomassin, and M.-F. Abrioux 2003. An EFTEM/HRTEM high-resolution study of the near surface of labradorite feldspar altered at acid pH: evidence for interfacial dissolution-precipitation, Physics and Chemistry of Minerals, 30, 192-197.

Heroux, M., A. Salinger, R. Bartlett, H. Thornquist, V. Howle, R. Tuminaro, R. Hoekstra, J. Willenbring, J. Hu, A. Williams, T. Kolda, R. Lehoucq, K. Long, R. Pawlowski, E. Phipps, and K. Stanley 2005. An overview of the TRILINOS project, ACM Transactions on Mathematical Software 31, 397-423. 
Herrmann, W., W.R. Wawersik, and H.S. Lauson 1980. Analysis of Steady State Creep of Southeastern New Mexico Bedded Salt, SAND80-0558, Sandia National Laboratories, Albuquerque, New Mexico.

Hochella, M.F., and A.F. White 1990. Mineral-water interface geochemistry: an overview, in Mineral-Water Interface Geochemistry, Reviews in Mineralogy Vol. 23, M.F. Hochella and A.F. White, eds., Mineralogical Society of America, 1-16.

Icenhower, J. and P.M. Dove 2000. The dissolution kinetics of amorphous silica into sodium chloride solutions: Effects of temperature and ionic strength, Geochimica et Cosmochimica Acta, 64, 4193-4203.

Icenhower, J.P., A. Luttge, B.P. McGrail, M.S. Beig, R.S. Arvidson, E.A. Rodriguez, J.L. Steele, and S.R. Baum 2003. Results of vertical scanning interferometry (VSI) of dissolved borosilicate glass: evidence for variable surface features and global surface retreat, Materials Research Society Symposium Proceedings, 757, II5.1.1-II5.1.8.

Ispas, S., T. Charpentier, F. Mauri, and D.R. Neuville 2010. Structural properties of lithium and sodium tetrasilicate glasses: Molecular dynamics simulations versus NMR experimental and first-principles data, Solid State Sciences, 12, 183-192.

Itasca 2008. Fast Lagrangian Analysis of Continua in Three Dimensions User's Guide, FLAC ${ }^{3 D}$ Version 4.0, Itasca Consulting Group Inc., Minneapolis, MN.

Johnson, J.W., E.H. Oelkers, and H.C. Helgeson 1992. SUPCRT92: A software package for calculating the standard molal thermodynamic properties of minerals, gases, aqueous species and reactions from 1 to 5000 bars and 0 to 1000 degrees C: Computers and Geosciences 18, 899948.

Johnson, K., A.Z. Panagiotopoulos, and K.E. Gubbins 1994. Reactive canonical Monte-Carlo a new simulation technique for reacting or associating fluids, Molecular Physics, 81, 717-733.

Kalinichev A. 2004. Personal communication, Molecular Modeling Applied to Environmental Geochemistry, ACS-PRF Summer School.

Kharaka, Y.K., and I. Barnes 1973. SOLMNEQ: solution-mineral equilibrium computations. NTIS Technical Report PB 214-899.

Knauss, K.G., and T.J. Wolery 1988. The Dissolution kinetics of quartz as a function of $\mathrm{pH}$ and time at 70-degrees-C, Geochimica et Cosmochimica Acta, 52, 43-53.

Knoll, D.A., and D.E. Keyes 2004. Jacobian-free Newton-Krylov methods: a survey of approaches and applications, Journal of Computational Physics 193, 357-397. 
Krieg, R.D. 1984. Reference Stratigraphy and Rock Properties for the Waste Isolation Pilot Plant (WIPP) Project, SAND83-1908, Sandia National Laboratories, Albuquerque, New Mexico.

Krieg, R.D. 1978. A Simple Constitutive Description for Cellular Concrete, SC-DR-72-0883, Sandia National Laboratories, Albuquerque, New Mexico.

Kubicki, J.D., G.A. Blake, and S.E. Apitz 1997. Molecular orbital calculations for modeling acetate-aluminosilicate adsorption and dissolution reactions, Geochimica et Cosmochimica Acta, 61, 1031-1046.

Kubicki, J.D. 2001. Integral equation formalism polarized continuum model calculations of aqueous $\mathrm{Al}^{3+}, \mathrm{Fe}^{3+}$, and $\mathrm{Si}^{4+}$ : Correlations of calculated aqueous-phase deprotonation energies with experimental $\ln \left(\mathrm{K}_{\mathrm{a}}\right)$ and $\mathrm{pK}_{\mathrm{a}}$ values, Journal of Physical Chemistry A, 105, 8756-8762.

Kubicki, J.D. 2008. Transition state theory and molecular orbital calculations applied to rates and rate mechanisms in geochemical kinetics in Kinetics of Water-Rock Interaction, edited by S. L. Brantley, J. D. Kubicki and A. F. White, pp. 39-72, Springer-Science.

Lasaga, A.C., and A. Luttge 2001. Variation of crystal dissolution rate based on a dissolution stepwave model, Science, 291, 2400-2404.

Lasaga, A.C., and A. Luttge 2003. A model for crystal dissolution, European Journal of Mineralogy, 2003, 603-615.

Lasaga, A.C., and A. Luttge 2004a. Mineralogical approaches to fundamental crystal dissolution kinetics, American Mineralogist, 89, 527-540.

Lasaga, A.C., and A. Luttge 2004b. Mineralogical approaches to fundamental crystal dissolution kinetics - Dissolution of an $\mathrm{A}_{3} \mathrm{~B}$ structure, European Journal of Mineralogy, 16, 713-729.

Lasaga, A.C., and A. Luttge 2005. Kinetic justification of the solubility product: application of a general kinetic dissolution model, Journal of Physical Chemistry B, 109, 1635-1642.

Lauffenberger, D. 1991. Quantitative studies of bacterial chemotaxis and microbial population dynamics. Microbial Ecology 22, 175-185.

Lee, S.K. and J.F. Stebbins 2003. O atom sites in natural kaolinite and muscovite: O-17 MAS and 3QMAS NMR study, American Mineralogist, 88, 493-500.

Leung, K., I.M.B. Nielsen, and L.J. Criscenti 2009. Elucidating the bimodal acid-base behavior of the water-silica interface from first principles, Journal of the American Chemical Society, 131(51), 18358-18365.

Li, L., C.I. Steefel, and L. Yang 2008. Scale dependence of mineral dissolution rates within single pores and fractures, Geochimica et Cosmochimica Acta, 72, 360-377. 
Lichtner, P.C. 1996. Continuum formulation of multicomponent-multiphase reactive transport, in Reactive Transport in Porous Media, Reviews in Mineralogy Vol. 34, Mineralogical Society of America, 1-79.

Lichtner, P.C., C.I. Steefel, and E.H. Oelkers, eds. 1996. Reactive Transport in Porous Media, Reviews in Mineralogy Vol. 34, Mineralogical Society of America.

Lichtner, P.C. 1988. The quasi-stationary state approximation to coupled mass transport and fluid-rock interaction in a porous media, Geochimica et Cosmochimica Acta 52, 143-165.

Lin, J.I. 2005. DYNA3D : A Nonlinear, Explicit, Three-dimensional Finite Element Code for Solid and Structural Mechanics, User's Manual, UCRL-MA-107254, Lawrence Livermore National Laboratory, Livermore, CA.

Livermore Software Technology Corporation (LTSC) 2003. LS-DYNA Keyword User's Manual, Version 970, Livermore Software Technology Corporation, Livermore, CA.

Luttge, A., and R.S. Arvidson 2008. The mineral-water interface in Kinetics of Water-Rock Interaction, edited by S. L. Brantley, J. D. Kubicki and A. F. White, pp. 73-107, SpringerScience.

Luttge, A., E.W. Boltin, and A.C. Lasaga 1999. An interferometric study of the dissolution kinetics of anorthite: the role of reactive surface area. American Journal of Science, 299, 652678.

Luttge, A., U. Winkler, and A.C. Lasaga 2003. Dolomite dissolution kinetics studied with vertical scanning white light interferometry. Geochimica et Cosmochimica Acta, 67, 1099-1116.

Luttge, A. 2004. Crystal dissolution kinetics studied by Vertical Scanning Interferometry (VSI) and Monte Carlo simulations: a brief review and outlook in Nanoscale Structure and Assembly at Solid-Fluid Interfaces. Vol. I. Interfacial Structures Versus Dynamics, edited by X.-Y. Liu and J. J. De Yoreo, Series on Nanoscience and Technologies, Kluwer, New York, 700 pp.

MacQuarrie, K., and K.U. Mayer 2005. Reactive transport modeling in fractured rock: A stateof-the-science review. Earth Science Reviews 72, 189-227.

Maker, B.N. 1995. NIKE3D: A Nonlinear, Implicit, Three-dimensional Finite Element Code for Solid and Structural Mechanics, User's Manual, UCRL-MA-105268, Lawrence Livermore National Laboratory, Livermore, CA.

Merino, E. 1975. Diagenesis in Tertiary sandstones from Kettleman North Dome, California II. Interstitial solutions: distribution of aqueous species at $100^{\circ} \mathrm{C}$ and chemical relation to the diagenetic mineralogy. Geochimica et Cosmochimica Acta 39, 1629-1645. 
Meshri, I. 1990. An overview of chemical models and their relationship to porosity prediction in the subsurface in Prediction of Reservoir Quality Through Chemical Modeling, I. Meshri and P. Ortoleva, eds, AAPG Memoir 49, pp. 45-53.

Mills, R.T., C. Lu, P.C. Lichtner, and G.E. Hammond 2007. Simulating subsurface flow and transport on ultrascale computers using PFLOTRAN, Journal of Physics: Conference Series, $v$. 78 (Proceedings of SciDAC 2007). DOI 10.1088/1742-6596/78/1/012051.

Morrow, C.P., S. Nangia, and B.J. Garrison 2009. Ab initio investigation of dissolution mechanisms in aluminosilicate minerals, Journal of Physical Chemistry A, 113, 1343-1352.

Munson, D.E., A.F. Fossum, and P.E. Senseny 1989. Advances in Resolution of Discrepancies Between Predicted and Measured In-Situ WIPP Room Closures, SAND88-2948, Sandia National Laboratories, Albuquerque, New Mexico.

Nationale Genossenschaft für die Lagerung Radioactiver Abfälle [National Cooperative for the Disposal of Radioactive Waste] (NAGRA) 2002. Project Opalinus Clay Safety Report: Demonstration of disposal feasibility for spent fuel, vitrified high-level waste and long-lived intermediate-level waste (Entsorgungsnachweis), Technical Report 02-05.

Nangia, S., and B.J. Garrison 2008. Reaction rates and dissolution mechanisms of quartz as a function of pH, Journal of Physical Chemistry A, 112, 2027-2033.

Nangia, S., and B.J. Garrison 2009. Advanced Monte Carlo approach to study evolution of quartz surface during the dissolution process, Journal of American Chemical Society, 131, 95389546.

Nangia, S., and B.J. Garrison 2010a. Role of intrasurface hydrogen bonding on silica dissolution, Journal of Physical Chemistry C, 114, 2267-2272.

Nangia, S. and B.J. Garrison. 2010b. Theoretical advances in the dissolution studies of mineralwater interfaces, Theoretical Chemistry Accounts, DOI 10.1007/s00214-010-0770-2.

Nangia, S., N.M. Washton, K.T. Mueller, J.D. Kubicki, and B.J. Garrison 2007. Study of a family of 40 hydroxylated $ß$-cristobalite surfaces using empirical potential energy functions, Journal of Physical Chemistry C, 111, 5169-5177.

Nordstrom, D.K., L.N. Plummer, T.M.L. Wigley, T.J. Wolery, J.W. Ball, E.A. Jenne, R.L. Bassett, D.A. Crerar, T.M. Florence, B. Fritz, M. Hoffman, G.R. Holdren, Jr., G.M. Lafon, S.V. Mattigod, R.E. McDuff, F. Morel, M.M. Reddy, G. Sposito, and J. Thrailkill 1979. A comparison of computerized chemical models for equilibrium calculations in aqueous systems: in Chemical Modeling in aqueous systems, speciation, sorption, solubility, and kinetics, E.A. Jenne, ed., Series 93, American Chemical Society, pp. 857-892. 
Noorishad, J., C.L. Carnahan, and L.V. Benson 1987. Development of the non-equilibrium reactive chemical transport code CHMTRNS. Lawrence Berkeley National Laboratory, Earth Science Division, Berkeley CA.

Notz, P., S. Subia, M. Hopkins, H. Moffat, and D. Noble 2007. ARIA 1.5 User's Manual, SAND2007-2734, Sandia National Laboratories, Albuquerque, New Mexico.

Oelkers, E.H. 2001. General kinetic description of multioxide silicate mineral and glass dissolution, Geochimica et Cosmochimica Acta, 65, 3703-3719.

Oelkers, E.H. and S.R. Gislason 2001. The mechanism, rates and consequences of basaltic glass dissolution. Geochimica et Cosmochimica Acta 65, 3671-3681.

Oelkers, E.H., J. Schott, and J.L. Devidal 1994. The effect of aluminum, pH, and chemical affinity on the rates of aluminosilicate dissolution reactions, Geochimica et Cosmochimica Acta, 58, 2011-2024.

Ong, S., X. Zhao, and K.B. Eisenthal 1992. Polarization of water molecules at a charged interface: second harmonic studies of the silica/water interface, Chemical Physics Letters, 191, 327-335.

Ortoleva, P., G. Auchmuthy, J. Chadam, J. Hettmer, E. Merino, C. Moore, and E. Ripley 1986. Redox front propagation and banding modalities. Physica, 19D, 334-354.

Ortoleva, P., E. Merino, C. Moore, and J. Chadam 1987. Geochemical self-organization I: reaction-transport feedbacks and modeling approach. American Journal of Science 287, 9791007.

Ortoleva, P., J. Chadam, E. Merino, and A. Sen 1987. Geochemical self-organization II: the reactive infiltration instability. American Journal of Science 287, 1008-1040.

Palandri, J., and Y. Kharaka 2004. A complilation of rate parameters of water-mineral interaction kinetics for application to geochemical modeling. USGS Open-File Report 20041068 .

Parkhurst, D.L., D.C. Thorstenson, and L.N. Plummer 1980. PHREEQE - a computer program for geochemical calculations. USGS Water Resources Investigations Report 80-96.

Parkhurst, D.L. and C.A.J. Appelo 1999. User's Guide to PHREEQC (Version 2) - A computer program for speciation, batch reaction, one-dimensional transport, and inverse geochemical calculation. USGS Water Resources Investigations Report 99-4259.

Parkhurst, D.L., K.L. Kipp, P. Engesgaard, and S.R. Charlton 2004. PHAST-A program for simulating ground-water flow, solute transport, and multicomponent geochemical reactions: U.S. Geological Survey Techniques and Methods 6-A8. 
Pedone, A., G. Malavasi, M.C. Menziani, A.N. Cormack, and U. Segre 2006. A new selfconsistent empirical interatomic potential model for oxides, silicates, and silica-based glasses, Journal of Physical Chemistry B, 110, 11780-11795.

Pedone, A., G. Malavasi, A.N. Cormack, U. Segre, and M.C. Menziani 2008. Elastic and dynamical properties of alkali-silicate glasses from computer simulations techniques, Theoretical Chemistry Accounts, 120, 557-564.

Pelmenschikov, A., H. Strandh, L.G.M. Pettersson, and J. Leszcyzynski 2000. Lattice resistance to hydrolysis of Si-O-Si bonds of silicate minerals: $\mathrm{Ab}$ initio calculations of a single water attack onto the (001) and (111) B-cristobalite surfaces, Journal of Physical Chemistry B, 5779-5783.

Pelmenschikov, A., J. Leszczynski, and L.G.M. Pettersson 2001. Mechanism of dissolution of neutral silica surfaces: Including effect of self-healing, Journal of Physical Chemistry A, 105, 9528-9532.

Pierce, E.M., B.P. McGrail, M.M. Valenta, and D.M. Strachan 2006. The accelerated weathering of a radioactive low-activity waste glass under hydraulically unsaturated conditions: Experimental results from a pressurized unsaturated flow test, Nuclear Technology, 155, 149165.

Pierce, E.M., L.R. Reed, W.J. Shaw, B.P. McGrail, J.P. Icenhower, C.F. Windisch, E.A. Cordova, and J. Broady 2010. Experimental determination of the effect of the ratio of $\mathrm{B} / \mathrm{Al}$ on glass dissolution along the nepheline $\left(\mathrm{NaAlSiO}_{4}\right)$-malinkoite $\left(\mathrm{NaBSiO}_{4}\right)$ join, Geochimica et Cosmochimica Acta, 74, 2634-2654.

Pitzer, K.S. 1979. Theory-ion interaction approach in Activity Coefficients in Electrolyte Solutions, v. 1, R.M. Pytkowicz, ed., CRC Press, Boca Raton, Florida, pp. 157-208.

Plummer, L.N., D.L. Parkhurst, and D. Kosiur 1975. MIX2: A computer program for modeling chemical reactions in natural waters. USGS Water Resources Investigations 75-61.

Plummer, L.N., D.L. Parkhurst, G.W. Fleming, and S.A. Dunkle 1988. A computer program incorporating Pitzer's equations for calculation of geochemical reactions in brines. USGS Water Resources Investigations Report 88-4153.

Prommer, H., G.B. Davis, and D.A. Barry 1999b. PHT3D - A three dimensional biogeochemical transport model for modelling natural and enhanced remediation in Proc. contaminated site remediation: Challenges posed by urban and industrial contaminants, C.D. Johnston, ed., Fremantle, Western Australia, 21-25 March 1999, pp. 351-358.

Pruess, K., C. Oldenburg, and G. Moridis 1999. TOUGH2 User's Guide, Version 2.0, LBL43134, Lawrence Berkeley National Laboratory, Berkeley, California.

Ramkrishna, D. 2000. Population Balances: Theory and Applications to Particulate Systems in Engineering, Academic Press. 
Ramshaw, J.D. 1980. Partial equilibrium in fluid dynamics. Phys. Fluids 23, 675-680.

Randolph, A.D., and M.A. Larson 1988. Theory of Particulate Processes. Academic Press.

Reed, M. 1982. Calculation of multicomponent chemical equilibria and reaction processes in systems involving minerals, gases, and an aqueous phase. Geochimica et Cosmochimica Acta 46, 513-528.

Reeves, H., and D.J. Kirkner 1988. Multicomponent mass transport with homogeneous and heterogeneous chemical reactions: effect of chemistry on the choice of numerical algorithm. 2. numerical results. Water Resources Research 24, 1730-1739.

Rubin, J. and R.V. James 1973. Dispersion-affected transport of reacting solutes in saturated porous media: Galerkin method applied to equilibrium controlled exchange in unidirectional steady water flow. Water Resources Research 9(5), 1332-1352.

Sandia National Laboratories (SNL) 2009. Waste Forms and Disposal Systems Integrated Performance and Safety Codes: System Design Specification. Prepared for U.S. Department of Energy Advanced Modeling and Simulation Campaign.

Schechter, R.S., S.L. Bryant, and L.W. Lake 1987. Isotherm-free chromatography: propagation of precipitation/dissolution waves. Chemical Engineering Communications 58, 353-376.

Schwartz, F., and H. Zhang 2003. Fundamentals of Groundwater, John Wiley and Sons.

Schwartzentruber, J., W. Furst, and H. Renon 1987. Dissolution of quartz into dilute alkalinesolutions at 90-degrees-C - A kinetic study, Geochimica et Cosmochimica Acta, 51, 1867-1874.

Siepmann, J.I., and D. Frenkel 1992. Configurational bias Monte Carlo: A new sampling scheme for flexible chains, Molecular Physics, 75, 59-70

SIERRA Solid Mechanics Team 2010a. Presto 4.18 User's Guide, SAND2010-3112, Sandia National Laboratories, Albuquerque, New Mexico.

SIERRA Solid Mechanics Team 2010b. Adagio 4.16 User's Guide, SAND2010-3111, Sandia National Laboratories, Albuquerque, New Mexico.

SIMULIA 2007. Abaqus Analysis User’s Manual, Version 6.7, SIMULIA, Inc., Providence, RI.

Simunek, J., M. van Genuchten, and M. Sejna 2008. Development and application of the HYDRUS and STANMOD software packages and related codes. Vadose Zone Journal 7, 587600 . 
Sjaardema, G.D., and R.D. Krieg 1987. A Constitutive Model for the Consolidation of WIPP Crushed Salt and Its Use in Analyses of Backfilled Shaft and Drift Configurations, SAND871977, Sandia National Laboratories, Albuquerque, New Mexico.

Smith, W.R., and B. Triska 1994. The reaction ensemble method for the computer simulation of chemical and phase equilibria. I. Theory and basic examples, Journal of Chemical Physics, 100, 3019-3027.

Strachan, D.M., and T.L. Croak 2000. Compositional effects on long-term dissolution of borosilicate glass, Journal of Non-Crystalline Solids, 272, 22-33.

Steefel, C.I., and P. Van Cappellan 1990. A new kinetic approach to modeling water-rock interaction: The role of nucleation, precursors, and Ostwald ripening. Geochimica et Cosmochimica Acta 54, 2657-2677.

Steefel, C.I., and K.T.B MacQuarrie 1996. Approaches to modeling of reactive transport in porous media in Reactive Transport in Porous Media, Reviews in Mineralogy Vol. 34, Mineralogical Society of America, 83-129.

Steefel, C.I. 2001. CRUNCH, Lawrence Livermore National Laboratory, Livermore CA.

Steefel, C.I., D.J. DePaolo, and P.C. Lichtner 2005. Reactive transport modeling: An essential tool and a new research approach for the Earth Sciences, Earth and Planetary Science Letters, 240, 539-558.

Stewart, J., and C. Edwards 2004. A framework approach for developing parallel adaptive multiphysics applications. Finite Elements in Analysis and Design 40, 1599-1617.

Stone, C.M., J.F. Holland, J.E. Bean, and J.G. Arguello 2010. Coupled thermal-mechanical analyses of a generic salt repository for high level waste, ARMA 10-180, American Rock Mechanics Association, Proceedings of the $44^{\text {th }}$ U. S. Rock Mechanics Symposium, Salt Lake City, UT, June 27-30, 2010.

Svensk Kämbränslehantering $\mathrm{AB}$ [Swedish Nuclear Fuel and Waste Management Co.] (SKB) 2006. Long-term Safety for KBS-3 Repositories at Forsmark and Laxemar-a First Evaluation, Technical Report TR-06-09.

Swift, P.N., C.W. Hansen, E. Hardin, R.J. MacKinnon, D. Sassani, and S.D. Sevougian 2010. Potential Impacts of Alternative Waste Forms on Long-Term Performance of Geological Repositories for Radioactive Waste, Proceedings of 10th International Probabilistic Safety Assessment Management (PSAM) Conference, Seattle WA, June 7-11, 2010.

Taylor, L.M., and D.P. Flanagan 1989. Pronto 3D A Three-Dimensional Transient Solid Dynamics Program, SAND87-1912, Sandia National Laboratories, Albuquerque, New Mexico. 
Tilocca, A., N.H. de Leeuw, and A.N. Cormack 2006. Shell-model molecular dynamics calculations of modified silicate glasses, Physical Review B, 73.

Tilocca, A., A.N. Cormack, and N.H. de Leeuw 2007. The structure of bioactive silicate glasses: New insight from molecular dynamics simulations, Chemistry of Materials, 19, 95-103.

Tilocca, A., and A.N. Cormack 2008. Exploring the surface of bioactive glasses: Water adsorption and reactivity, Journal of Physical Chemistry C, 112, 11936-11945.

Tossell, J.A., and G. Saghi-Szabo 1997. Aluminosilicate and borosilicate single 4-rings: Effects of counterions and water on structure, stability, and spectra, Geochimica et Cosmochimica Acta, 61, 1171-1179.

Truesdell, A.H., and B.F. Jones 1974. WATEQ, A computer program for calculating chemical equilibria of natural waters: Journal of Research, U.S. Geological Survey, v. 2, 233-274.

Tsomaia, N., S.L. Brantley, J.P. Hamilton, C.G. Pantano, and K.T. Mueller 2003. NMR evidence for formation of ocathedral and tetrahedral $\mathrm{Al}$ and repolymerization of the Si network during dissolution of aluminosilicate glass and crystal, American Mineralogist, 88, 54-67.

U.S. Department of Energy (DOE) 2009. Yucca Mountain Repository License Application, DOE/RW-0573, Rev. 1.

Valocchi, A. and M. Malmstead 1992. Accuracy of operator splitting for advection-dispersionreaction problems. Water Resources Research 28(5): 1471-1476.

van Genuchten, M.T. 1980. A closed-form equation for predicting the hydraulic conductivity of unsaturated soils, Soil Sci. Soc., 44, 892-898.

Van Iseghem., P., K. Lemmens, M. Aertsens, S. Gin, I. Ribet, B. Grambow, J.L. Crovisier, M. Del Nero, E. Curti, B. Schwyn, B. Lucksheiter, and T. McMenamin 2006. Chemical durability of high-level waste glass in repository environment: main conclusions and remaining uncertainties from the GLASTAB and GLAMOR projects, Materials Research Society Symposium Proceedings, 932, 293-304.

Van Iseghem, P., M. Aertsens, S. Gin, D. Deneele, B. Grambow, P. McGrail, D. Strachan, and G. Wicks 2007. GLAMOR A critical evaluation of the dissolution mechanisms of high level waste glasses in conditions of relevance for geological disposal, European Commission Final Report, 2001-11-01 to 2004-10-31.

Vaughn, P. 2010. A Framework Assessment Tool. Sandia National Laboratories Internal Communication, Albuquerque, New Mexico, August 2010.

Verney-Carron, A., S. Gin, P. Frugier, and G. Libourel 2010. Long-term modeling of alterationtransport coupling: Application to a fractured Roman glass, Geochimica et Cosmochimica Acta, 74, 2291-2315. 
Walsh, M.P., S.L. Bryant, R.S. Schecter, and L.W. Lake 1984. Precipitation and dissolution of solids attending flow through porous media, AIChE Journal 30, 317-327.

Wang, Y., T. Dewers, T. Hadgu, C.F. Jove-Colon, A.C. Sun, and J. McNeish 2010. Enhanced Performance Assessment System (EPAS) for Carbon Sequestration, SAND2010-6173, Sandia National Laboratories, Albuquerque, New Mexico.

Weber, W. J., A. Navrotsky, S. Stefanovsky, E.R. Vance, and E. Vernaz 2009. Materials science of high-level nuclear waste immobilization, Materials Research Society, 34, 46-53.

Wheeler, S.J., and V. Sivakumar 1995. An elasto-plastic critical state framework for unsaturated soils, Géotechnique, 45 (1), 35-53.

White, A.F., and S.L. Brantley 2003. The effect of time on the weathering of silicate minerals: why do weathering rates differ in the laboratory and the field?, Chemical Geology, 202, 479-506.

White, M.D. and M. Oostrom 2006. STOMP - Subsurface Transport Over Multiple Phases, Version 4.0, User's Guide, PNNL-15782, Pacific Northwest National Laboratory, Richland, Washington.

Wolery, T.J. 1979. Calculation of chemical equilibrium between aqueous solution and minerals: the EQ 3/6 software package. UCRL-52658. Lawrence Livermore National Laboratory, Livermore CA.

Wolery, T.J. 1992. EQ3NR, a computer program for geochemical aqueous speciation-solubility calculations: theoretical manual, user's guide, and related documentation (Version 7). UCRLMA-110662 PT III. Lawrence Livermore National Laboratory, Livermore CA.

Wolery, T.J. 1992. EQ3/6: Software package for geochemical modeling of aqueous systems: package overview and installation guide (version 8.0), UCRL-MA-110662 PT I, Lawrence Livermore National Laboratory, Livermore, CA.

Wolery, T.W. and R.L. Jarek 2003. EQ3/6, Version 8.0, Software User's Manual, U.S. Department of Energy, Office of Civilian Radioactive Waste Management, Office of Repository Development, Las Vegas, Nevada.

Wolf-Boenisch, D., S.R. Gislason, E.H. Oelkers, and C. V. Putnis 2004. The dissolution rates of natural glasses as a function of their composition at $\mathrm{pH} 4$ and 10.6, and temperatures from 25 to $74^{\circ} \mathrm{C}$, Geochimica et Cosmochimica Acta, 68(23), 4843-4858.

Wollast, R., and L. Chou 1988. Rate control of silicate minerals at room temperature and pressure, in Proceedings of the NATO Advanced Study Institute on Physical and Chemical Weathering in Geochemical Cycles edited by A. Lerman and M. Meybeck, pp. 11-32, Kluwer Academic Publishers, Geneva. 
Xiao, Y., and A.C. Lasaga 1994. Ab inito quantum mechanical studies of the kinetics and mechanisms of silicate dissolution: $\mathrm{H}^{+}\left(\mathrm{H}_{3} \mathrm{O}^{+}\right)$catalysis, Geochimica et Cosmochimica Acta, 58, 5379-5400.

Xiao, Y., and A.C. Lasaga 1996. Ab initio quantum mechanical studies of the kinetics and mechanisms of quartz dissolution: $\mathrm{OH}^{-}$catalysis, Geochimica et Cosmochimica Acta, 60, 22832295.

Xu, T., E. Sonnenthal, N. Spycher, and K. Pruess 2004. TOUGHREACT User's Guide: A simulation program for non-isothermal multiphase reactive geochemical transport in variable saturated geologic media. LBNL-55460, Lawrence Berkeley National Laboratory, Berkeley, CA.

Yang, L., and C.I. Steefel 2008. Kaolinite dissolution and precipitation kinetics at $22{ }^{\circ} \mathrm{C}$ and $\mathrm{pH}$ 4, Geochimica et Cosmochimica Acta, 72, 99-116.

Yeh, G.T., and V.S. Tripathi 1989. A critical evaluation of recent developments in hydrogeochemical transport models of reactive multichemical components. Water Resources Research 25, 93-108.

Yeh, G.T. and V.S. Tripathi 1990. HYDROGEOCHEM: A Coupled Model of HYDROlogical Transport and GEOCHEMical Equilibrium of Multicomponent Systems, ORNL-6371, Oak Ridge National Laboratory, Oak Ridge, TN.

Yeh, G.T., J.T. Sun, P.M. Jardine, W.D. Burger, Y.L. Fang, M.H. Li, and M.D. Siegel $2004 b$. HYDROGEOCHEM 5.0: A Three-Dimensional Model of Coupled Fluid Flow, Thermal Transport, and HYDROGEOCHEMical Transport through Variably Saturated Conditions Version 5.0. ORNL/TM-2004/107, Oak Ridge National Laboratory, Oak Ridge, TN.

Yu, P., A.P. Lee, B.L. Phillips, and W.H. Casey 2003. Potentiometric and F-19 nuclear magnetic resonance spectroscopic study of fluoride substitution in the GaAl12 polyoxocation: Implications for aluminum (hyr)oxide mineral surfaces. Geochimica et Cosmochimica Acta, 67, 1065-1080.

Yuan, X., and A.N. Cormack 2001. Local structures of MD-modeled vitreous silica and sodium silicate glasses, Journal of Non-Crystalline Solids, 283, 69-87.

Zeitler, T.R., and A.N. Cormack 2006. Interaction of water with bioactive glass surfaces, Journal of Crystal Growth, 294, 96-102.

Zhang, K., Y.-S. Wu, and K. Pruess 2008. User's Guide for TOUGH2-MP - A Massively Parallel Version of the TOUGH2 Code, LBNL-315E, Lawrence Berkeley National Laboratory, Berkeley, CA.

Zhang, L., and A. Luttge 2007. Al,Si order in albite and its effect on albite dissolution processes: A Monte Carlo study, American Mineralogist, 92, 1316-1324. 
Zhang, L., and A. Luttge 2008. Aluminosilicate dissolution kinetics: a general stochastic model, Journal of Physical Chemistry B, 112, 1736-1742.

Zhang, L., and A. Lüttge 2009. Theoretical approach to evaluating plagioclase dissolution mechanisms, Geochimica et Cosmochimica Acta, 73(10), 2832-2849.

Zyvoloski, G. 2007. FEHM: A control volume finite element code for simulating subsurface multiphase multi-fluid heat and mass transfer. LA-UR-07-3359, Los Alamos National Laboratory, Los Alamos, New Mexico. 


\section{APPENDIX A: REACTIVE TRANSPORT CODES}

Table A-1 lists reactive transport codes with certain THC capabilities that are potentially relevant to the Waste IPSC. 
Table A-1. Reactive Transport Codes with THC Capabilities Potentially Relevant to the Waste IPSC

\begin{tabular}{|c|c|c|c|c|c|c|c|c|c|c|c|c|c|c|}
\hline \multirow{3}{*}{\multicolumn{2}{|c|}{ Model Name }} & \multirow{4}{*}{$\begin{array}{l}\text { Dim. } \\
1 \mathrm{D} / \\
2 \mathrm{D} / \\
3 \mathrm{D}\end{array}$} & \multirow{4}{*}{$\begin{array}{c}\text { Flow } \\
\text { MF }\end{array}$} & \multicolumn{6}{|c|}{ Processes and Approaches } & \multirow{4}{*}{$\begin{array}{c}\text { Activity models } \\
\text { Davies, } \\
\text { extended Debye- } \\
\text { Huckel (B-dot) }\end{array}$} & \multirow{4}{*}{$\begin{array}{c}\begin{array}{c}\text { Sorption } \\
\text { models }\end{array} \\
\text { SCM? + IE }\end{array}$} & \multirow{4}{*}{$\begin{array}{l}\text { Solver } \\
\text { Appr. } \\
\text { SNIA }\end{array}$} & \multirow{4}{*}{$\begin{array}{l}\text { Num. } \\
\text { Meth. } \\
\text { FD }\end{array}$} & \multirow{4}{*}{$\begin{array}{l}\text { Notes } \\
\text { Includes colloid transport. }\end{array}$} \\
\hline & & & & \multirow{3}{*}{$\begin{array}{c}\begin{array}{c}\text { Ther- } \\
\text { mal }\end{array} \\
\text { Yes }\end{array}$} & \multirow{3}{*}{ 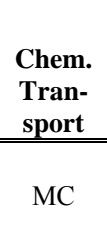 } & \multicolumn{4}{|c|}{ Reactions } & & & & & \\
\hline & & & & & & $\begin{array}{l}\text { Aq. } \\
\text { Com- } \\
\text { plex }\end{array}$ & Sorp & $\mathbf{P} / \mathbf{D}$ & \multirow{2}{*}{ Bio } & & & & & \\
\hline \multirow{2}{*}{$\begin{array}{l}\text { FLOTRAN } \\
\text { family }\end{array}$} & $\begin{array}{l}\text { FLOTRAN } \\
\text { (LANL) }\end{array}$ & & & & & EK & EK & EK & & & & & & \\
\hline & $\begin{array}{l}\text { PFLOTRAN } \\
\text { (LANL) }\end{array}$ & $\begin{array}{l}1 \mathrm{D} / \\
2 \mathrm{D} / \\
3 \mathrm{D}\end{array}$ & MF & Yes & $\mathrm{MC}$ & EK? & EK? & EK & No? & $?$ & $?$ & $?$ & FV & $\begin{array}{l}\text { Massively parallel. Based on earlier } \\
\text { FLOTRAN. }\end{array}$ \\
\hline \multirow{4}{*}{$\begin{array}{l}\text { CORE } \\
\text { family }\end{array}$} & $\mathrm{CORE}^{2 \mathrm{D}}$ V.2 & $\begin{array}{l}2 \mathrm{D} / \\
3 \mathrm{D}^{\mathrm{a}}\end{array}$ & VF & Yes & $\mathrm{MC}$ & EQ & EQ & EK & No & $\begin{array}{l}\text { extended Debye- } \\
\text { Huckel (B-dot) }\end{array}$ & $\begin{array}{l}\text { DLM, LI, } \\
\quad \text { IE }\end{array}$ & SIA & $\mathrm{FE}$ & $\begin{array}{l}\text { Based in part on the earlier code } \\
\text { TRANQUI. }\end{array}$ \\
\hline & $\begin{array}{l}\text { FADES- } \\
\text { CORE, } \\
\text { INVERSE- } \\
\text { FADES- } \\
\text { CORE } \\
\end{array}$ & $\begin{array}{l}2 \mathrm{D} / \\
3 \mathrm{D}^{\mathrm{a}}\end{array}$ & VF & Yes & $\mathrm{MC}$ & EQ & EQ & EK & No & $\begin{array}{l}\text { extended Debye- } \\
\text { Huckel (B-dot) }\end{array}$ & $\begin{array}{l}\text { DLM, LI, } \\
\quad \text { IE }\end{array}$ & SIA & $\mathrm{FE}$ & $\begin{array}{l}\text { THMC code, created by coupling CORE } \\
\text { version } 2 \text { with FADES, a soil THM code. } \\
\text { An inverse-modeling version (INVERSE- } \\
\text { FADES-CORE) is available. }\end{array}$ \\
\hline & $\begin{array}{l}\text { Bio-CORE } \\
\text { (LBNL) } \\
\text { (Zhang } \\
2001)\end{array}$ & $\begin{array}{l}2 \mathrm{D} / \\
3 \mathrm{D}^{\mathrm{a}}\end{array}$ & VF & Yes & $\mathrm{MC}$ & EQ & EQ & EK & Yes & $\begin{array}{l}\text { extended Debye- } \\
\text { Huckel (B-dot), } \\
\text { Pitzer }\end{array}$ & $\begin{array}{l}\text { DLM, LI, } \\
\text { IE }\end{array}$ & SIA & $\mathrm{FE}$ & $\begin{array}{l}\text { Based on CORE }{ }^{2 \mathrm{D}} \text { V. } 2 \text {. Implements a large } \\
\text { suite of biological processes. One version } \\
\text { of Bio-CORE }{ }^{2 \mathrm{D}} \text { has implemented the Pitzer } \\
\text { model. }\end{array}$ \\
\hline & $\begin{array}{l}\text { CORE }^{2 D} \text { V.4 } \\
\text { (LBNL) } \\
\text { (Sampler et } \\
\text { al. 2003; } \\
\text { Yang and } \\
\text { Sampler } \\
\text { 2007) }\end{array}$ & $\begin{array}{l}2 \mathrm{D} / \\
3 \mathrm{D}^{\mathrm{a}}\end{array}$ & VF & Yes & $\mathrm{MC}$ & EK & EQ & EK & Yes & $\begin{array}{l}\text { extended Debye- } \\
\text { Huckel (B-dot) }\end{array}$ & $\begin{array}{l}\text { DLM, LI, } \\
\text { NLI, IE }\end{array}$ & $\begin{array}{l}\text { SIA/ } \\
\text { SNIA/ } \\
\text { SPIA }\end{array}$ & $\mathrm{FE}$ & $\begin{array}{l}\text { This version incorporates the biological } \\
\text { reactions from Bio-CORE }{ }^{2 \mathrm{D}} \text { and the inverse } \\
\text { modeling capabilities of INVERSE-CORE } \\
\text { and other changes; it does not include } \\
\text { mechanical deformation. }\end{array}$ \\
\hline
\end{tabular}


Table A-1. Reactive Transport Codes with THC Capabilities Potentially Relevant to the Waste IPSC

\begin{tabular}{|c|c|c|c|c|c|c|c|c|c|c|c|c|c|c|}
\hline & & \multirow[b]{3}{*}{ Dim. } & \multicolumn{7}{|c|}{ Processes and Approaches } & \multirow[b]{3}{*}{ Activity models } & \multirow[b]{3}{*}{$\begin{array}{c}\begin{array}{c}\text { Sorption } \\
\text { models }\end{array} \\
\end{array}$} & \multirow[b]{3}{*}{$\begin{array}{l}\text { Solver } \\
\text { Appr. }\end{array}$} & \multirow[b]{3}{*}{$\begin{array}{l}\text { Num. } \\
\text { Meth. }\end{array}$} & \multirow[b]{3}{*}{ Notes } \\
\hline & & & & & & & & & & & & & & \\
\hline \multicolumn{2}{|c|}{ Model Name } & & Flow & $\begin{array}{c}\text { Ther- } \\
\text { mal }\end{array}$ & $\begin{array}{l}\text { Cnem. } \\
\text { Tran- } \\
\text { sport }\end{array}$ & $\begin{array}{l}\text { Aq. } \\
\text { Com- } \\
\text { plex }\end{array}$ & Sorp & $\mathbf{P} / \mathbf{D}$ & Bio & & & & & \\
\hline \multirow{6}{*}{$\begin{array}{l}\text { MT3DMS } \\
\text { family }\end{array}$} & MT3DMS & $3 \mathrm{D}$ & No & $\mathrm{Yes}^{\mathrm{b}}$ & $\mathrm{MC}$ & No & Emp & No & No & N/A & LI & SNIA & $\mathrm{FD}^{*}$ & $\begin{array}{l}\text { Transport code that is designed to couple } \\
\text { readily with MODFLOW. *Also does } \\
\text { particle tracking and total-variation- } \\
\text { diminishing. }\end{array}$ \\
\hline & $\begin{array}{l}\text { RT3D } \\
\text { (PNNL) }\end{array}$ & $3 \mathrm{D}$ & No & Yes $^{b}$ & $\mathrm{MC}$ & No & Emp & No & Yes & N/A & $\begin{array}{l}\text { LI, NLI, } \\
\text { implement } \\
\text { sorption } \\
\text { kinetics }\end{array}$ & SNIA & FD & $\begin{array}{l}\text { Based on MT3DMS, requires coupling with } \\
\text { MODFLOW for flow. Used to perform } \\
\text { calculations of subsurface transport and } \\
\text { biodegradation of organic contaminants, } \\
\text { primarily in support of natural attenuation } \\
\text { studies. }\end{array}$ \\
\hline & $\begin{array}{l}\text { SEAM3D } \\
\text { (US Army } \\
\text { Engineer } \\
\text { R\&D Center) }\end{array}$ & $3 \mathrm{D}$ & No & $\mathrm{Yes}^{\mathrm{b}}$ & $\mathrm{MC}$ & No & Emp & No & Yes & N/A & LI & SNIA & FD & $\begin{array}{l}\text { Based on MT3DMS, requires coupling with } \\
\text { MODFLOW for flow. Used to perform } \\
\text { calculations of subsurface transport and } \\
\text { biodegradation of organic contaminants. }\end{array}$ \\
\hline & $\begin{array}{l}\text { SEAWAT } \\
\text { (USGS) }\end{array}$ & $3 \mathrm{D}$ & SF & Yes & $\mathrm{MC}$ & No & No & No & No & N/A & N/A & SNIA & FD & $\begin{array}{l}\text { A code SEAWAT combines MT3DMS and } \\
\text { MODFLOW under conditions of changing } \\
\text { density. Designed to model salt-water } \\
\text { intrusion into freshwater aquifers. This } \\
\text { code does consider effects of thermally- } \\
\text { driven differences in density or thermally- } \\
\text { driven flow (convection). }\end{array}$ \\
\hline & PH3TD & $3 \mathrm{D}$ & SF & $\mathrm{Yes}^{\mathrm{b}}$ & $\mathrm{MC}$ & $\mathrm{EK}^{\mathrm{c}}$ & EK & EK & No & $\begin{array}{l}\text { Davies, extended } \\
\text { Debye-Huckel }^{\mathrm{e}}\end{array}$ & DM, NEM & SNIA & FD & Based on PHREEQC V.2 and MT3DMS. \\
\hline & PHWAT & $3 \mathrm{D}$ & SF & Yes & $\mathrm{MC}$ & $\mathrm{EK}^{\mathrm{c}}$ & EK & EK & No & $\begin{array}{l}\text { Davies, extended } \\
\text { Debye-Huckel }^{\mathrm{e}}\end{array}$ & DM, NEM & SNIA & FD & $\begin{array}{l}\text { Based on coupling SEAWAT and } \\
\text { PHREEQC V.2, this code does consider } \\
\text { thermal effects on flow (e.g., convection). }\end{array}$ \\
\hline
\end{tabular}


Table A-1. Reactive Transport Codes with THC Capabilities Potentially Relevant to the Waste IPSC

\begin{tabular}{|c|c|c|c|c|c|c|c|c|c|c|c|c|c|c|}
\hline \multirow{3}{*}{\multicolumn{2}{|c|}{ Model Name }} & \multirow[b]{3}{*}{ Dim. } & \multicolumn{7}{|c|}{ Processes and Approaches } & \multirow[b]{3}{*}{ Activity models } & \multirow[b]{3}{*}{$\begin{array}{c}\text { Sorption } \\
\text { models }\end{array}$} & \multirow[b]{3}{*}{$\begin{array}{l}\text { Solver } \\
\text { Appr. }\end{array}$} & \multirow[b]{3}{*}{$\begin{array}{l}\text { Num. } \\
\text { Meth. }\end{array}$} & \multirow[b]{3}{*}{ Notes } \\
\hline & & & \multirow[b]{2}{*}{ Flow } & \multirow[b]{2}{*}{$\begin{array}{c}\text { Ther- } \\
\text { mal }\end{array}$} & \multirow[b]{2}{*}{$\begin{array}{l}\text { Chem. } \\
\text { Tran- } \\
\text { sport } \\
\end{array}$} & \multicolumn{4}{|c|}{ Reactions } & & & & & \\
\hline & & & & & & $\begin{array}{l}\text { Aq. } \\
\text { Com- } \\
\text { plex }\end{array}$ & Sorp & $\mathbf{P} / \mathbf{D}$ & Bio & & & & & \\
\hline \multirow{3}{*}{$\begin{array}{l}\text { Other codes } \\
\text { utilizing } \\
\text { PHREEQC } \\
\text { for chemistry }\end{array}$} & PHREEQC & $1 \mathrm{D}$ & SF & Yes* & $\mathrm{MC}$ & $\mathrm{EK}^{\mathrm{c}}$ & EK & EK & $\begin{array}{l}\text { Yes } \\
(\mathrm{KI} ?)\end{array}$ & $\begin{array}{l}\text { Davies, extended } \\
\text { Debye-Huckel, } \\
\text { WATEQ Debye- } \\
\text { Huckel, Pitzer, } \\
\text { SIT }\end{array}$ & $\begin{array}{l}\text { DM, NEM, } \\
\text { CDM }\end{array}$ & SIA & FD & $\begin{array}{l}\text { * Incorporates the effects of heat on } \\
\text { transport (reaction kinetics, diffusion and } \\
\text { dispersion), but not on flow (e.g., no } \\
\text { convection). }\end{array}$ \\
\hline & PHAST & $3 \mathrm{D}$ & Yes & No & $\mathrm{MC}$ & $\mathrm{EK}^{\mathrm{c}}$ & EK & EK & $\begin{array}{l}\text { Yes } \\
\text { (KI?) }\end{array}$ & $\begin{array}{c}\text { Davies, extended } \\
\text { Debye-Huckel, } \\
\text { WATEQ Debye- } \\
\text { Huckel, Pitzer, } \\
\text { SIT } \\
\end{array}$ & $\begin{array}{l}\text { DM, NEM, } \\
\text { CDM }\end{array}$ & SNIA & FD & $\begin{array}{l}\text { Based on PHREEQC V.2 and HST3D. } \\
\text { Updated regularly. Parallel version } \\
\text { available. }\end{array}$ \\
\hline & $\begin{array}{l}\text { HP1 } \\
\text { (SCK-CEN) }\end{array}$ & $1 \mathrm{D}$ & VF & Yes* & $\mathrm{MC}$ & $\mathrm{EK}^{\mathrm{c}}$ & EK & EK & No & $\begin{array}{l}\text { Davies, extended } \\
\text { Debye-Huckel, } \\
\text { WATEQ Debye- }^{\text {Huckel }} \\
\end{array}$ & DM, NEM & SNIA & $\mathrm{FE}$ & $\begin{array}{l}\text { Couples HYDRUS-1D and PHREEQC V.2. } \\
\text { * Incorporates the effects of heat on } \\
\text { transport (reactions, diffision and } \\
\text { dispersion), but not on flow (e.g., no } \\
\text { convection). }\end{array}$ \\
\hline \multirow{3}{*}{$\begin{array}{l}\text { Codes } \\
\text { utilizing } \\
\text { CHESS for } \\
\text { chemistry }\end{array}$} & $\begin{array}{l}\text { ALLIANCE } \\
\text { (CAST3M; } \\
\text { CASTEM) } \\
\text { (CEA) } \\
\end{array}$ & $\begin{array}{l}1 \mathrm{D} / \\
2 \mathrm{D} / \\
3 \mathrm{D}\end{array}$ & $?$ & Yes & $\mathrm{MC}$ & EQ & EK & EK & No & $\begin{array}{c}\text { Debye Huckel, } \\
\text { extended Debye } \\
\text { Huckel (B-dot), } \\
\text { Davies } \\
\end{array}$ & $\begin{array}{l}\text { CCM, } \\
\text { DLM, } \\
\text { NEM }\end{array}$ & SIA & $\begin{array}{l}\text { MFE, } \\
\text { FV }\end{array}$ & $\begin{array}{l}\text { Developed by the CEA, combines } \\
\text { CAST3M (hydromechanical code), with } \\
\text { CHESS (chemistry). }\end{array}$ \\
\hline & $\begin{array}{l}\text { CHEMTRAP } \\
\text { (Lucille et al. } \\
\text { 2000) }\end{array}$ & $\begin{array}{l}2 \mathrm{D} / \\
3 \mathrm{D}\end{array}$ & VF & No & $\mathrm{MC}$ & EQ & EK & EK & No & $\begin{array}{l}\text { Debye Huckel, } \\
\text { extended Debye } \\
\text { Huckel (B-dot), } \\
\text { Davies } \\
\end{array}$ & $\begin{array}{l}\text { CCM, } \\
\text { DLM, } \\
\text { NEM }\end{array}$ & SIA & $\mathrm{FE}$ & $\begin{array}{l}\text { Based on coupling of SUBIEF (transport) } \\
\text { and CHESS (chemistry). }\end{array}$ \\
\hline & $\begin{array}{l}\text { HYTEC } \\
\text { (van der Lee } \\
\text { et al. 2002; } \\
\text { van der Lee } \\
\text { et al. 2003) }\end{array}$ & $\begin{array}{l}1 \mathrm{D} / \\
2 \mathrm{D} / \\
3 \mathrm{D}\end{array}$ & VF & Yes & $\mathrm{MC}$ & EQ & $\begin{array}{c}\mathrm{EK} \\
(\mathrm{EQ} ?)\end{array}$ & EK & No & $\begin{array}{l}\text { Debye Huckel, } \\
\text { extended Debye } \\
\text { Huckel (B-dot), } \\
\text { Davies }\end{array}$ & $\begin{array}{l}\text { CCM, } \\
\text { DLM, } \\
\text { NEM, IC }\end{array}$ & SIA & $\begin{array}{l}\mathrm{FD} \\
\mathrm{FE} \\
\mathrm{FV}\end{array}$ & $\begin{array}{l}\text { Modular code, based on the chemical } \\
\text { speciation code CHESS, coupled with one } \\
\text { of three flow and transport codes: RT1D } \\
\text { (1D, FD), METIS (2D/3D, FE), and R2D2 } \\
\text { (2D, FV). Includes colloidal transport. } \\
\text { Parallel version available. }\end{array}$ \\
\hline
\end{tabular}


Table A-1. Reactive Transport Codes with THC Capabilities Potentially Relevant to the Waste IPSC

\begin{tabular}{|c|c|c|c|c|c|c|c|c|c|c|c|c|c|}
\hline \multirow[b]{3}{*}{ Model Name } & \multirow[b]{3}{*}{ Dim. } & \multicolumn{7}{|c|}{ Processes and Approaches } & \multirow[b]{3}{*}{ Activity models } & \multirow[b]{3}{*}{$\begin{array}{c}\text { Sorption } \\
\text { models }\end{array}$} & \multirow[b]{3}{*}{$\begin{array}{l}\text { Solver } \\
\text { Appr. }\end{array}$} & \multirow[b]{3}{*}{$\begin{array}{l}\text { Num. } \\
\text { Meth. }\end{array}$} & \multirow[b]{3}{*}{ Notes } \\
\hline & & \multirow[b]{2}{*}{ Flow } & \multirow[b]{2}{*}{$\begin{array}{l}\text { Ther- } \\
\text { mal }\end{array}$} & \multirow[b]{2}{*}{$\begin{array}{l}\text { Chem. } \\
\text { Tran- } \\
\text { sport } \\
\end{array}$} & \multicolumn{4}{|c|}{ Reactions } & & & & & \\
\hline & & & & & $\begin{array}{l}\text { Aq. } \\
\text { Com- } \\
\text { plex }\end{array}$ & Sorp & $\mathbf{P} / \mathbf{D}$ & Bio & & & & & \\
\hline CRUNCH & $\begin{array}{l}2 \mathrm{D} / \\
3 \mathrm{D}\end{array}$ & VF & Yes & MC & EQ & EQ & KI & Yes & $\begin{array}{l}\text { extended Debye- } \\
\text { Huckel }\end{array}$ & $\begin{array}{l}\text { DLM, } \\
\text { NEM, IE }\end{array}$ & $\begin{array}{l}\text { DSA, } \\
\text { SIA }\end{array}$ & IFV & $\begin{array}{l}\text { Based on codes OS3D and GIMRT. } \\
\text { Implements radioactive decay chains. } \\
\text { OS3D simulations are up to 3D and } \\
\text { implement SIA; GIMRT simulations are up } \\
\text { to 2D, and implement DSA (global } \\
\text { implicit). Restricted to orthogonal grids. }\end{array}$ \\
\hline $\begin{array}{l}\text { FEHM } \\
\text { (LANL) }\end{array}$ & $\begin{array}{l}1 \mathrm{D} / \\
2 \mathrm{D} / \\
3 \mathrm{D}\end{array}$ & $\begin{array}{l}\text { MF, } \\
\text { VF }\end{array}$ & Yes & $\mathrm{MC}^{*}$ & $\mathrm{EK}^{*}$ & Emp. & KI & Yes $^{\mathrm{d}}$ & $\begin{array}{l}\text { All activities } \\
\text { assumed to be } \\
\text { unity? }\end{array}$ & $\begin{array}{l}\text { LI and } \\
\text { NLI, with } \\
\text { kinetics }\end{array}$ & $?$ & CVFE & $\begin{array}{l}\text { Uses particle tracking to model transport. } \\
\text { *Up to } 10 \text { solutes with chemical reactions } \\
\text { between each. Reactions can be kinetic } \\
\text { (e.g., biodegradation). }\end{array}$ \\
\hline $\begin{array}{l}\text { FEMWATER } \\
\text { (WES) }\end{array}$ & $3 \mathrm{D}$ & $\mathrm{VF}$ & No & $\mathrm{SC}$ & No & Emp & No & KI & N/A & LI, NLI & SIA & $\begin{array}{c}\text { FE/ } \\
\text { FE-LE }\end{array}$ & \\
\hline $\begin{array}{l}\text { FRACHEM } \\
\text { (CGR) }\end{array}$ & $3 \mathrm{D}$ & $\mathrm{SF}$ & Yes & $\mathrm{MC}$ & EQ & No & EK & No & Pitzer & N/A & SNIA & $\mathrm{FE}$ & $\begin{array}{l}\text { Based on CHEMTOUGH2 (chemistry) and } \\
\text { FRACTure (flow in fractured, porous rock) }\end{array}$ \\
\hline $\begin{array}{l}\text { GeoSysBRNS } \\
\text { (Centler et al. 2010) }\end{array}$ & $\begin{array}{l}1 \mathrm{D} / \\
2 \mathrm{D} / \\
3 \mathrm{D}\end{array}$ & $\begin{array}{l}\text { MF, } \\
\text { VF }\end{array}$ & Yes & $\mathrm{MC}$ & EK? & $\mathrm{EK} ?$ & EK & EK & $?$ & $?$ & SNIA & $\mathrm{FE}$ & $\begin{array}{l}\text { Couples Biogeochemical Reaction Network } \\
\text { Simulator (BRNS) with GeoSys, a THM } \\
\text { flow and transport simulator. Current } \\
\text { version does not account for changes in } \\
\text { porosity due to mineral prrecipitation. } \\
\text { Parallel version available. }\end{array}$ \\
\hline GWB professional & $\begin{array}{l}1 \mathrm{D} / \\
2 \mathrm{D}\end{array}$ & $\mathrm{No}^{*}$ & Yes & $\mathrm{MC}$ & EK & $?$ & EK & $?$ & $\begin{array}{l}\text { Debye Huckel, } \\
\text { Pitzer }\end{array}$ & $?$ & $?$ & $?$ & $\begin{array}{l}\text { *Flow fields imported as a table or from } \\
\text { MODFLOW }\end{array}$ \\
\hline HYDROGEOCHEM 4.1/5.1 & $\begin{array}{l}2 \mathrm{D} / \\
3 \mathrm{D}\end{array}$ & $\mathrm{VF}$ & Yes & $\mathrm{MC}$ & EK & EK & EK & EK & $\begin{array}{l}\text { Davies equation, } \\
\quad \text { and? }\end{array}$ & $\begin{array}{l}\text { CCM, } \\
\text { DLM, } \\
\text { TLM }\end{array}$ & $\begin{array}{l}\text { DSA, } \\
\text { SIA }\end{array}$ & $\begin{array}{l}\text { FE, } \\
\text { FE-LE }\end{array}$ & $\begin{array}{l}\text { Version } 4.1 \text { is } 2 \mathrm{D} \text {; version } 5.1 \text { is } 3 \mathrm{D} \text {. These } \\
\text { versions of HYDROGEOCHEM appear to } \\
\text { incorporate all capabilities of earlier } \\
\text { versions, as well as of of the codes } \\
\text { developed in parallel (LEHGC, } \\
\text { HYDROBIOGEOCHEM and HGBC123). } \\
\text { Note that (1) applications are limited to } \\
\text { single fluid phase flows and (2) dual- } \\
\text { porosity media cannot be effectively dealt } \\
\text { with. }\end{array}$ \\
\hline HYDRUS (2D/3D) & $\begin{array}{l}2 \mathrm{D} / \\
3 \mathrm{D}\end{array}$ & $\mathrm{VF}$ & Yes & MC & No* & $\begin{array}{l}\text { Emp- } \\
\text { KI }\end{array}$ & No & Yes $^{\mathrm{d}}$ & N/A & $\begin{array}{l}\text { EI; LI and } \\
\text { NLI; uses } \\
\text { two-site } \\
\text { sorption } \\
\text { (fast-slow) } \\
\text { for } \\
\text { sorption } \\
\text { kinetics }\end{array}$ & SNIA & $\mathrm{FE}$ & Includes colloidal transport. \\
\hline
\end{tabular}


Table A-1. Reactive Transport Codes with THC Capabilities Potentially Relevant to the Waste IPSC

\begin{tabular}{|c|c|c|c|c|c|c|c|c|c|c|c|c|c|}
\hline \multirow[b]{3}{*}{ Model Name } & \multirow[b]{3}{*}{ Dim. } & \multicolumn{7}{|c|}{ Processes and Approaches } & \multirow[b]{3}{*}{ Activity models } & \multirow[b]{3}{*}{$\begin{array}{c}\text { Sorption } \\
\text { models }\end{array}$} & \multirow[b]{3}{*}{$\begin{array}{l}\text { Solver } \\
\text { Appr. }\end{array}$} & \multirow[b]{3}{*}{$\begin{array}{l}\text { Num. } \\
\text { Meth. }\end{array}$} & \multirow[b]{3}{*}{ Notes } \\
\hline & & \multirow[b]{2}{*}{ Flow } & \multirow[b]{2}{*}{$\begin{array}{c}\text { Ther- } \\
\text { mal }\end{array}$} & \multirow{2}{*}{$\begin{array}{l}\text { Chem. } \\
\text { Tran- } \\
\text { sport }\end{array}$} & \multicolumn{4}{|c|}{ Reactions } & & & & & \\
\hline & & & & & $\begin{array}{l}\text { Com- } \\
\text { plex }\end{array}$ & Sorp & $\mathbf{P} / \mathbf{D}$ & Bio & & & & & \\
\hline $\begin{array}{l}\text { MCOTAC } \\
\text { (PSI) }\end{array}$ & $\begin{array}{l}1 \mathrm{D} / \\
2 \mathrm{D}\end{array}$ & $?$ & $?$ & $\mathrm{MC}$ & EQ & $\begin{array}{l}\text { Emp. } \\
+?\end{array}$ & EK & No & $?$ & $\begin{array}{c}\mathrm{LI} \\
\mathrm{SCM}(?)\end{array}$ & SNIA & FD & Does not include matrix diffusion. \\
\hline $\begin{array}{r}\text { MIN3P } \\
(\text { UBC) }\end{array}$ & $\begin{array}{l}1 \mathrm{D} / \\
2 \mathrm{D} / \\
3 \mathrm{D}\end{array}$ & $\mathrm{VF}$ & No & $\mathrm{MC}$ & EK & EQ & KI & Yes $^{\mathrm{d}}$ & $\begin{array}{c}\text { extended Debye } \\
\text { Huckel, Davies, } \\
\text { Pitzer } \\
\end{array}$ & NEM, IE & DSA & FV & \\
\hline $\begin{array}{l}\text { MULTIFLO } \\
\text { (SWRI-CNWRA) }\end{array}$ & $\begin{array}{l}1 \mathrm{D} / \\
2 \mathrm{D} / \\
3 \mathrm{D}\end{array}$ & MF & Yes & $\mathrm{MC}$ & EK & Yes? & KI & No? & $?$ & $?$ & $?$ & IFD? & \\
\hline $\begin{array}{l}\text { NUFT-C } \\
\text { ( LLNL) }\end{array}$ & $\begin{array}{l}1 \mathrm{D} / \\
2 \mathrm{D} / \\
3 \mathrm{D}\end{array}$ & $\begin{array}{l}\text { MF, } \\
\text { VF }\end{array}$ & Yes & $\mathrm{MC}$ & EQ & $?$ & KI & No & $\begin{array}{l}\text { extended Debye- } \\
\text { Huckel (B-dot) }\end{array}$ & $?$ & SIA & IFD & Massively parallel. \\
\hline $\begin{array}{l}\text { POLLUTRANS } \\
\text { (Kuechler and Noack 2002) }\end{array}$ & $1 \mathrm{D}$ & $\mathrm{VF}$ & No & $\mathrm{MC}$ & EQ & No & KI & No & Davies equation & N/A & SIA & $\begin{array}{l}\text { IFD } \\
(\mathrm{FV})\end{array}$ & $\begin{array}{l}\text { Calculates transport of water and pollutants } \\
\text { downward through the unsaturated zone. }\end{array}$ \\
\hline $\begin{array}{l}\text { RETRASO-CODEBRIGHT } \\
\text { (Saaltink et al. 2005) }\end{array}$ & $\begin{array}{l}1 \mathrm{D} / \\
2 \mathrm{D} / \\
3 \mathrm{D}\end{array}$ & MF & Yes & $\mathrm{MC}$ & EQ & EQ & EK & No & $\begin{array}{l}\text { extended Debye- } \\
\text { Huckel (B-dot) }\end{array}$ & $\begin{array}{l}\text { CCM, DM, } \\
\text { TM, IE }\end{array}$ & DSA & $\mathrm{FE}$ & $\begin{array}{l}\text { Formed by combining of RETRASO } \\
\text { (reactive transport) with CODEBRIGHT } \\
\text { (multiphase THM--but the mechanical part } \\
\text { was not implemented in RETRASO- } \\
\text { CODEBRIGHT)--these codes were not } \\
\text { coupled, but combined (hence the DSA } \\
\text { solver). }\end{array}$ \\
\hline SMART & $\begin{array}{l}1 \mathrm{D} / \\
2 \mathrm{D} / \\
3 \mathrm{D}\end{array}$ & $\mathrm{VF}$ & No & $\mathrm{SC}$ & No? & Emp & No & Yes $^{\mathrm{d}}$ & N/A? & $\begin{array}{l}\text { LI, NLI, } \\
\text { and uses } \\
\text { intraparticl } \\
\text { e diffusion } \\
\text { to capture } \\
\text { kinetics. }\end{array}$ & $?$ & $?$ & $\begin{array}{l}\text { Streamtube Model for Advective and } \\
\text { Reactive Transport. Commonly used for } \\
\text { organic transport through soils. Not truly } \\
\text { multidimensional, as it converts everything } \\
\text { to 1D streamtubes. }\end{array}$ \\
\hline $\begin{array}{l}\text { STORM } \\
\text { (PNNL) }\end{array}$ & $3 \mathrm{D}$ & MF & Yes & $\mathrm{MC}$ & EK & Emp. & $\mathrm{KI}$ & $\mathrm{Yes}^{\mathrm{d}}$ & $\begin{array}{l}\text { extended Debye } \\
\text { Huckel (B-dot) }\end{array}$ & LI & SIA & FD & $\begin{array}{l}\text { Parallel version available. Implements } \\
\text { radioactive decay. }\end{array}$ \\
\hline $\begin{array}{l}\text { TOUGHREACT } \\
\text { (LBNL) }\end{array}$ & $\begin{array}{l}1 \mathrm{D} / \\
2 \mathrm{D} / \\
3 \mathrm{D}\end{array}$ & MF & Yes & $\mathrm{MC}$ & $\begin{array}{c}\mathrm{EQ} \\
\left(\mathrm{EK}^{*}\right)\end{array}$ & $\begin{array}{l}\text { No } \\
\text { (Emp- } \\
\left.\mathrm{K}^{*}\right)\end{array}$ & EK & $\begin{array}{l}\text { No } \\
\text { (Yes*) }\end{array}$ & $\begin{array}{l}\text { extended Debye- } \\
\text { Huckel (Pitzer in } \\
\text { unreleased } \\
\text { version) }\end{array}$ & N/A & $\begin{array}{l}\text { SIA/ } \\
\text { SNIA }\end{array}$ & $\begin{array}{l}\text { IFDM } \\
(\mathrm{FV})\end{array}$ & $\begin{array}{l}\text { * Xu(2006) created a version of } \\
\text { TOUGHREACT with reaction kinetics for } \\
\text { aqueous species, and kinetic biodegradation } \\
\text { and sorption }\left(\mathrm{K}_{\mathrm{d}} \text {-based). }\right.\end{array}$ \\
\hline $\begin{array}{l}\text { STOMP } \\
\text { (PNNL) } \\
\text { (White and McGrail 2005) }\end{array}$ & $3 \mathrm{D}$ & $\mathrm{VF}^{*}$ & $\mathrm{No}^{*}$ & $\mathrm{MC}$ & EK & Emp. & KI & $\mathrm{KI}$ & $\begin{array}{l}\text { Davies, extended } \\
\text { Debye Huckel } \\
\text { (B-dot), Pitzer }\end{array}$ & $\begin{array}{l}\text { LI with } \\
\text { kinetics }\end{array}$ & SNIA & FD & $\begin{array}{l}\text { STOMP represents a series of modules that } \\
\text { can be coupled together. Some modules are } \\
\text { available in parallel versions. Biological } \\
\text { reactions are incorporated through use of a } \\
\text { preprocessor (BIOGEOCHEM). *Some } \\
\text { versions of STOMP do multiphase, non- } \\
\text { isothermal flow, but the version coupled } \\
\text { with the chemical module does not. }\end{array}$ \\
\hline
\end{tabular}


Table A-1. Reactive Transport Codes with THC Capabilities Potentially Relevant to the Waste IPSC

\begin{tabular}{|c|c|c|c|c|c|c|c|c|c|c|c|c|c|}
\hline \multirow[b]{3}{*}{ Model Name } & \multirow[b]{3}{*}{ Dim. } & \multicolumn{7}{|c|}{ Processes and Approaches } & \multirow[b]{3}{*}{ Activity models } & \multirow[b]{3}{*}{$\begin{array}{c}\text { Sorption } \\
\text { models }\end{array}$} & \multirow[b]{3}{*}{$\begin{array}{l}\text { Solver } \\
\text { Appr. }\end{array}$} & \multirow[b]{3}{*}{$\begin{array}{l}\text { Num. } \\
\text { Meth. }\end{array}$} & \multirow[b]{3}{*}{ Notes } \\
\hline & & \multirow[b]{2}{*}{ Flow } & \multirow[b]{2}{*}{$\begin{array}{c}\text { Ther- } \\
\text { mal }\end{array}$} & \multirow{2}{*}{$\begin{array}{l}\text { Chem. } \\
\text { Tran- } \\
\text { sport }\end{array}$} & \multicolumn{4}{|c|}{ Reactions } & & & & & \\
\hline & & & & & $\begin{array}{c}\text { Com- } \\
\text { plex }\end{array}$ & Sorp & $\mathbf{P} / \mathbf{D}$ & Bio & & & & & \\
\hline UTCHEM & $3 \mathrm{D}$ & MF & No & $\mathrm{MC}$ & EQ & Emp. & $\begin{array}{c}\mathrm{EQ} / \mathrm{KI} \\
*\end{array}$ & Yes $^{d}$ & $\begin{array}{l}\text { All activities } \\
\text { assumed to be } \\
\text { unity }\end{array}$ & LI, NLI & $?$ & FD & $\begin{array}{l}\text { Chemical flooding simulator for oilfield } \\
\text { reservoirs and for bioremediation of } \\
\text { organics in aquifers. Chemical database is } \\
\text { limited to reactions important to these } \\
\text { processes. } \\
\text { * Precipitation/dissolution of minerals is } \\
\text { handled as an equilibrium process, but gels } \\
\text { are treated as a kinetic process. Organics } \\
\text { dissolve kinetically. }\end{array}$ \\
\hline $\begin{array}{l}\text { 3FLO } \\
\text { (ITASCA) }\end{array}$ & $3 \mathrm{D}$ & VF & No? & $\mathrm{MC}$ & Yes? & Yes? & $\mathrm{KI}$ ? & No? & $?$ & $?$ & $?$ & $\begin{array}{l}\text { FE, } \\
\text { MFE }\end{array}$ & $\begin{array}{l}\text { Simulates flow in fracture networks as a 3D } \\
\text { network of } 1 \mathrm{D} \text { pipes or channels. Simulates } \\
\text { transport by the random walk (particle } \\
\text { tracking) method. }\end{array}$ \\
\hline
\end{tabular}

a. Members of the CORE family can only do 3-D for axially symmetric cases.

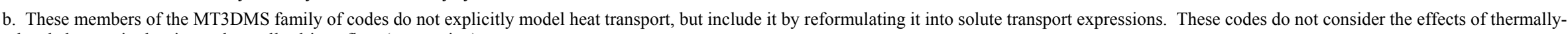

related changes in density or thermally-driven flow (convection).

c. Codes implementing PHREEQC as the chemistry solver can apply kinetics to aqueous species reactions, but only if the species are redefined in the database as solution master species.

d. These codes implement biodegradation only, through kinetic reactions

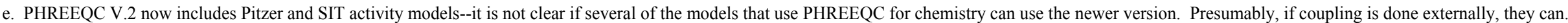

\section{Legend/Acronyms for Table A-1}

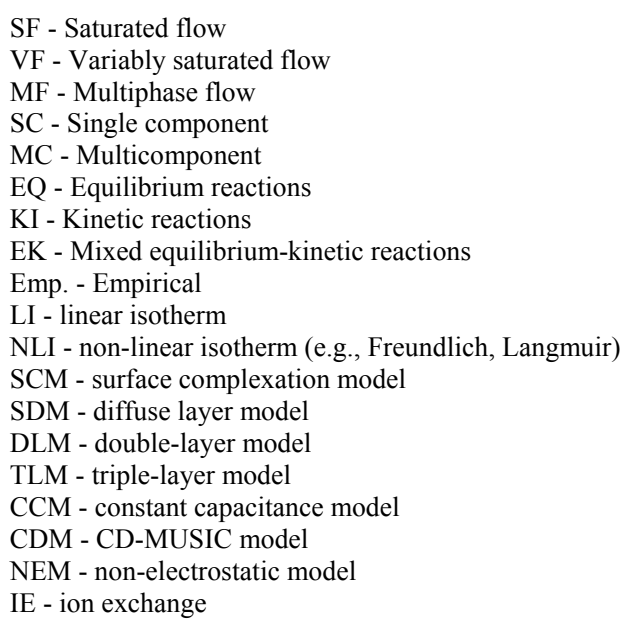

SIA - Sequential iterative approach

SNIA - Sequential noniterative approach

SPIA - Sequential partly interative approach

DSA - Direct substitution approach (same as Global implicit method, GIM)

FE - Finite element

FV - Finite volume

FD - Finite difference

IFD - Integrated finite difference (equivalent to finite volume)

MFE - Mixed-hybrid finite elements

CVFE - Control volume finite element

LE - Lagrangian-Eulerian 


\section{DISTRIBUTION}

$\begin{array}{llll}1 & \text { MS0370 } & \text { Randall Summers } & 1433 \\ 1 & \text { MS0372 } & \text { J. Guadalupe Argüello } & 1525 \\ 1 & \text { MS0382 } & \text { H. Carter Edwards } & 1433 \\ 1 & \text { MS0751 } & \text { Thomas Dewers } & 6375 \\ 1 & \text { MS0754 } & \text { Louise Criscenti } & 6376 \\ 1 & \text { MS0779 } & \text { Yifeng Wang } & 6772 \\ 1 & \text { MS1138 } & \text { Julie Bouchard } & 6374 \\ 1 & \text { MS1322 } & \text { Peter Schultz } & 1435 \\ 1 & \text { MS1369 } & \text { Geoff Freeze } & 6783 \\ 1 & \text { MS1399 } & \text { David Sassani } & 6782 \\ & & & \\ 1 & \text { MS0899 } & \text { Technical Library } & 9536 \text { (electronic copy) }\end{array}$


Distribution-2 
(1) Sandia National Laboratories 